\title{
Properties of Generalized Univariate Hypergeometric Functions
}

\author{
F. J. van de Bult ${ }^{1}$, E. M. Rains ${ }^{2, \star}$, J. V. Stokman ${ }^{1}$ \\ ${ }^{1} \mathrm{KdV}$ Institute for Mathematics, Universiteit van Amsterdam, Plantage Muidergracht 24, 1018 TV \\ Amsterdam, The Netherlands. E-mail: fjvdbult@science.uva.nl; jstokman@science.uva.nl \\ 2 Department of Mathematics, University of California, Davis, USA. E-mail: rains@math.ucdavis.edu
}

Received: 4 August 2006 / Accepted: 4 January 2007

Published online: 24 July 2007 - (C) Springer-Verlag 2007

\begin{abstract}
Based on Spiridonov's analysis of elliptic generalizations of the Gauss hypergeometric function, we develop a common framework for 7-parameter families of generalized elliptic, hyperbolic and trigonometric univariate hypergeometric functions. In each case we derive the symmetries of the generalized hypergeometric function under the Weyl group of type $E_{7}$ (elliptic, hyperbolic) and of type $E_{6}$ (trigonometric) using the appropriate versions of the Nassrallah-Rahman beta integral, and we derive contiguous relations using fundamental addition formulas for theta and sine functions. The top level degenerations of the hyperbolic and trigonometric hypergeometric functions are identified with Ruijsenaars' relativistic hypergeometric function and the Askey-Wilson function, respectively. We show that the degeneration process yields various new and known identities for hyperbolic and trigonometric special functions. We also describe an intimate connection between the hyperbolic and trigonometric theory, which yields an expression of the hyperbolic hypergeometric function as an explicit bilinear sum in trigonometric hypergeometric functions.
\end{abstract}

\section{Introduction}

The Gauss hypergeometric function, one of the cornerstones in the theory of classical univariate special functions, has been generalized in various fundamental directions. A theory on multivariate root system analogues of the Gauss hypergeometric function, due to Heckman and Opdam, has emerged, forming the basic tools to solve trigonometric and hyperbolic quantum many particle systems of Calogero-Moser type and generalizing the Harish-Chandra theory of spherical functions on Riemannian symmetric spaces (see [8] and references therein). A further important development has been the generalization to $q$-special functions, leading to the theory of Macdonald polynomials [16],

\footnotetext{
* Current address: Department of Mathematics, California Institute of Technology, Davis, USA. E-mail: rains@caltech.edu
} 
which play a fundamental role in the theory of relativistic analogues of the trigonometric quantum Calogero-Moser systems (see e.g. [25]) and in harmonic analysis on quantum compact symmetric spaces (see e.g. $[18,14]$ ). In this paper, we focus on far-reaching generalizations of the Gauss hypergeometric function within the classes of elliptic, hyperbolic and trigonometric univariate special functions.

Inspired by results on integrable systems, Ruijsenaars [24] defined gamma functions of rational, trigonometric, hyperbolic and elliptic type. Correspondingly there are four types of special function theories, with the rational (resp. trigonometric) theory being the standard theory on hypergeometric (resp. $q$-hypergeometric) special functions, while the hyperbolic theory is well suited to deal with unimodular base $q$. The theory of elliptic special functions, initiated by Frenkel and Turaev in [4], is currently in rapid development. The starting point of our analysis is the definition of the various generalized hypergeometric functions as an explicit hypergeometric integral of elliptic, hyperbolic and trigonometric type depending on seven auxiliary parameters (besides the bases). The elliptic and hyperbolic analogue of the hypergeometric function are due to Spiridonov [33], while the trigonometric analogue of the hypergeometric function is essentially an integral representation of the function $\Phi$ introduced and studied extensively by Gupta and Masson in [7]. Under a suitable parameter discretization, the three classes of generalized hypergeometric functions reduce to Rahman's [20] (trigonometric), Spiridonov's [33] (hyperbolic), and Spiridonov's and Zhedanov's [35, 33] (elliptic) families of biorthogonal rational functions.

Spiridonov [33] gave an elementary derivation of the symmetry of the elliptic hypergeometric function with respect to a twisted action of the Weyl group of type $E_{7}$ on the parameters using the elliptic analogue [31] of the Nassrallah-Rahman [17] beta integral. In this paper we follow the same approach to establish the $E_{6}$-symmetry (respectively $E_{7}$-symmetry) of the trigonometric (respectively hyperbolic) hypergeometric function, using now the Nassrallah-Rahman beta integral (respectively its hyperbolic analogue from [37]). The $E_{6}$-symmetry of $\Phi$ has recently been established in [15] by different methods. Spiridonov [33] also gave elementary derivations of contiguous relations for the elliptic hypergeometric function using the fundamental addition formula for theta functions (see (3.6)), entailing a natural elliptic analogue of the Gauss hypergeometric differential equation. Following the same approach we establish contiguous relations and generalized Gauss hypergeometric equations for the hyperbolic and trigonometric hypergeometric function. For $\Phi$ it again leads to simple proofs of various results from [7].

Although the elliptic hypergeometric function is the most general amongst the generalized hypergeometric functions under consideration (rigorous limits between the different classes of special functions have been obtained in the recent paper [23] of the second author), it is also the most rigid in its class, in the sense that it does not admit natural degenerations within the class of elliptic special functions itself (there is no preferred limit point on an elliptic curve). On the other hand, for the hyperbolic and trigonometric hypergeometric functions various interesting degenerations within their classes are possible, as we point out in this paper. It leads to many nontrivial identities and results, some of which are new and some are well known. In any case, it provides new insight in identities, e.g. as being natural consequences of symmetry breaking in the degeneration process, and it places many identities and classes of univariate special functions in a larger framework. For instance, viewing the trigonometric hypergeometric function as a degeneration of the elliptic hypergeometric function, we show that the breaking of symmetry (from $E_{7}$ to $E_{6}$ ) leads to a second important integral representation of $\Phi$. 
Moreover we show that Ruijsenaars' [26] relativistic analogue $R$ of the hypergeometric function is a degeneration of the hyperbolic hypergeometric function, and that the $D_{4}$-symmetry [28] of $R$ and the four Askey-Wilson second-order difference equations [26] satisfied by $R$ are direct consequences of the $E_{7}$-symmetry and the contiguous relations of the hyperbolic hypergeometric function. Similarly, the Askey-Wilson function [11] is shown to be a degeneration of the trigonometric hypergeometric function. In this paper we aim at deriving the symmetries of (degenerate) hyperbolic and trigonometric hypergeometric functions directly from appropriate hyperbolic and trigonometric beta integral evaluations using the above mentioned techniques of Spiridonov [33]. The rational level, in which case the Wilson function [6] appears as a degeneration, will be discussed in a subsequent paper of the first author.

We hope that the general framework proposed in this paper will shed light on the fundamental, common structures underlying various quantum relativistic Calogero-Moser systems and various quantum noncompact homogeneous spaces. In the present univariate setting, degenerations and specializations of the generalized hypergeometric functions play a key role in solving rank one cases of quantum relativistic integrable CalogeroMoser systems and in harmonic analysis on various quantum $\mathrm{SL}_{2}$ groups. On the elliptic level, the elliptic hypergeometric function provides solutions of particular cases of van Diejen's [2] very general quantum relativistic Calogero-Moser systems of elliptic type (see e.g. [33]), while elliptic biorthogonal rational functions have been identified with matrix coefficients of the elliptic quantum $\mathrm{SL}_{2}$ group in [12]. On the hyperbolic level, the Ruijsenaars' R-function solves the rank one case of a quantum relativistic Calogero-Moser system of hyperbolic type (see [29]) and arises as a matrix coefficient of the modular double of the quantum $\mathrm{SL}_{2}$ group (see [1]). On the trigonometric level, similar results are known for the Askey-Wilson function, which is a degeneration of the trigonometric hypergeometric function (see [11] and [10]). For higher rank only partial results are known, see e.g. [13, 21] (elliptic) and [36] (trigonometric).

The outline of the paper is as follows. In Sect. 2 we discuss the general pattern of symmetry breaking when integrals with $E_{7}$-symmetry are degenerated. In Sect. 3 we introduce Spiridonov's [33] elliptic hypergeometric function. We shortly recall Spiridonov's [33] techniques to derive the $E_{7}$-symmetry and the contiguous relations for the elliptic hypergeometric function. In Sect. 4 these techniques are applied for the hyperbolic hypergeometric function and its top level degenerations. We show that a reparametrization of the top level degeneration of the hyperbolic hypergeometric function is Ruijsenaars' [26] relativistic hypergeometric function $R$. Key properties of $R$, such as a new integral representation, follow from the symmetries and contiguous relations of the hyperbolic hypergeometric function. In Sect. 5 these techniques are considered on the trigonometric level. We link the top level degeneration of the trigonometric hypergeometric function to the Askey-Wilson function. Moreover, we show that the techniques lead to elementary derivations of series representations and three term recurrence relations of the various trigonometric integrals. The trigonometric integrals are contour integrals over indented unit circles in the complex plane, which can be re-expressed as integrals over the real line with indentations by "unfolding" the trigonometric integral. We show that this provides a link with Agarwal type integral representations of basic hypergeometric series (see [5, Chap. 4]). Finally, in Sect. 6 we extend the techniques from [37] to connect the hyperbolic and trigonometric theory. It leads to an explicit expression of the hyperbolic hypergeometric function as a bilinear sum of trigonometric hypergeometric functions. In the top level degeneration, it explicitly relates Ruijsenaars' relativistic hypergeometric function to the Askey-Wilson function. 
1.1. Notation. We denote $\sqrt{ } \cdot$ for the branch of the square root $z \mapsto z^{\frac{1}{2}}$ on $\mathbb{C} \backslash \mathbb{R}_{<0}$ with positive values on $\mathbb{R}_{>0}$.

\section{Weyl Groups and Symmetry Breaking}

The root system of type $E_{7}$ and its parabolic root sub-systems plays an important role in this article. In this section we describe our specific choice of realization of the root systems and Weyl groups, and we explain the general pattern of symmetry breaking which arises from degenerating integrals with Weyl group symmetries.

Degeneration of integrals with Weyl group symmetries in general causes symmetry breaking since the direction of degeneration in parameter space is not invariant under the symmetry group. All degenerations we consider are of the following form. For a basis $\Delta$ of a given irreducible, finite root system $R$ in Euclidean space $(V,\langle\cdot, \cdot\rangle)$ with associated Weyl group $W$ we denote

$$
V^{+}(\Delta)=\{v \in V \mid\langle v, \alpha\rangle \geq 0 \quad \forall \alpha \in \Delta\}
$$

for the associated positive Weyl chamber. We will study integrals $I(u)$ meromorphically depending on a parameter $u \in \mathcal{G}$. The parameter space will be some complex hyperplane $\mathcal{G}$ canonically isomorphic to the complexification $V_{\mathbb{C}}$ of $V$, from which it inherits a $W$-action. The integrals under consideration will be $W$-invariant under an associated twisted $W$-action. We degenerate such integrals by taking limits in parameter space along distinguished directions $v \in V^{+}(\Delta)$. The resulting degenerate integrals will thus inherit symmetries with respect to the isotropy subgroup

$$
W_{v}=\{\sigma \in W \mid \sigma v=v\},
$$

which is a standard parabolic subgroup of $W$ with respect to the given basis $\Delta$, generated by the simple reflections $s_{\alpha}, \alpha \in \Delta \cap v^{\perp}$ (since $v \in V^{+}(\Delta)$ ).

All symmetry groups we will encounter are parabolic subgroups of the Weyl group $W$ of type $E_{8}$. We use in this article the following explicit realization of the root system $R\left(E_{8}\right)$ of type $E_{8}$. Let $\epsilon_{k}$ be the $k^{\text {th }}$ element of the standard orthonormal basis of $V=\mathbb{R}^{8}$, with corresponding scalar product denoted by $\langle\cdot, \cdot\rangle$. We also denote $\langle\cdot, \cdot\rangle$ for its complex bilinear extension to $\mathbb{C}^{8}$. We write $\delta=\frac{1}{2}\left(\epsilon_{1}+\epsilon_{2}+\cdots+\epsilon_{8}\right)$. We realize the root system $R\left(E_{8}\right)$ of type $E_{8}$ in $\mathbb{R}^{8}$ as

$$
R\left(E_{8}\right)=\left\{v=\sum_{j=1}^{8} c_{j} \epsilon_{j}+c \delta \mid\langle v, v\rangle=2, c_{j}, c \in \mathbb{Z} \text { and } \sum_{j=1}^{8} c_{j} \text { even }\right\} .
$$

For later purposes, it is convenient to have explicit notations for the roots in $R\left(E_{8}\right)$. The roots are $\pm \alpha_{j k}^{+}(1 \leq j<k \leq 8), \alpha_{j k}^{-}(1 \leq j \neq k \leq 8), \beta_{j k l m}(1 \leq j<k<l<m \leq 8)$, $\pm \gamma_{j k}(1 \leq j<k \leq 8)$ and $\pm \delta$, where

$$
\begin{aligned}
\alpha_{j k}^{+} & =\epsilon_{j}+\epsilon_{k}, \\
\alpha_{j k}^{-} & =\epsilon_{j}-\epsilon_{k}, \\
\beta_{j k l m} & =\frac{1}{2}\left(\epsilon_{j}+\epsilon_{k}+\epsilon_{l}+\epsilon_{m}-\epsilon_{n}-\epsilon_{p}-\epsilon_{q}-\epsilon_{r}\right), \\
\gamma_{j k} & =\frac{1}{2}\left(-\epsilon_{j}-\epsilon_{k}+\epsilon_{l}+\epsilon_{m}+\epsilon_{n}+\epsilon_{p}+\epsilon_{q}+\epsilon_{r}\right),
\end{aligned}
$$

and with $(j, k, l, m, n, p, q, r)$ a permutation of $(1,2,3,4,5,6,7,8)$. 
The canonical action of the associated Weyl group $W$ on $\mathbb{C}^{8}$ is determined by the reflections $s_{\gamma} u=u-\langle u, \gamma\rangle \gamma$ for $u \in \mathbb{C}^{8}$ and $\gamma \in R\left(E_{8}\right)$. It is convenient to work with two different choices $\bar{\Delta}_{1}, \bar{\Delta}_{2}$ of bases for $R\left(E_{8}\right)$, namely

$$
\begin{aligned}
& \bar{\Delta}_{1}=\left\{\alpha_{76}^{-}, \beta_{1234}, \alpha_{65}^{-}, \alpha_{54}^{-}, \alpha_{43}^{-}, \alpha_{32}^{-}, \alpha_{21}^{-}, \alpha_{18}^{+}\right\}, \\
& \bar{\Delta}_{2}=\left\{\alpha_{23}^{-}, \alpha_{56}^{-}, \alpha_{34}^{-}, \alpha_{45}^{-}, \beta_{5678}, \alpha_{18}^{-}, \alpha_{87}^{-}, \gamma_{18}\right\},
\end{aligned}
$$

with corresponding (affine) Dynkin diagrams

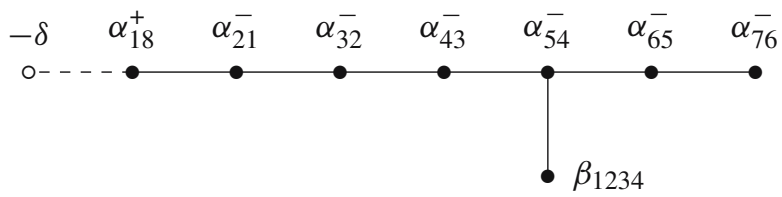

and

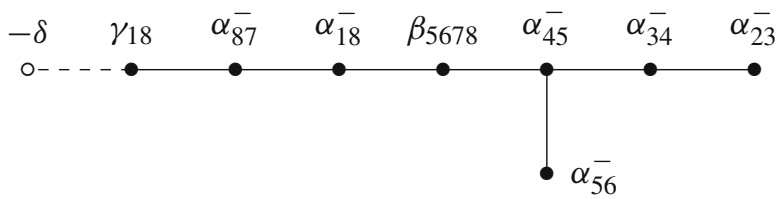

respectively, where the open node corresponds to the simple affine root, which we have labeled by minus the highest root of $R\left(E_{8}\right)$ with respect to the given basis (which in both cases is given by $\delta \in V^{+}\left(\Delta_{j}\right)$ ). The reason for considering two different bases is the following: we will see that degenerating an elliptic hypergeometric integral with $W\left(E_{7}\right)$ symmetry to the trigonometric level in the direction of the basis element $\alpha_{18}^{+} \in \bar{\Delta}_{1}$, respectively the basis element $\gamma_{18} \in \bar{\Delta}_{2}$, leads to two essentially different trigonometric hypergeometric integrals with $W\left(E_{6}\right)$-symmetry. The two integrals can be easily related since they arise as a degeneration of the same elliptic hypergeometric integral. This leads directly to highly nontrivial trigonometric identities, see Sect. 5 for details.

This remark in fact touches on the basic philosophy of this paper: it is the symmetry breaking in the degeneration of hypergeometric integrals which lead to various nontrivial identities. It forms an explanation why there are so many more nontrivial identities on the hyperbolic, trigonometric and rational level when compared to the elliptic level.

Returning to the precise description of the relevant symmetry groups, we will mainly encounter stabilizer subgroups of the isotropy subgroup $W_{-\delta}$. Observe that $W_{-\delta}$ is a standard parabolic subgroup of $W$ with respect to both bases $\bar{\Delta}_{j}$ since $-\delta \in V^{+}\left(\bar{\Delta}_{j}\right)$ $(j=1,2)$, with associated simple reflections $s_{\alpha}, \alpha \in \Delta_{1}:=\bar{\Delta}_{1} \backslash\left\{\alpha_{18}^{+}\right\}$, respectively $s_{\alpha}, \alpha \in \Delta_{2}:=\bar{\Delta}_{2} \backslash\left\{\gamma_{18}\right\}$. Hence $W_{-\delta}$ is isomorphic to the Weyl group of type $E_{7}$, and we accordingly write

$$
W\left(E_{7}\right):=W_{-\delta} .
$$

We realize the corresponding standard parabolic root system $R\left(E_{7}\right) \subset R\left(E_{8}\right)$ as

$$
R\left(E_{7}\right)=R\left(E_{8}\right) \cap \delta^{\perp} \subseteq \delta^{\perp} \subset \mathbb{R}^{8} .
$$

Both $\Delta_{1}$ and $\Delta_{2}$ form a basis of $R\left(E_{7}\right)$, and the associated (affine) Dynkin diagrams are given by

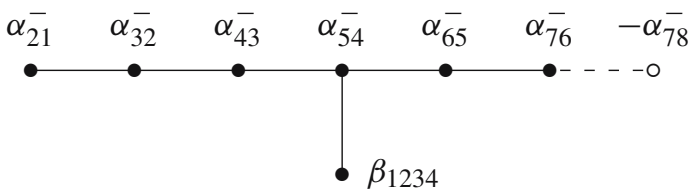


and

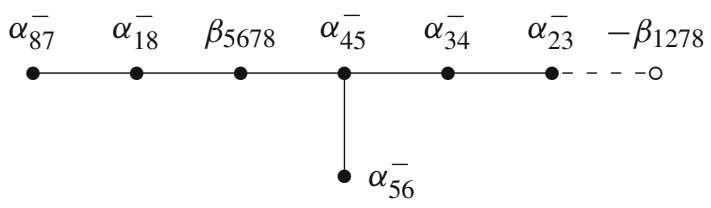

respectively (where we have used that $\alpha_{78}^{-}$, respectively $\beta_{1278}$, is the highest root of $R\left(E_{7}\right)$ with respect to the basis $\Delta_{1}$, respectively $\left.\Delta_{2}\right)$. Note that the root system $R\left(E_{7}\right)$ consists of the roots of the form $\alpha_{j k}^{-}$and $\beta_{j k l m}$.

The top level univariate hypergeometric integrals which we will consider in this article depend meromorphically on a parameter $u \in \mathcal{G}_{c}$ with $\mathcal{G}_{c} \subset V_{\mathbb{C}}=\mathbb{C}^{8}(c \in \mathbb{C})$ the complex hyperplane

$$
\mathcal{G}_{c}=\frac{c}{2} \delta+\delta^{\perp}=\left\{u=\left(u_{1}, u_{2}, \ldots, u_{8}\right) \in \mathbb{C}^{8} \mid \sum_{j=1}^{8} u_{j}=2 c\right\} .
$$

The action on $\mathbb{C}^{8}$ of the isotropy subgroup $W\left(E_{7}\right)=W_{-\delta} \subset W$ preserves the hyperplane $\delta^{\perp}$ and fixes $\delta$, hence it canonically acts on $\mathcal{G}_{c}$. We extend it to an action of the associated affine Weyl group $W_{a}\left(E_{7}\right)$ of $R\left(E_{7}\right)$ as follows. Denote $L$ for the $\left(W\left(E_{7}\right)\right.$ invariant) root lattice $L \subset \delta^{\perp}$ of $R\left(E_{7}\right)$, defined as the $\mathbb{Z}$-span of all $R\left(E_{7}\right)$-roots. The affine Weyl group $W_{a}\left(E_{7}\right)$ is the semi-direct product group $W_{a}\left(E_{7}\right)=W\left(E_{7}\right) \ltimes L$. The action of $W\left(E_{7}\right)$ on $\mathcal{G}_{c}$ can then be extended to an action of the affine Weyl group $W_{a}\left(E_{7}\right)$ depending on an extra parameter $z \in \mathbb{C}$ by letting $\gamma \in L$ act as the shift

$$
\tau_{\gamma}^{z} u=u-z \gamma, \quad u \in \mathcal{G}_{c} .
$$

We suppress the dependence on $z$ whenever its value is implicitly clear from context.

We also use a multiplicative version of the $W\left(E_{7}\right)$-action on $\mathcal{G}_{c}$. Consider the action of the group $C_{2}$ of order two on $\mathbb{C}^{8}$, with the non-unit element of $C_{2}$ acting by multiplication by -1 of each coordinate. We define the parameter space $\mathcal{H}_{c}$ for a parameter $c \in \mathbb{C}^{\times}=\mathbb{C} \backslash\{0\}$ as

$$
\mathcal{H}_{c}=\left\{t=\left(t_{1}, \ldots, t_{8}\right) \in \mathbb{C}^{8} \mid \prod_{j=1}^{8} t_{j}=c^{2}\right\} / C_{2} .
$$

Note that this is well defined because if $t$ satisfies $\prod t_{i}=c^{2}$, then so does $-t$. We sometimes abuse notation by simply writing $t=\left(t_{1}, \ldots, t_{8}\right)$ for the element $\pm t$ in $\mathcal{H}_{c}$ if no confusion can arise.

We view the parameters $t \in \mathcal{H}_{\exp (c)}$ as the exponential parameters associated to $u \in \mathcal{G}_{c}$. Modding out by the action of the 2-group $C_{2}$ allows us to put a $W_{a}\left(E_{7}\right)$-action on $\mathcal{H}_{\exp (c)}$, which is compatible to the $W_{a}\left(E_{7}\right)$-action on $\mathcal{G}_{c}$ as defined above. Concretely, consider the surjective map $\psi_{c}: \mathcal{G}_{c} \rightarrow \mathcal{H}_{\exp (c)}$ defined by

$$
\psi_{c}(u)= \pm\left(\exp \left(u_{1}\right), \ldots, \exp \left(u_{8}\right)\right), \quad u \in \mathcal{G}_{c} .
$$

For $u \in \mathcal{G}_{c}$ we have $\psi_{c}^{-1}\left(\psi_{c}(u)\right)=u+2 \pi i L$, where $L$ is the root lattice of $R\left(E_{7}\right)$ as defined above. Since $L$ is $W\left(E_{7}\right)$-invariant, we can now define the action of $W_{a}\left(E_{7}\right)$ on $\mathcal{H}_{\exp (c)}$ by $\sigma \psi_{c}(u)=\psi_{c}(\sigma u), \sigma \in W_{a}$ (for any auxiliary parameter $z \in \mathbb{C}$ ). 
Regardless of whether we view the action of the affine Weyl group additively or multiplicatively, we will use the abbreviated notations $s_{j k}=s_{\alpha_{j k}^{-}}, w=s_{\beta_{1234}}$ and $\tau_{j k}^{z}=\tau_{\alpha_{j k}^{-}}^{z}$ throughout the article. Note that $s_{j k}(j \neq k)$ acts by interchanging the $j^{\text {th }}$ and $k^{\text {th }}$ coordinate. Furthermore, $W\left(E_{7}\right)$ is generated by the simple reflections $s_{\alpha}\left(\alpha \in \Delta_{1}\right)$, which are the simple permutations $s_{j, j+1}(j=1, \ldots, 6)$ and $w$. The multiplicative action of $w$ on $\mathcal{H}_{c}$ is explicitly given by $w( \pm t)= \pm\left(s t_{1}, s t_{2}, s t_{3}, s t_{4}, s^{-1} t_{5}, s^{-1} t_{6}, s^{-1} t_{7}, s^{-1} t_{8}\right)$, where $s^{2}=c / t_{1} t_{2} t_{3} t_{4}=t_{5} t_{6} t_{7} t_{8} / c$. Finally, note that the longest element $v$ of the Weyl group $W\left(E_{7}\right)$ acts by multiplication with -1 on the root system $R\left(E_{7}\right)$, and hence it acts by $v u=c / 2-u$ on $\mathcal{G}_{c}$ and by $v( \pm t)= \pm\left(c^{\frac{1}{2}} / t_{1}, \ldots, c^{\frac{1}{2}} / t_{8}\right)$ on $\mathcal{H}_{c}$.

\section{The Univariate Elliptic Hypergeometric Function}

3.1. The elliptic gamma function. We will use notations which are consistent with [5]. We fix throughout this section two bases $p, q \in \mathbb{C}$ satisfying $|p|,|q|<1$. The $q$-shifted factorial is defined by

$$
(a ; q)_{\infty}=\prod_{j=0}^{\infty}\left(1-a q^{j}\right) .
$$

We write $\left(a_{1}, \ldots, a_{m} ; q\right)_{\infty}=\prod_{j=1}^{m}\left(a_{j} ; q\right)_{\infty},\left(a z^{ \pm 1} ; q\right)_{\infty}=\left(a z, a z^{-1} ; q\right)_{\infty}$ etc. as shorthand notations for products of $q$-shifted factorials. The renormalized Jacobi thetafunction is defined by

$$
\theta(a ; q)=(a, q / a ; q)_{\infty} .
$$

The elliptic gamma function [24], defined by the infinite product

$$
\Gamma_{e}(z ; p, q)=\prod_{j, k=0}^{\infty} \frac{1-z^{-1} p^{j+1} q^{k+1}}{1-z p^{j} q^{k}},
$$

is a meromorphic function in $z \in \mathbb{C}^{\times}=\mathbb{C} \backslash\{0\}$ which satisfies the difference equation

$$
\Gamma_{e}(q z ; p, q)=\theta(z ; p) \Gamma_{e}(z ; p, q),
$$

satisfies the reflection equation

$$
\Gamma_{e}(z ; p, q)=1 / \Gamma_{e}(p q / z ; p, q),
$$

and is symmetric in $p$ and $q$,

$$
\Gamma_{e}(z ; p, q)=\Gamma_{e}(z ; q, p) .
$$

For products of theta-functions and elliptic gamma functions we use the same shorthand notations as for the $q$-shifted factorial, e.g.

$$
\Gamma_{e}\left(a_{1}, \ldots, a_{m} ; p, q\right)=\prod_{j=1}^{m} \Gamma_{e}\left(a_{j} ; p, q\right) .
$$

In this section we call a sequence of points a downward (respectively upward) sequence of points if it is of the form $a p^{j} q^{k}$ (respectively $a p^{-j} q^{-k}$ ) with $j, k \in \mathbb{Z}_{\geq 0}$ for some $a \in \mathbb{C}$. Observe that the elliptic gamma function $\Gamma_{e}(a z ; p, q)$, considered as a meromorphic function in $z$, has poles at the upward sequence $a^{-1} p^{-j} q^{-k}\left(j, k \in \mathbb{Z}_{\geq 0}\right)$ of points and has zeros at the downward sequence $a^{-1} p^{j+1} q^{k+1}\left(j, k \in \mathbb{Z}_{\geq 0}\right)$ of points. 
3.2. Symmetries of the elliptic hypergeometric function. The fundamental starting point of our investigations is Spiridonov's [31] elliptic analogue of the classical beta integral,

$$
\frac{(q ; q)_{\infty}(p ; p)_{\infty}}{2} \int_{\mathcal{C}} \frac{\prod_{j=1}^{6} \Gamma_{e}\left(t_{j} z^{ \pm 1} ; p, q\right)}{\Gamma_{e}\left(z^{ \pm 2} ; p, q\right)} \frac{d z}{2 \pi i z}=\prod_{1 \leq j<k \leq 6} \Gamma_{e}\left(t_{j} t_{k} ; p, q\right)
$$

for generic parameters $t \in \mathbb{C}^{6}$ satisfying the balancing condition $\prod_{j=1}^{6} t_{j}=p q$, where the contour $\mathcal{C}$ is chosen as a deformation of the positively oriented unit circle $\mathbb{T}$ separating the downward sequences $t_{j} p^{\mathbb{Z}_{\geq 0}} q^{\mathbb{Z}_{\geq 0}}(j=1, \ldots, 6)$ of poles of the integrand from the upward sequences $t_{j}^{-1} p^{\mathbb{Z}_{\leq 0}} q^{\mathbb{Z}_{\leq 0}}(j=1, \ldots, 6)$. Note here that the factor $1 / \Gamma_{e}\left(z^{ \pm 2} ; p, q\right)$ of the integrand is analytic on $\mathbb{C}^{\times}$. Moreover, observe that we can take the positively oriented unit circle $\mathbb{T}$ as contour if the parameters satisfy $\left|t_{j}\right|<1$ $(j=1, \ldots, 6)$. Several elementary proofs of (3.2) are now known, see e.g. [31,32 and 21].

We define the integrand $I_{e}(t ; z)=I_{e}(t ; z ; p, q)$ for the univariate elliptic hypergeometric function as

$$
I_{e}(t ; z ; p, q)=\frac{\prod_{j=1}^{8} \Gamma_{e}\left(t_{j} z^{ \pm 1} ; p, q\right)}{\Gamma_{e}\left(z^{ \pm 2} ; p, q\right)}
$$

where $t=\left(t_{1}, t_{2}, \ldots, t_{8}\right) \in\left(\mathbb{C}^{\times}\right)^{8}$. For parameters $t \in \mathbb{C}^{8}$ with $\prod_{j=1}^{8} t_{j}=p^{2} q^{2}$ and $t_{i} t_{j} \notin p^{\mathbb{Z}_{\leq 0}} q^{\mathbb{Z}_{\leq 0}}$ for $1 \leq i, j \leq 8$ (possibly equal), we can define the elliptic hypergeometric function $S_{e}(t)=S_{e}(t ; p, q)$ by

$$
S_{e}(t ; p, q)=\int_{\mathcal{C}} I_{e}(t ; z ; p, q) \frac{d z}{2 \pi i z},
$$

where the contour $\mathcal{C}$ is a deformation of $\mathbb{T}$ which separates the downward sequences $t_{j} p^{\mathbb{Z}_{\geq 0}} q^{\mathbb{Z}_{\geq 0}}(j=1, \ldots, 8)$ of poles of $I_{e}(t ; \cdot)$ from the upward sequences $t_{j}^{-1} p^{\mathbb{Z}_{\leq 0}} q^{\mathbb{Z}_{\leq 0}}$ $(j=1, \ldots, 8)$. If the parameters satisfy $\left|t_{j}\right|<1$ this contour can again be taken as the positively oriented unit circle $\mathbb{T}$.

The elliptic hypergeometric function $S_{e}$ extends uniquely to a meromorphic function on $\left\{t \in \mathbb{C}^{8}: \prod t_{j}=p^{2} q^{2}\right\}$. In fact, for a particular value $\tau$ of the parameters for which the integral is not defined, we first deform for $t$ in a small open neighborhood of $\tau$ the contour $\mathcal{C}$ to include those upward poles which collide at $t=\tau$ with downward poles. The resulting expression is an integral which is analytic at an open neighborhood of $\tau$ plus a sum of residues depending meromorphically on the parameters $t$. This expression yields the desired meromorphic extension of $S_{e}(t)$ at $\tau$. For further detailed analysis of meromorphic dependencies of integrals like $S_{e}$, see e.g. [26 and 21].

Since $I_{e}(t ;-z)=I_{e}(-t ; z)$, where $-t=\left(-t_{1}, \ldots,-t_{8}\right)$, we have $S_{e}(t)=S_{e}(-t)$, hence we can and will view $S_{e}$ as a meromorphic function $S_{e}: \mathcal{H}_{p q} \rightarrow \mathbb{C}$. Furthermore, $S_{e}(t)$ is the special case $I I_{\mathrm{BC}}^{1}$ of Rains' [21] multivariate elliptic hypergeometric integrals $I I_{\mathrm{BC}}^{m}$, and it coincides with Spiridonov's $[33, \S 5]$ elliptic analogue $V(\cdot)$ of the Gauss hypergeometric function.

Remark 3.1. Note that $S_{e}(t ; p, q)$ reduces to the elliptic beta integral (3.2) when e.g. $t_{1} t_{6}=p q$. More generally, for e.g. $t_{1} t_{6}=p^{m+1} q^{n+1}\left(m, n \in \mathbb{Z}_{\geq 0}\right)$ it follows from [34, Thm. 11] that $S_{e}(t ; p, q)$ essentially coincides with the two-index elliptic biorthogonal 
rational function $R_{n m}$ of Spiridonov [34, App. A], which is the product of two verywell-poised terminating elliptic hypergeometric ${ }_{12} V_{11}$ series (the second one with the role of the bases $p$ and $q$ reversed).

Next we determine the explicit $W\left(E_{7}\right)$-symmetries of $S_{e}(t)$ in terms of the $W\left(E_{7}\right)$ action on $t \in \mathcal{H}_{p q}$ from Sect. 2. This result was previously obtained by Rains [21] and by Spiridonov [33]. We give here a proof which is similar to Spiridonov's [33, §5] proof.

Theorem 3.2. The elliptic hypergeometric function $S_{e}(t)\left(t \in \mathcal{H}_{p q}\right)$ is invariant under permutations of $\left(t_{1}, \ldots, t_{8}\right)$ and it satisfies

$$
S_{e}(t ; p, q)=S_{e}(w t ; p, q) \prod_{1 \leq j<k \leq 4} \Gamma_{e}\left(t_{j} t_{k} ; p, q\right) \prod_{5 \leq j<k \leq 8} \Gamma_{e}\left(t_{j} t_{k} ; p, q\right)
$$

as meromorphic functions in $t \in \mathcal{H}_{p q}$, where (recall) $w=s_{\beta_{1234}}$.

Proof. The permutation symmetry is trivial. To prove (3.3) we first prove it for parameters $t \in \mathbb{C}^{8}$ satisfying $\prod_{j=1}^{8} t_{j}=p^{2} q^{2}$ and satisfying the additional restraints $\left|t_{j}\right|<1$ $(j=1, \ldots, 8),\left|t_{j}\right|>|p q|^{\frac{1}{3}}(j=5, \ldots, 8)$ and $\left|\prod_{j=5}^{8} t_{j}\right|<|p q|$ (which defines a non-empty open subset of parameters of $\left\{t \in \mathbb{C} \mid \prod_{j=1}^{8} t_{j}=p^{2} q^{2}\right\}$ since $\left.|p|,|q|<1\right)$. For these special values of the parameters we consider the double integral

$$
\int_{\mathbb{T}^{2}} \frac{\prod_{j=1}^{4} \Gamma_{e}\left(t_{j} z^{ \pm 1} ; p, q\right) \Gamma_{e}\left(s x^{ \pm 1} z^{ \pm 1} ; p, q\right) \prod_{j=5}^{8} \Gamma_{e}\left(t_{j} s^{-1} x^{ \pm 1} ; p, q\right)}{\Gamma_{e}\left(z^{ \pm 2}, x^{ \pm 2} ; p, q\right)} \frac{d z}{2 \pi i z} \frac{d x}{2 \pi i x},
$$

where $s$ is chosen to balance both the $z$ as the $x$ integral, so $s^{2} \prod_{j=1}^{4} t_{j}=p q=$ $s^{-2} \prod_{j=5}^{8} t_{j}$. By the additional parameter restraints we have $|s|<1$ and $\left|t_{j} / s\right|<1$ for $j=5, \ldots, 8$, hence the integration contour $\mathbb{T}$ separates the downward pole sequences of the integrand from the upward ones for both integration variables. Using the elliptic beta integral (3.2) to integrate this double integral either first over the variable $z$, or first over the variable $x$, now yields (3.3). Analytic continuation then implies the identity (3.3) as meromorphic functions on $\mathcal{H}_{p q}$.

An interesting equation for $S_{e}(t)$ arises from Theorem 3.2 by considering the action of the longest element $v$ of $W\left(E_{7}\right)$, using its decomposition

$$
v=s_{45} s_{36} s_{48} s_{37} s_{34} s_{12} w_{37} s_{48} w_{35} s_{46} w
$$

as products of permutations and $w$.

Corollary 3.3. We have

$$
S_{e}(t ; p, q)=S_{e}(v t ; p, q) \prod_{1 \leq j<k \leq 8} \Gamma_{e}\left(t_{j} t_{k} ; p, q\right)
$$

as meromorphic functions in $t \in \mathcal{H}_{p q}$.

Remark 3.4. Corollary 3.3 is the special case $n=m=1$ of [21, Thm. 3.1], see also [33, $\S 5$, (iii)] for a proof close to our present derivation. 
3.3. Contiguous relations. For sake of completeness we recall here Spiridonov's [33, §6] derivation of certain contiguous relations cq. difference equations for the elliptic hypergeometric function $S_{e}(t)$ (most notably, Spiridonov's elliptic hypergeometric equation). The starting point is the fundamental theta function identity [5, Exercise 2.16],

$$
\frac{1}{y} \theta\left(u x^{ \pm 1}, y z^{ \pm 1} ; p\right)+\frac{1}{z} \theta\left(u y^{ \pm 1}, z x^{ \pm 1} ; p\right)+\frac{1}{x} \theta\left(u z^{ \pm 1}, x y^{ \pm 1} ; p\right)=0,
$$

which holds for arbitrary $u, x, y, z \in \mathbb{C}^{\times}$. For the $W_{a}\left(E_{7}\right)$-action on $\mathcal{H}_{p q}$ we take in this subsection $\tau_{i j}=\tau_{i j}^{-\log (q)}$, which multiplies $t_{i}$ by $q$ and divides $t_{j}$ by $q$. Note that the $q$-difference operators $\tau_{i j}$ are already well defined on $\left\{t \in \mathbb{C}^{8} \mid \prod_{j=1}^{8} t_{j}=p^{2} q^{2}\right\}$.

Using the difference equation (3.1) of the elliptic gamma function and using (3.6), we have

$$
\frac{\theta\left(q^{-1} t_{8} t_{7}^{ \pm 1} ; p\right)}{\theta\left(t_{6} t_{7}^{ \pm 1} ; p\right)} I_{e}\left(\tau_{68} t ; z\right)+\left(t_{6} \leftrightarrow t_{7}\right)=I_{e}(t ; z)
$$

where $\left(t_{6} \leftrightarrow t_{7}\right)$ means the same term with $t_{6}$ and $t_{7}$ interchanged. For generic $t \in \mathbb{C}^{8}$ with $\prod_{j=1}^{8} t_{j}=p^{2} q^{2}$ we integrate this equality over $z \in \mathcal{C}$, with $\mathcal{C}$ a deformation of $\mathbb{T}$ which separates the upward and downward pole sequences of all three integrands at the same time. We obtain

$$
\frac{\theta\left(q^{-1} t_{8} t_{7}^{ \pm 1} ; p\right)}{\theta\left(t_{6} t_{7}^{ \pm 1} ; p\right)} S_{e}\left(\tau_{68} t\right)+\left(t_{6} \leftrightarrow t_{7}\right)=S_{e}(t)
$$

as meromorphic functions in $t \in \mathcal{H}_{p q}$. This equation is also the $n=1$ instance of [22, Thm. 3.1]. Note that in both terms on the left-hand side the same parameter $t_{8}$ is divided by $q$, while two different parameters $\left(t_{6}\right.$ and $\left.t_{7}\right)$ are multiplied by $q$. We can obtain a different equation (i.e. not obtainable by applying an $S_{8}$ symmetry to (3.7)) by substituting the parameters $v t$ in (3.7), where $v \in W\left(E_{7}\right)$ is the longest Weyl group element, and by using (3.5). The crux is that $\tau_{68} v t=v \tau_{86} t$. We obtain

$$
\frac{\theta\left(t_{7} / q t_{8} ; p\right)}{\theta\left(t_{7} / t_{6} ; p\right)} \prod_{j=1}^{5} \theta\left(t_{j} t_{6} / q ; p\right) S_{e}\left(\tau_{86} t\right)+\left(t_{6} \leftrightarrow t_{7}\right)=\prod_{j=1}^{5} \theta\left(t_{j} t_{8} ; p\right) S_{e}(t)
$$

for $t \in \mathcal{H}_{p q}$. We arrive at Spiridonov's [33, §6] elliptic hypergeometric equation for $S_{e}(t)$.

Theorem 3.5. ([31]) We have

$$
A(t) S_{e}\left(\tau_{87} t ; p, q\right)+\left(t_{7} \leftrightarrow t_{8}\right)=B(t) S_{e}(t ; p, q)
$$

as meromorphic functions in $t \in \mathcal{H}_{p q}$, where $A$ and $B$ are defined by

$$
\begin{aligned}
A(t)= & \frac{1}{t_{8} \theta\left(t_{7} / q t_{8}, t_{8} / t_{7} ; p\right)} \prod_{j=1}^{6} \theta\left(t_{j} t_{7} / q ; p\right), \\
B(t)= & \frac{\theta\left(t_{7} t_{8} / q ; p\right)}{t_{6} \theta\left(t_{7} / q t_{6}, t_{8} / q t_{6} ; p\right)} \prod_{j=1}^{5} \theta\left(t_{j} t_{6} ; p\right)-\frac{\theta\left(t_{6} / t_{8}, t_{6} t_{8} ; p\right)}{t_{6} \theta\left(t_{7} / q t_{6}, t_{7} / q t_{8}, t_{8} / t_{7} ; p\right)} \prod_{j=1}^{5} \theta\left(t_{j} t_{7} / q ; p\right) \\
& -\frac{\theta\left(t_{6} / t_{7}, t_{6} t_{7} ; p\right)}{t_{6} \theta\left(t_{7} / t_{8}, t_{8} / q t_{6}, t_{8} / q t_{7} ; p\right)} \prod_{j=1}^{5} \theta\left(t_{j} t_{8} / q ; p\right) .
\end{aligned}
$$


Remark 3.6. Note that $B$ has an $S_{6}$-symmetry in $\left(t_{1}, t_{2}, \ldots, t_{6}\right)$ even though it is not directly apparent from its explicit representation.

Proof. This follows by taking an appropriate combination of three contiguous relations for $S_{e}(t)$. Specifically, the three contiguous relations are (3.7) and (3.8) with $t_{6}$ and $t_{8}$ interchanged, and (3.7) with $t_{7}$ and $t_{8}$ interchanged.

By combining contiguous relations for $S_{e}(t)$ and exploring the $W\left(E_{7}\right)$-symmetry of $S_{e}(t)$, one can obtain various other contiguous relations involving $S_{e}\left(\tau_{x} t\right), S_{e}\left(\tau_{y} t\right)$, and $S_{e}\left(\tau_{z} t\right)$ for suitable root lattice vectors $x, y, z \in L$. A detailed analysis of such procedures is undertaken for three term transformation formulas on the trigonometric setting by Lievens and Van der Jeugt [15] (see also Sect. 5).

Remark 3.7. Interchanging the role of the bases $p$ and $q$ and using the symmetry of $S_{e}(t ; p, q)$ in $p$ and $q$, we obtain contiguous relations for $S_{e}(t ; p, q)$ with respect to multiplicative $p$-shifts in the parameters.

\section{Hyperbolic Hypergeometric Integrals}

4.1. The hyperbolic gamma function. We fix throughout this section $\omega_{1}, \omega_{2} \in \mathbb{C}$ satisfying $\Re\left(\omega_{1}\right), \Re\left(\omega_{2}\right)>0$, and we write

$$
\omega=\frac{\omega_{1}+\omega_{2}}{2} .
$$

Ruijsenaars' [24] hyperbolic gamma function is defined by

$$
G\left(z ; \omega_{1}, \omega_{2}\right)=\exp \left(i \int_{0}^{\infty}\left(\frac{\sin (2 z t)}{2 \sinh \left(\omega_{1} t\right) \sinh \left(\omega_{2} t\right)}-\frac{z}{\omega_{1} \omega_{2} t}\right) \frac{d t}{t}\right)
$$

for $z \in \mathbb{C}$ satisfying $|\Im(z)|<\Re(\omega)$. There exists a unique meromorphic extension of $G\left(\omega_{1}, \omega_{2} ; z\right)$ to $z \in \mathbb{C}$ satisfying

$$
\begin{aligned}
G\left(z ; \omega_{1}, \omega_{2}\right) & =G\left(z ; \omega_{2}, \omega_{1}\right), \\
G\left(z ; \omega_{1}, \omega_{2}\right) & =G\left(-z ; \omega_{1}, \omega_{2}\right)^{-1}, \\
G\left(z+i \omega_{1} ; \omega_{1}, \omega_{2} ;\right) & =-2 i s\left((z+i \omega) / \omega_{2}\right) G\left(z ; \omega_{1}, \omega_{2}\right),
\end{aligned}
$$

where we use the shorthand notation $s(z)=\sinh (\pi z)$. The second equation here is called the reflection equation. In this section we will omit the $\omega_{1}, \omega_{2}$ dependence of $G$ if no confusion is possible, and we formulate all results only with respect to $i \omega_{1}$-shifts. We use similar notations for products of hyperbolic gamma functions as for $q$-shifted factorials and elliptic gamma functions, e.g.

$$
G\left(z_{1}, \ldots, z_{n} ; \omega_{1}, \omega_{2}\right)=\prod_{j=1}^{n} G\left(z_{j} ; \omega_{1}, \omega_{2}\right)
$$
$\Gamma_{e}$,

The hyperbolic gamma function $G$ is a degeneration of the elliptic gamma function

$$
\begin{aligned}
& \lim _{r \searrow 0} \Gamma_{e}\left(\exp (2 \pi i r z) ; \exp \left(-2 \pi \omega_{1} r\right), \exp \left(-2 \pi \omega_{2} r\right)\right) \exp \left(\frac{\pi(z-i \omega)}{6 i r \omega_{1} \omega_{2}}\right) \\
& \quad=G\left(z-i \omega ; \omega_{1}, \omega_{2}\right)
\end{aligned}
$$

for $\omega_{1}, \omega_{2}>0$, see [24, Prop. III.12]. 
In this section we call a sequence of points a downward (respectively upward) sequence of points if it is of the form $a+i \mathbb{Z}_{\leq 0} \omega_{1}+i \mathbb{Z}_{\leq 0} \omega_{2}$ (respectively $a+i \mathbb{Z}_{\geq 0} \omega_{1}+$ $\left.i \mathbb{Z}_{>0} \omega_{2}\right)$ for some $a \in \mathbb{C}$. Recall from [24] that the hyperbolic gamma function $G\left(\omega_{1}, \omega_{2} ; z\right)$, viewed as a meromorphic function in $z \in \mathbb{C}$, has poles at the downward sequence $-i \omega+i \mathbb{Z}_{\leq 0} \omega_{1}+i \mathbb{Z}_{\leq 0} \omega_{2}$ of points and has zeros at the upward sequence $i \omega+i \mathbb{Z}_{\geq 0} \omega_{1}+i \mathbb{Z}_{\geq 0} \omega_{2}$ of points. The pole of $G\left(z ; \omega_{1}, \omega_{2}\right)$ at $z=-i \omega$ is simple and

$$
\underset{z=-i \omega}{\operatorname{Res}}\left(G\left(z ; \omega_{1}, \omega_{2}\right)\right)=\frac{i}{2 \pi} \sqrt{\omega_{1} \omega_{2}} .
$$

All contours in this section will be chosen as deformations of the real line $\mathbb{R}$ separating the upward pole sequences of the integrand from the downward ones.

We will also need to know the asymptotic behavior of $G(z)$ as $\Re(z) \rightarrow \pm \infty$ (uniformly for $\Im(z)$ in compacta of $\mathbb{R})$. For our purposes it is sufficient to know that for any $a, b \in \mathbb{C}$ we have

$$
\lim _{\Re(z) \rightarrow \infty} \frac{G\left(z-a ; \omega_{1}, \omega_{2}\right)}{G\left(z-b ; \omega_{1}, \omega_{2}\right)} \exp \left(\frac{\pi i z}{\omega_{1} \omega_{2}}(b-a)\right)=\exp \left(\frac{\pi i}{2 \omega_{1} \omega_{2}}\left(b^{2}-a^{2}\right)\right),
$$

where the corresponding $o(\Re(z))$-tail as $\Re(z) \rightarrow \infty$ can be estimated uniformly for $\Im(z)$ in compacta of $\mathbb{R}$, and that for periods satisfying $\omega_{1} \omega_{2} \in \mathbb{R}_{>0}$,

$$
\left|G\left(u+x ; \omega_{1}, \omega_{2}\right)\right| \leq M \exp \left(\pi \Im\left(\frac{u}{\omega_{1} \omega_{2}}\right)|x|\right), \quad \forall x \in \mathbb{R}
$$

for some constant $M>0$, provided that the line $u+\mathbb{R}$ does not hit a pole of $G$. See [26, App. A] for details and for more precise asymptotic estimates.

4.2. Symmetries of the hyperbolic hypergeometric function. The univariate hyperbolic beta integral $[37,(1.10)]$ is

$$
\int_{\mathcal{C}} \frac{G\left(i \omega \pm 2 z ; \omega_{1}, \omega_{2}\right)}{\prod_{j=1}^{6} G\left(u_{j} \pm z ; \omega_{1}, \omega_{2}\right)} d z=2 \sqrt{\omega_{1} \omega_{2}} \prod_{1 \leq j<k \leq 6} G\left(i \omega-u_{j}-u_{k} ; \omega_{1}, \omega_{2}\right)
$$

for generic $u_{1}, \ldots, u_{6} \in \mathbb{C}$ satisfying the additive balancing condition $\sum_{j=1}^{6} u_{j}=4 i \omega$. Note that this integral converges since the asymptotic behaviour of the integrand at $z= \pm \infty$ is $\mathcal{O}\left(\exp \left(-4 \pi|z| \omega / \omega_{1} \omega_{2}\right)\right)$ in view of the reflection equation (4.1), the limit (4.4) and the fact that $\Re\left(\frac{\omega}{\omega_{1} \omega_{2}}\right)>0$ due to the imposed conditions $\Re\left(\omega_{j}\right)>0$ on the periods $\omega_{j}(j=1,2)$.

We define now the integrand of the hyperbolic hypergeometric function $I_{h}(u ; z)=$ $I_{h}\left(u ; z ; \omega_{1}, \omega_{2}\right)$ as

$$
I_{h}\left(u ; z ; \omega_{1}, \omega_{2}\right)=\frac{G\left(i \omega \pm 2 z ; \omega_{1}, \omega_{2}\right)}{\prod_{j=1}^{8} G\left(u_{j} \pm z ; \omega_{1}, \omega_{2}\right)}
$$

for parameters $u \in \mathbb{C}^{8}$. The hyperbolic hypergeometric function $S_{h}(u)=S_{h}\left(u ; \omega_{1}, \omega_{2}\right)$ is defined by

$$
S_{h}\left(u ; \omega_{1}, \omega_{2}\right)=\int_{\mathcal{C}} I_{h}\left(u ; z ; \omega_{1}, \omega_{2}\right) d z
$$


for generic parameters $u \in \mathcal{G}_{2 i \omega}$ (see Sect. 2 for the definition of $\mathcal{G}_{2 i \omega}$ ). The asymptotic behaviour of $I_{h}(u ; z)$ at $z= \pm \infty$ is again $\mathcal{O}\left(\exp \left(-4 \pi|z| \omega / \omega_{1} \omega_{2}\right)\right)$, so the integral absolutely converges. It follows from (4.3) and the analytic difference equations for the hyperbolic gamma function that $S_{h}(u)$ has a unique meromorphic extension to $u \in \mathcal{G}_{2 i \omega}$, cf. the analysis for the elliptic hypergeometric function $S_{e}(t)$. We thus can and will view $S_{h}(u)$ as a meromorphic function in $u \in \mathcal{G}_{2 i \omega}$. Note furthermore that the real line can be chosen as an integration contour in the definition of $S_{h}(u)$ if $u \in \mathcal{G}_{2 i \omega}$ satisfies $\Im\left(u_{j}-i \omega\right)<0$ for all $j$. The hyperbolic hypergeometric function $S_{h}(u)\left(u \in \mathcal{G}_{2 i \omega}\right)$ coincides with Spiridonov's [33, §5] hyperbolic analogue $s(\cdot)$ of the Gauss hypergeometric function.

Using (4.2) and the reflection equation of $G$, we can obtain the hyperbolic hypergeometric function $S_{h}\left(v u ; \omega_{1}, \omega_{2}\right)=S_{h}\left(i \omega-u_{1}, \ldots, i \omega-u_{8} ; \omega_{1}, \omega_{2}\right)\left(u \in \mathcal{G}_{2 i \omega}\right)$ formally as the degeneration $r \downarrow 0$ of the elliptic hypergeometric function $S_{e}(t ; p, q)$ with $p=\exp \left(-2 \pi \omega_{1} r\right), q=\exp \left(-2 \pi \omega_{2} r\right)$ and $t=\psi_{2 i \omega}(2 \pi i r u) \in \mathcal{H}_{\exp (-4 \pi r \omega)}=\mathcal{H}_{p q}$. This degeneration, which turns out to preserve the $W\left(E_{7}\right)$-symmetry (see below), can be proven rigorously, see [23]. This entails in particular a derivation of the hyperbolic beta integral (4.6) as a rigorous degeneration of the elliptic beta integral (3.2) (see [37, $\S 5.4]$ for the formal analysis).

Next we give the explicit $W\left(E_{7}\right)$ symmetries of $S_{h}(u)$ in terms of the $W\left(E_{7}\right)$ action on $u \in \mathcal{G}_{2 i \omega}$ from Sect. 2 .

Theorem 4.1. The hyperbolic hypergeometric function $S_{h}(u)\left(u \in \mathcal{G}_{2 i \omega}\right)$ is invariant under permutations of $\left(u_{1}, \ldots, u_{8}\right)$ and it satisfies

$$
\begin{aligned}
S_{h}\left(u ; \omega_{1}, \omega_{2}\right)= & S_{h}\left(w u ; \omega_{1}, \omega_{2}\right) \prod_{1 \leq j<k \leq 4} G\left(i \omega-u_{j}-u_{k} ; \omega_{1}, \omega_{2}\right) \\
& \times \prod_{5 \leq j<k \leq 8} G\left(i \omega-u_{j}-u_{k} ; \omega_{1}, \omega_{2}\right)
\end{aligned}
$$

as meromorphic functions in $u \in \mathcal{G}_{2 i \omega}$.

Proof. The proof is analogous to the proof in the elliptic case (Theorem 3.2). For the $w$-symmetry we consider for suitable $u \in \mathcal{G}_{2 i \omega}$ the double integral

$$
\int_{\mathbb{R}^{2}} \frac{G(i \omega \pm 2 z, i \omega \pm 2 x)}{\prod_{j=1}^{4} G\left(u_{j} \pm z\right) G(i \omega+s \pm x \pm z) \prod_{k=5}^{8} G\left(u_{k}-s \pm x\right)} d z d x
$$

with $s=i \omega-\frac{1}{2}\left(u_{1}+u_{2}+u_{3}+u_{4}\right)=-i \omega+\frac{1}{2}\left(u_{5}+u_{6}+u_{7}+u_{8}\right)$. We impose the conditions $\Im(s)<0$ and

$$
\mathfrak{\Im}\left(u_{j}-i \omega\right)<0 \quad(j=1, \ldots, 4), \quad \Im\left(u_{k}-i \omega\right)<\Im(s) \quad(k=5, \ldots, 8)
$$

on $u \in \mathcal{G}_{2 i \omega}$ to ensure that the upward and downward pole sequences of the integrand of the double integral are separated by $\mathbb{R}$. Next we show that the parameter restraints

$$
-\mathfrak{R}\left(\frac{\omega}{\omega_{1} \omega_{2}}\right)<\Im\left(\frac{s}{\omega_{1} \omega_{2}}\right)<0
$$

on $u \in \mathcal{G}_{2 i \omega}$ suffice to ensure absolute convergence of the double integral. Using the reflection equation and asymptotics (4.5) of $G$ we obtain the estimate

$$
\frac{1}{|G(i \omega+s \pm x \pm z)|} \leq M \exp \left(-2 \pi \Im\left(\frac{s+i \omega}{\omega_{1} \omega_{2}}\right)(|z+x|+|z-x|)\right), \quad \forall(x, z) \in \mathbb{R}^{2}
$$


for some constant $M>0$. It follows that the factor $G(i \omega+s \pm x \pm z)^{-1}$ of the integrand is absolutely and uniformly bounded if $\Im\left((i \omega+s) / \omega_{1} \omega_{2}\right) \geq 0$, i.e. if $\Im\left(s / \omega_{1} \omega_{2}\right) \geq$ $-\Re\left(\omega / \omega_{1} \omega_{2}\right)$. The asymptotic behaviour of the remaining factors of the integrand (which breaks up in factors only depending on $x$ or on $z$ ) can easily be determined by (4.5), leading finally to the parameter restraints (4.8) for the absolute convergence of the double integral.

It is easy to verify that the parameter subset of $\mathcal{G}_{2 i \omega}$ defined by the additional restraints $\Im(s)<0,(4.7)$ and (4.8) is non-empty (by e.g. constructing parameters $u \in \mathcal{G}_{2 i \omega}$ with small associated balancing parameter $s$ ). Using Fubini's Theorem and the hyperbolic beta integral (4.6), we now reduce the double integral to a single integral by either evaluating the integral over $x$, or by evaluating the integral over $z$. Using furthermore that

$$
w u=\left(u_{1}+s, u_{2}+s, u_{3}+s, u_{4}+s, u_{5}-s, u_{6}-s, u_{7}-s, u_{8}-s\right)
$$

for $u \in \mathcal{G}_{2 i \omega}$, it follows that the resulting identity is the desired $w$-symmetry of $S_{h}$ for the restricted parameter domain. Analytic continuation now completes the proof.

The symmetry of $S_{h}(u)\left(u \in \mathcal{G}_{2 i \omega}\right)$ with respect to the action of the longest Weyl group element $v \in W\left(E_{7}\right)$ is as follows.

Corollary 4.2. The hyperbolic hypergeometric function $S_{h}$ satisfies

$$
S_{h}\left(u ; \omega_{1}, \omega_{2}\right)=S_{h}\left(v u ; \omega_{1}, \omega_{2}\right) \prod_{1 \leq j<k \leq 8} G\left(i \omega-u_{j}-u_{k} ; \omega_{1}, \omega_{2}\right)
$$

as meromorphic functions in $u \in \mathcal{G}_{2 i \omega}$.

Proof. This follows from Theorem 4.1 and (3.4).

4.3. Contiguous relations. Contiguous relations for the hyperbolic hypergeometric function $S_{h}$ can be derived in nearly exactly the same manner as we did for the elliptic hypergeometric function $S_{e}$ (see Sect. 3.3 and $[33, \S 6]$ ). We therefore only indicate the main steps. Using the $p=0$ case of (3.6) we have

$$
s(x \pm v) s(y \pm z)+s(x \pm y) s(z \pm v)+s(x \pm z) s(v \pm y)=0,
$$

where $s(x \pm v)=s(x+v) s(x-v)$. In this subsection we write $\tau_{j k}=\tau_{j k}^{i \omega_{1}}(1 \leq j \neq$ $k \leq 8)$, which acts on $u \in \mathcal{G}_{2 i \omega}$ by subtracting $i \omega_{1}$ from $u_{j}$ and adding $i \omega_{1}$ to $u_{k}$. We now obtain in analogy to the elliptic case the difference equation

$$
\frac{s\left(\left(u_{8}+i \omega \pm\left(u_{7}-i \omega\right)\right) / \omega_{2}\right)}{s\left(\left(u_{6}-i \omega \pm\left(u_{7}-i \omega\right)\right) / \omega_{2}\right)} S_{h}\left(\tau_{68} u\right)+\left(u_{6} \leftrightarrow u_{7}\right)=S_{h}(u)
$$

as meromorphic functions in $u \in \mathcal{G}_{2 i \omega}$. Using (4.9) we subsequently obtain

$$
\begin{aligned}
& \frac{s\left(\left(u_{7}-u_{8}+2 i \omega\right) / \omega_{2}\right)}{s\left(\left(u_{7}-u_{6}\right) / \omega_{2}\right)} \prod_{j=1}^{5} s\left(\left(u_{j}+u_{6}\right) / \omega_{2}\right) S_{h}\left(\tau_{86} u\right)+\left(u_{6} \leftrightarrow u_{7}\right) \\
& \quad=\prod_{j=1}^{5} s\left(\left(u_{j}+u_{8}-2 i \omega\right) / \omega_{2}\right) S_{h}(u)
\end{aligned}
$$


as meromorphic functions in $u \in \mathcal{G}_{2 i \omega}$. Combining these contiguous relations and simplifying we obtain

$$
A(u) S_{h}\left(\tau_{87} u\right)-\left(u_{7} \leftrightarrow u_{8}\right)=B(u) S_{h}(u), \quad u \in \mathcal{G}_{2 i \omega},
$$

where

$$
\begin{aligned}
A(u)= & s\left(\left(2 i \omega-u_{7}+u_{8}\right) / \omega_{2}\right) \prod_{j=1}^{6} s\left(\left(u_{j}+u_{7}\right) / \omega_{2}\right), \\
B(u)= & \frac{s\left(\left(u_{8} \pm u_{7}\right) / \omega_{2}\right) s\left(\left(2 i \omega+u_{8}-u_{7}\right) / \omega_{2}\right) s\left(\left(2 i \omega-u_{8}+u_{7}\right) / \omega_{2}\right)}{s\left(\left(2 i \omega+u_{8}-u_{6}\right) / \omega_{2}\right) s\left(\left(2 i \omega+u_{7}-u_{6}\right) / \omega_{2}\right)} \\
& \times \prod_{j=1}^{5} s\left(\left(-2 i \omega+u_{j}+u_{6}\right) / \omega_{2}\right) \\
& -\frac{s\left(\left(2 i \omega-u_{8}+u_{7}\right) / \omega_{2}\right) s\left(\left(u_{7}-u_{6}\right) / \omega_{2}\right) s\left(\left(-2 i \omega+u_{6}+u_{7}\right) / \omega_{2}\right)}{s\left(\left(2 i \omega+u_{8}-u_{6}\right) / \omega_{2}\right)} \\
& \times \prod_{j=1}^{5} s\left(\left(u_{j}+u_{8}\right) / \omega_{2}\right) \\
& +\frac{s\left(\left(2 i \omega+u_{8}-u_{7}\right) / \omega_{2}\right) s\left(\left(u_{8}-u_{6}\right) / \omega_{2}\right) s\left(\left(-2 i \omega+u_{6}+u_{8}\right) / \omega_{2}\right)}{s\left(\left(2 i \omega+u_{7}-u_{6}\right) / \omega_{2}\right)} \\
& \times \prod_{j=1}^{5} s\left(\left(u_{j}+u_{7}\right) / \omega_{2}\right) .
\end{aligned}
$$

This leads to the following theorem.

Theorem 4.3. We have

$$
A(u)\left(S_{h}\left(\tau_{87} u\right)-S_{h}(u)\right)-\left(u_{7} \leftrightarrow u_{8}\right)=B_{2}(u) S_{h}(u)
$$

as meromorphic functions in $u \in \mathcal{G}_{2 i \omega}$, where $A(u)$ is as above and with $B_{2}(u)$ defined by

$$
\begin{aligned}
B_{2}(u)= & \frac{s\left(\left(u_{7} \pm u_{8}\right) / \omega_{2}\right) s\left(\left(u_{7}-u_{8} \pm 2 i \omega\right) / \omega_{2}\right)}{4} \\
& \times\left(\sum_{j=7}^{8} s\left(2\left(i \omega+u_{j}\right) / \omega_{2}\right)-\sum_{j=1}^{6} s\left(2\left(i \omega-u_{j}\right) / \omega_{2}\right)\right) .
\end{aligned}
$$

Proof. It follows from (4.10) that (4.11) holds with $B_{2}(u)=B(u)-A(u)-A\left(s_{78} u\right)$. The alternative expression (4.12) for $B_{2}$ was obtained by Mathematica. Observe though that part of the zero locus of $B_{2}(u)\left(u \in \mathcal{G}_{2 i \omega}\right)$ can be predicted in advance. Indeed, the left-hand side of (4.11) vanishes if $u_{7}=u_{8}$ (both terms then cancel each other), and it vanishes if $u_{7}=u_{8} \pm i \omega$ (one term vanishes due to an $s$-factor, while the other term vanishes since either $S_{h}\left(\tau_{87} u\right)=S_{h}(u)$ or $\left.S_{h}\left(\tau_{78} u\right)=S_{h}(u)\right)$. The zero of $B_{2}(u)$ at $u_{7}=-u_{8}$ can be predicted from the fact that all hyperbolic hypergeometric functions $S_{h}$ in (4.11) can be evaluated for $u_{7}=-u_{8}$ using the hyperbolic beta integral (4.6). 
4.4. The degeneration to the hyperbolic Barnes integral. In this subsection we degenerate the hyperbolic hypergeometric function $S_{h}(u)\left(u \in \mathcal{G}_{2 i \omega}\right)$ along the highest root $\beta_{1278}$ of $R\left(E_{7}\right)$ with respect to the basis $\Delta_{2}$ of $R\left(E_{7}\right)$ (see (2.4) for the associated Dynkin diagram). The resulting degenerate integral $B_{h}(u)$ thus inherits symmetries with respect to the standard maximal parabolic subgroup

$$
W_{2}\left(D_{6}\right):=W\left(E_{7}\right)_{\beta_{1278}} \subset W\left(E_{7}\right),
$$

which is isomorphic to the Weyl group of type $D_{6}$ and is generated by the simple reflections $s_{\alpha}\left(\alpha \in \Delta_{2} \backslash\left\{\alpha_{23}^{-}\right\}\right)$. The corresponding Dynkin diagram is

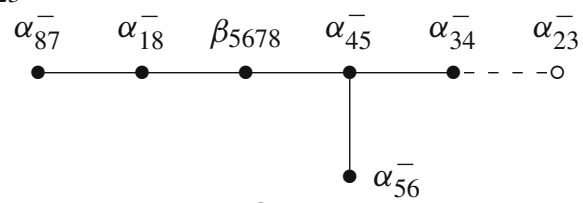

Concretely, for generic parameters $u \in \mathcal{G}_{2 i \omega}$ we define $B_{h}(u)=B_{h}\left(u ; \omega_{1}, \omega_{2}\right)$ by

$$
B_{h}\left(u ; \omega_{1}, \omega_{2}\right)=2 \int_{\mathcal{C}} \frac{\prod_{j=3}^{6} G\left(z-u_{j} ; \omega_{1}, \omega_{2}\right)}{\prod_{j=1,2,7,8} G\left(z+u_{j} ; \omega_{1}, \omega_{2}\right)} d z .
$$

This integral converges absolutely since the asymptotic behaviour of the integrand at $z= \pm \infty$ is $\exp \left(-4 \pi \omega|z| / \omega_{1} \omega_{2}\right)$. We may take the real line as integration contour if $u \in \mathcal{G}_{2 i \omega}$ satisfies $\Im\left(u_{j}-i \omega\right)<0$ for all $j$. Observe that the integral $B_{h}(u)$ has a unique meromorphic extension to $u \in \mathcal{G}_{2 i \omega}$. We call $B_{h}(u)$ the hyperbolic Barnes integral since it is essentially Ruijsenaars' [26] hyperbolic generalization of the Barnes integral representation of the Gauss hypergeometric function, see Subsect. 4.6.

Remark 4.4. The parameter space of the hyperbolic Barnes integral $B_{h}$ is in fact the quotient space $\mathcal{G}_{2 i \omega} / \mathbb{C} \beta_{1278}$. Indeed, for $\xi \in \mathbb{C}$ we have

$$
B_{h}\left(u+\xi \beta_{1278}\right)=B_{h}(u)
$$

as meromorphic functions in $u \in \mathcal{G}_{2 i \omega}$, which follows by an easy application of (4.4) and Cauchy's Theorem.

Proposition 4.5. For $u \in \mathcal{G}_{2 i \omega}$ satisfying $\Im\left(u_{j}-i \omega\right)<0(j=1, \ldots, 8)$ we have

$$
\lim _{r \rightarrow \infty} S_{h}\left(u-r \beta_{1278}\right) \exp \left(\frac{2 \pi r \omega}{\omega_{1} \omega_{2}}\right) \exp \left(\frac{\pi i}{2 \omega_{1} \omega_{2}}\left(\sum_{j=1,2,7,8} u_{j}^{2}-\sum_{j=3}^{6} u_{j}^{2}\right)\right)=B_{h}(u) .
$$

Proof. The conditions on the parameters $u \in \mathcal{G}_{2 i \omega}$ allow us to choose the real line as an integration contour in the integral expression of $S_{h}\left(u-r \beta_{1278}\right)(r \in \mathbb{R})$ as well as in the integral expression of $B_{h}(u)$. Using that the integrand $I_{h}(u ; z)$ of $S_{h}(u)$ is even in $x$, using the reflection equation for the hyperbolic gamma function, and by a change of integration variable, we have

$$
\begin{aligned}
S_{h}\left(u-r \beta_{1278}\right) e^{\frac{2 \pi r \omega}{\omega_{1} \omega_{2}}} & =e^{\frac{2 \pi r \omega}{\omega_{1} \omega_{2}}} \int_{-\infty}^{\infty} \frac{G(i \omega \pm 2 z)}{\prod_{j=1,2,7,8} G\left(u_{j}-\frac{r}{2} \pm z\right) \prod_{j=3}^{6} G\left(u_{j}+\frac{r}{2} \pm z\right)} d z \\
& =2 e^{\frac{2 \pi r \omega}{\omega_{1} \omega_{2}}} \int_{0}^{\infty} \frac{G(i \omega \pm 2 z)}{\prod_{j=1,2,7,8} G\left(u_{j}-\frac{r}{2} \pm z\right) \prod_{j=3}^{6} G\left(u_{j}+\frac{r}{2} \pm z\right)} d z \\
& =2 \int_{-\frac{r}{2}}^{\infty} k_{1}(2 z+r) k_{2}(z+r) L(z) d z,
\end{aligned}
$$


where

$$
\begin{aligned}
L(z) & =\frac{\prod_{j=3}^{6} G\left(z-u_{j}\right)}{\prod_{j=1,2,7,8} G\left(z+u_{j}\right)}, \\
k_{1}(z) & =\frac{G(z+i \omega)}{G(z-i \omega)} e^{-\frac{2 \pi \omega z}{\omega_{1} \omega_{2}}}=\left(1-e^{-2 \pi z / \omega_{1}}\right)\left(1-e^{-2 \pi z / \omega_{2}}\right), \\
k_{2}(z) & =\frac{\prod_{j=1,2,7,8} G\left(z-u_{j}\right)}{\prod_{j=3}^{6} G\left(z+u_{j}\right)} e^{\frac{4 \pi \omega z}{\omega_{1} \omega_{2}}} .
\end{aligned}
$$

Here the second expression of $k_{1}$ follows from the analytic difference equations satisfied by $G$. The pointwise limits of $k_{1}$ and $k_{2}$ are

$$
\lim _{z \rightarrow \infty} k_{1}(z)=1 \quad \lim _{z \rightarrow \infty} k_{2}(z)=e^{\frac{\pi i}{2 \omega_{1} \omega_{2}}\left(\sum_{j=3}^{6} u_{j}^{2}-\sum_{j=1,2,7,8} u_{j}^{2}\right)} .
$$

Moreover, observe that $k_{1}(z)$ is uniformly bounded for $z \in \mathbb{R}_{\geq 0}$ by 4 , and that $k_{2}(z)$, being a continuous function on $\mathbb{R}_{\geq 0}$ with finite limit at infinity, is also uniformly bounded for $z \in \mathbb{R}_{\geq 0}$.

Denote by $\chi_{(-r / 2, \infty)}(z)$ the indicator function of the interval $(-r / 2, \infty)$. By Lebesgue's theorem of dominated convergence we now conclude that

$$
\begin{aligned}
& \lim _{r \rightarrow \infty} S_{h}\left(u-r \beta_{1278}\right) e^{\frac{2 \pi r \omega}{\omega_{1} \omega_{2}}}=2 \lim _{r \rightarrow \infty} \int_{-\frac{r}{2}}^{\infty} k_{1}(2 z+r) k_{2}(z+r) L(z) d z \\
& =2 \int_{-\infty}^{\infty} \lim _{r \rightarrow \infty} \chi_{(-r / 2, \infty)}(z) k_{1}(2 z+s) k_{2}(z+s) L(z) d z \\
& =2 e^{\frac{\pi i}{2 \omega_{1} \omega_{2}}}\left(\sum_{j=3}^{6} u_{j}^{2}-\sum_{j=1,2,7,8} u_{j}^{2}\right) \int_{-\infty}^{\infty} L(z) d z \\
& =e^{\frac{\pi i}{2 \omega_{1} \omega_{2}}\left(\sum_{j=3}^{6} u_{j}^{2}-\sum_{j=1,2,7,8} u_{j}^{2}\right)} B_{h}(u),
\end{aligned}
$$

as desired.

In the following corollary we use Proposition 4.5 to degenerate the hyperbolic beta integral (4.6). The resulting integral evaluation formula is an hyperbolic analogue of the nonterminating Saalschütz formula [5, (2.10.12)], see Subsect. 5.4.

Corollary 4.6. For generic $u \in \mathbb{C}^{6}$ satisfying $\sum_{j=1}^{6} u_{j}=4 i \omega$ we have

$$
\int_{\mathcal{C}} \frac{G\left(z-u_{4}, z-u_{5}, z-u_{6}\right)}{G\left(z+u_{1}, z+u_{2}, z+u_{3}\right)} d z=\sqrt{\omega_{1} \omega_{2}} \prod_{j=1}^{3} \prod_{k=4}^{6} G\left(i \omega-u_{j}-u_{k}\right)
$$

Proof. Substitute the parameters $u^{\prime}=\left(u_{1}, u_{2}, u_{4}, u_{5}, u_{6}, 0, u_{3}, 0\right)$ in Proposition 4.5 with $u_{j} \in \mathbb{C}$ satisfying $\Im\left(u_{j}-i \omega\right)<0$ and $\sum_{j=1}^{6} u_{j}=4 i \omega$. Then $B_{h}\left(u^{\prime}\right)$ is the lefthand side of (4.13), multiplied by 2. On the other hand, by Proposition 4.5 and (4.6) we have 


$$
\begin{aligned}
& B_{h}\left(u^{\prime}\right)=\lim _{r \rightarrow \infty} S_{h}\left(u^{\prime}-r \beta_{1278}\right) \exp \left(\frac{2 \pi r \omega}{\omega_{1} \omega_{2}}+\frac{\pi i}{2 \omega_{1} \omega_{2}}\left(\sum_{j=1}^{3} u_{j}^{2}-\sum_{j=4}^{6} u_{j}^{2}\right)\right) \\
& =2 \sqrt{\omega_{1} \omega_{2}} \prod_{j=1}^{3} \prod_{k=4}^{6} G\left(i \omega-u_{j}-u_{k}\right) \\
& \quad \times \lim _{r \rightarrow \infty} \frac{\prod_{1 \leq j<k \leq 3} G\left(i \omega-u_{j}-u_{k}+r\right)}{\prod_{4 \leq j<k \leq 6} G\left(u_{j}+u_{k}-i \omega+r\right)} \exp \left(\frac{2 \pi r \omega}{\omega_{1} \omega_{2}}+\frac{\pi i}{2 \omega_{1} \omega_{2}}\left(\sum_{j=1}^{3} u_{j}^{2}-\sum_{j=4}^{6} u_{j}^{2}\right)\right) \\
& =2 \sqrt{\omega_{1} \omega_{2}} \prod_{j=1}^{3} \prod_{k=4}^{6} G\left(i \omega-u_{j}-u_{k}\right),
\end{aligned}
$$

where the last equality follows from a straightforward but tedious computation using (4.4). The result for arbitrary generic parameters $u \in \mathbb{C}^{6}$ satisfying $\sum_{j=1}^{6} u_{j}=4 i \omega$ now follows by analytic continuation.

Next we determine the explicit $W_{2}\left(D_{6}\right)$-symmetries of $B_{h}(u)$.

Proposition 4.7. The hyperbolic Barnes integral $B_{h}(u)\left(u \in \mathcal{G}_{2 i \omega}\right)$ is invariant under permutations of $\left(u_{1}, u_{2}, u_{7}, u_{8}\right)$ and of $\left(u_{3}, u_{4}, u_{5}, u_{6}\right)$ and it satisfies

$$
B_{h}(u)=B_{h}(w u) \prod_{j=1,2} \prod_{k=3,4} G\left(i \omega-u_{j}-u_{k}\right) \prod_{j=5,6} \prod_{k=7,8} G\left(i \omega-u_{j}-u_{k}\right)
$$

as meromorphic functions in $u \in \mathcal{G}_{2 i \omega}$.

Proof. The permutation symmetry is trivial. The symmetry (4.14) can be proven by degenerating the corresponding symmetry of $S_{h}$, see Theorem 4.1. We prove here the $w$-symmetry by considering the double integral

$$
\int_{\mathbb{R}^{2}} \frac{G\left(z-u_{3}, z-u_{4}, x-u_{5}+s, x-u_{6}+s, z-x-i \omega-s\right)}{G\left(z+u_{1}, z+u_{2}, x+u_{7}-s, x+u_{8}-s, z-x+i \omega+s\right)} d z d x
$$

with $s=i \omega-\frac{1}{2}\left(u_{1}+u_{2}+u_{3}+u_{4}\right)=-i \omega+\frac{1}{2}\left(u_{5}+u_{6}+u_{7}+u_{8}\right)$, where we impose on $u \in \mathcal{G}_{2 i \omega}$ the additional conditions

$$
-\Re\left(\frac{\omega}{\omega_{1} \omega_{2}}\right)<\Im\left(\frac{s}{\omega_{1} \omega_{2}}\right)<\Re\left(\frac{\omega}{\omega_{1} \omega_{2}}\right)
$$

to ensure the absolute convergence of the double integral (this condition is milder than the corresponding condition (4.8) for $S_{h}$ due to the missing factors $G(i \omega \pm 2 z, i \omega \pm 2 x)$ in the numerator of the integrand), and the conditions (4.7) to ensure that the upward and downward pole sequences are separated by $\mathbb{R}$. Using Fubini's Theorem and the hyperbolic Saalschütz summation (4.13), similarly as in the proof of Theorem 4.1, yields (4.14). 
4.5. The degeneration to the hyperbolic Euler integral. In this subsection we degenerate the hyperbolic hypergeometric function $S_{h}(u)\left(u \in \mathcal{G}_{2 i \omega}\right)$ along the highest root $\alpha_{78}^{-}$of $R\left(E_{7}\right)$ with respect to the basis $\Delta_{1}$ of $R\left(E_{7}\right)$ (see (2.3) for the associated Dynkin diagram). The resulting degenerate integral $E_{h}(u)$ thus inherits symmetries with respect to the standard maximal parabolic subgroup

$$
W_{1}\left(D_{6}\right):=W\left(E_{7}\right)_{\alpha_{78}^{-}} \subset W\left(E_{7}\right),
$$

which is isomorphic to the Weyl group of type $D_{6}$ and is generated by the simple reflections $s_{\alpha}\left(\alpha \in \Delta_{1} \backslash\left\{\alpha_{76}^{-}\right\}\right)$. The corresponding Dynkin diagram is

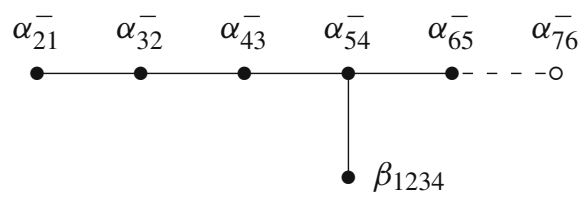

By the conditions $\mathfrak{R}\left(\omega_{j}\right)>0$ on the periods $\omega_{j}(j=1,2)$ we have that $\mathfrak{R}\left(\frac{\omega}{\omega_{1} \omega_{2}}\right)>0$. For generic parameters $u=\left(u_{1}, \ldots, u_{6}\right) \in \mathbb{C}^{6}$ satisfying

$$
\Im\left(\frac{1}{\omega_{1} \omega_{2}} \sum_{j=1}^{6} u_{j}\right)>\Re\left(\frac{2 \omega}{\omega_{1} \omega_{2}}\right)
$$

we now define $E_{h}(u)=E_{h}\left(u ; \omega_{1}, \omega_{2}\right)$ by

$$
E_{h}\left(u ; \omega_{1}, \omega_{2}\right)=\int_{\mathcal{C}} \frac{G\left(i \omega \pm 2 z ; \omega_{1}, \omega_{2}\right)}{\prod_{j=1}^{6} G\left(u_{j} \pm z ; \omega_{1}, \omega_{2}\right)} d z .
$$

It follows from the asymptotics (4.4) of the hyperbolic gamma function that the condition (4.16) on the parameters ensures the absolute convergence of $E_{h}(u)$. Furthermore, $E_{h}(u)$ admits a unique meromorphic continuation to parameters $u \in \mathbb{C}^{6}$ satisfying (4.16) (in fact, it will be shown later that $E_{h}(u)$ has a unique meromorphic continuation to $u \in \mathbb{C}^{6}$ by relating $E_{h}$ to the hyperbolic Barnes integral $\left.B_{h}\right)$. Observe furthermore that $E_{h}(u)$ reduces to the hyperbolic beta integral (4.6) when the parameters $u \in \mathbb{C}^{6}$ satisfy the balancing condition $\sum_{j=1}^{6} u_{j}=4 i \omega$. We call $E_{h}(u)$ the hyperbolic Euler integral since its trigonometric analogue is a natural generalization of the Euler integral representation of the Gauss hypergeometric function, see Subsect. 5.4 and [5, §6.3].

Proposition 4.8. For $u \in \mathcal{G}_{2 i \omega}$ satisfying $\mathfrak{s}\left(u_{j}-i \omega\right)<0(j=1, \ldots, 8), \mathfrak{s}\left(\left(u_{7}+\right.\right.$ $\left.\left.u_{8}\right) / \omega_{1} \omega_{2}\right) \geq 0$ and (4.16), we have

$$
\lim _{r \rightarrow \infty} S_{h}\left(u-r \alpha_{78}^{-}\right) \exp \left(-\frac{\pi i}{\omega_{1} \omega_{2}}\left(u_{7}+u_{8}\right)\left(2 r-u_{7}+u_{8}\right)\right)=E_{h}\left(u_{1}, u_{2}, u_{3}, u_{4}, u_{5}, u_{6}\right) .
$$

Remark 4.9. Proposition 4.8 is trivial when $u_{7}=-u_{8}$ due to the reflection equation for $G$. The resulting limit is the hyperbolic beta integral (4.6) (since the balancing condition reduces to $\left.\sum_{j=1}^{6} u_{j}=4 i \omega\right)$. 
Proof. The assumptions on the parameters ensure that the integration contours in $S_{h}$ and $E_{h}$ can be chosen as the real line. We denote the integrand of the Euler integral by

$$
J(z)=\frac{G(i w \pm 2 z)}{\prod_{j=1}^{6} G\left(u_{j} \pm z\right)}
$$

and we set

$$
H(z)=\frac{G\left(z-u_{7}\right)}{G\left(z+u_{8}\right)} \exp \left(-\frac{\pi i z}{\omega_{1} \omega_{2}}\left(u_{7}+u_{8}\right)\right) .
$$

This allows us to write

$$
I_{h}\left(u-r \alpha_{78}^{-} ; z\right) \exp \left(-\frac{2 \pi i r}{\omega_{1} \omega_{2}}\left(u_{7}+u_{8}\right)\right)=J(z) H(r+z) H(r-z),
$$

where (recall) $I_{h}(u ; z)$ is the integrand of the hyperbolic hypergeometric function $S_{h}(u)$. Observe that $H$ is a continuous function on $\mathbb{R}$ satisfying

$$
\begin{aligned}
\lim _{z \rightarrow \infty} H(z) & =\exp \left(\frac{\pi i}{2 \omega_{1} \omega_{2}}\left(u_{8}^{2}-u_{7}^{2}\right)\right), \\
\lim _{z \rightarrow-\infty} H(z) \exp \left(\frac{2 \pi i z}{\omega_{1} \omega_{2}}\left(u_{7}+u_{8}\right)\right) & =\exp \left(\frac{\pi i}{2 \omega_{1} \omega_{2}}\left(u_{7}^{2}-u_{8}^{2}\right)\right)
\end{aligned}
$$

by (4.4) and by the reflection equation for the hyperbolic gamma function. Moreover, $H$ is uniformly bounded on $\mathbb{R}$ in view of the parameter condition $\Im\left(u_{7}+u_{8} / \omega_{1} \omega_{2}\right) \geq 0$ on the parameters, and we have

$$
\lim _{r \rightarrow \infty} H(r+z) H(r-z)=\exp \left(\frac{\pi i}{\omega_{1} \omega_{2}}\left(u_{8}^{2}-u_{7}^{2}\right)\right)
$$

for fixed $z \in \mathbb{R}$.

By Lebesgue's theorem of dominated convergence we conclude that

$$
\begin{aligned}
\lim _{r \rightarrow \infty} S_{h}\left(u-r \alpha_{78}^{-}\right) \exp \left(-\frac{2 \pi i r}{\omega_{1} \omega_{2}}\left(u_{7}+u_{8}\right)\right) & =\lim _{r \rightarrow \infty} \int_{\mathbb{R}} J(z) H(r+z) H(r-z) d z \\
& =\int_{\mathbb{R}} J(z) \lim _{r \rightarrow \infty} H(r+z) H(r-z) d z \\
& =E_{h}\left(u_{1}, \ldots, u_{6}\right) \exp \left(\frac{\pi i}{\omega_{1} \omega_{2}}\left(u_{8}^{2}-u_{7}^{2}\right)\right),
\end{aligned}
$$

as desired.

As a corollary of Proposition 4.8 we obtain the hyperbolic beta integral of AskeyWilson type, initially independently proved in [29] and in [37].

Corollary 4.10. For generic $u=\left(u_{1}, u_{2}, u_{3}, u_{4}\right) \in \mathbb{C}^{4}$ satisfying $\Im\left(\frac{1}{\omega_{1} \omega_{2}} \sum_{j=1}^{4} u_{j}\right)>$ $\Re\left(\frac{2 \omega}{\omega_{1} \omega_{2}}\right)$ we have

$$
\begin{aligned}
& \int_{\mathcal{C}} \frac{G(i \omega \pm 2 z)}{\prod_{j=1}^{4} G\left(u_{j} \pm z\right)} d z= 2 \sqrt{\omega_{1} \omega_{2}} G\left(u_{1}+u_{2}+u_{3}+u_{4}-3 i \omega\right) \\
& \times \prod_{1 \leq j<k \leq 4} G\left(i \omega-u_{j}-u_{k}\right) .
\end{aligned}
$$


Proof. Apply Proposition 4.8 under the additional condition $u_{5}=-u_{6}$ on the associated parameters $u \in \mathcal{G}_{2 i \omega}$. Using the reflection equation for the hyperbolic gamma function we see that the right-hand side of (4.17) becomes the hyperbolic Askey-Wilson integral. On the other hand, $S_{h}\left(u-r \alpha_{78}^{-}\right)$can be evaluated by the hyperbolic beta integral (4.6), resulting in

$$
\begin{aligned}
\int_{\mathcal{C}} \frac{G(i \omega \pm 2 z)}{\prod_{j=1}^{4} G\left(u_{j} \pm z\right)} d z= & 2 \sqrt{\omega_{1} \omega_{2}} G\left(i \omega-u_{7}-u_{8}\right) \prod_{1 \leq j<k \leq 4} G\left(i \omega-u_{j}-u_{k}\right) \\
& \times \lim _{r \rightarrow \infty} \exp \left(-\frac{\pi i}{\omega_{1} \omega_{2}}\left(u_{7}+u_{8}\right)\left(2 r-u_{7}+u_{8}\right)\right) \\
& \times \prod_{j=1}^{4} \frac{G\left(i \omega-u_{j}-u_{7}+r\right)}{G\left(-i \omega+u_{j}+u_{8}+r\right)} \\
= & 2 \sqrt{\omega_{1} \omega_{2}} G\left(u_{1}+u_{2}+u_{3}+u_{4}-3 i \omega\right) \prod_{1 \leq j<k \leq 4} G\left(i \omega-u_{j}-u_{k}\right),
\end{aligned}
$$

where we used the balancing condition on $u$ and the asymptotics (4.4) of the hyperbolic gamma function to obtain the last equality. The additional parameter restrictions which we have imposed in order to be able to apply Proposition 4.8 can now be removed by analytic continuation.

Since both the Euler and Barnes integrals are limits of the hyperbolic hypergeometric function we can connect them according to the following theorem.

Theorem 4.11. We have

$$
\begin{aligned}
B_{h}(u)= & E_{h}\left(u_{2}-s, u_{7}-s, u_{8}-s, u_{3}+s, u_{4}+s, u_{5}+s\right) \\
& \times \prod_{j=3}^{5} G\left(i \omega-u_{1}-u_{j}\right) \prod_{j=2,7,8} G\left(i \omega-u_{6}-u_{j}\right)
\end{aligned}
$$

as meromorphic functions in $\left\{u \in \mathcal{G}_{2 i \omega} \mid \mathfrak{\Im}\left(\left(u_{1}+u_{6}\right) / \omega_{1} \omega_{2}\right)<\Re\left(2 \omega / \omega_{1} \omega_{2}\right)\right\}$, where

$$
s=\frac{1}{2}\left(u_{2}+u_{6}+u_{7}+u_{8}\right)-i \omega=i \omega-\frac{1}{2}\left(u_{1}+u_{3}+u_{4}+u_{5}\right) .
$$

Proof. This theorem can be proved by degenerating a suitable $E_{7}$-symmetry of $S_{h}$ using Proposition 4.5 and Proposition 4.8. We prove the theorem here directly by analyzing the double integral

$$
\frac{1}{\sqrt{\omega_{1} \omega_{2}}} \int_{\mathbb{R}^{2}} \frac{G(i \omega \pm 2 z) \prod_{j=3}^{5} G\left(x-u_{j}\right)}{G(i \omega+s+x \pm z) G\left(x+u_{1}\right) \prod_{j=2,7,8} G\left(u_{j}-s \pm z\right)} d z d x
$$

for $\Re\left(\omega_{1}\right), \Re\left(\omega_{2}\right)>0, u \in \mathcal{G}_{2 i \omega}$ and $s=\frac{1}{2}\left(u_{2}+u_{6}+u_{7}+u_{8}\right)-i \omega$, where we impose the additional parameter restraints $\omega_{1} \omega_{2} \in \mathbb{R}_{>0}$ and

$$
|\Im(s)|<\Re(\omega), \quad \Im\left(u_{6}+s\right)<0
$$


to ensure absolute convergence of the double integral (which follows from a straightforward analysis of the integrand using (4.4) and (4.5), cf. the proof of Theorem 4.1), and

$\Im(s)<0, \quad \Im\left(i \omega-u_{j}\right)>0 \quad(j=1,3,4,5), \quad \Im\left(i \omega-u_{k}+s\right)>0 \quad(k=2,7,8)$

to ensure pole sequence separation by the integration contours. Note that these parameter restraints imply the parameter condition $\mathfrak{s}\left(u_{1}+u_{6}\right)<2 \Re(\omega)$ needed for the hyperbolic Euler integral in the right-hand side of (4.19) to be defined. Integrating the double integral first over $x$ and using the integral evaluation formula (4.13) of Barnes type, we obtain an expression of the double integral as a multiple of $E_{h}\left(u_{2}-s, u_{3}+s, u_{4}+\right.$ $\left.s, u_{5}+s, u_{7}-s, u_{8}-s\right)$. Integrating first over $z$ and using the hyperbolic Askey-Wilson integral (4.18), we obtain an expression of the double integral as a multiple of $B_{h}(u)$. The resulting identity is (4.19) for a restricted parameter domain. Analytic continuation now completes the proof.

Corollary 4.12. The hyperbolic Euler integral $E_{h}(u)$ has a unique meromorphic continuation to $u \in \mathbb{C}^{6}$ (which we also denote by $E_{h}(u)$ ).

From the degeneration from $S_{h}$ to $E_{h}$ (see Proposition 4.8) it is natural to interpret the parameter domain $\mathbb{C}^{6}$ as $\mathcal{G}_{2 i \omega} / \mathbb{C} \alpha_{78}^{-}$via the bijection

$$
\mathbb{C}^{6} \ni u \mapsto\left(u_{1}, \ldots, u_{6}, 2 i \omega-\sum_{j=1}^{6} u_{j}, 0\right)+\mathbb{C} \alpha_{78}^{-}
$$

We use this identification to transfer the natural $W_{1}\left(D_{6}\right)=W\left(E_{7}\right)_{\alpha_{78}^{-}}$-action on $\mathcal{G}_{2 i \omega} /$ $\mathbb{C} \alpha_{78}^{-}$to the parameter space $\mathbb{C}^{6}$ of the hyperbolic Euler integral. It is generated by permutations of $\left(u_{1}, \ldots, u_{6}\right)$ and by the action of $w \in W_{1}\left(D_{6}\right)$, which is given explicitly by

$$
w(u)=\left(u_{1}+s, u_{2}+s, u_{3}+s, u_{4}+s, u_{5}-s, u_{6}-s\right), \quad u \in \mathbb{C}^{6},
$$

where $s=i \omega-\frac{1}{2}\left(u_{1}+u_{2}+u_{3}+u_{4}\right)$. An interesting feature of $W_{1}\left(D_{6}\right)$-symmetries of the hyperbolic Euler integral (to be derived in Corollary 4.14), is the fact that the nontrivial $w$-symmetry of $E_{h}$ generalizes to the following explicit integral transformation for $E_{h}$.

Proposition 4.13. For periods $\omega_{1}, \omega_{2} \in \mathbb{C}$ with $\mathfrak{R}\left(\omega_{1}\right), \mathfrak{R}\left(\omega_{2}\right)>0$ and $\omega_{1} \omega_{2} \notin \mathbb{R}_{>0}$ and for parameters $s \in \mathbb{C}$ and $u=\left(u_{1}, \ldots, u_{6}\right) \in \mathbb{C}^{6}$ satisfying

$$
\mathfrak{s}\left(\frac{s}{\omega_{1} \omega_{2}}\right)>-\Re\left(\frac{\omega}{\omega_{1} \omega_{2}}\right), \quad \mathfrak{s}\left(\frac{u_{1}+u_{2}+u_{3}+u_{4}}{\omega_{1} \omega_{2}}\right), \Im\left(\frac{u_{5}+u_{6}-2 s}{\omega_{1} \omega_{2}}\right)>\Re\left(\frac{2 \omega}{\omega_{1} \omega_{2}}\right)
$$

and

$$
\Im\left(u_{j}-i \omega\right)<0 \quad(j=1, \ldots, 4), \quad \Im\left(u_{k}-i \omega\right)<\Im(s)<0 \quad(k=5,6),
$$

we have

$$
\begin{aligned}
& \int_{\mathbb{R}} E_{h}\left(u_{1}, u_{2}, u_{3}, u_{4}, i \omega+s+x, i \omega+s-x\right) \frac{G(i \omega \pm 2 x)}{G\left(u_{5}-s \pm x, u_{6}-s \pm x\right)} d x \\
& \quad=2 \sqrt{\omega_{1} \omega_{2}} \frac{G\left(i \omega-u_{5}-u_{6}+2 s\right)}{G\left(i \omega-u_{5}-u_{6}, i \omega+2 s\right)} E_{h}(u) .
\end{aligned}
$$


Proof. Observe that the requirement $\omega_{1} \omega_{2} \notin \mathbb{R}_{>0}$ ensures the existence of parameters $u \in \mathbb{C}^{6}$ and $s \in \mathbb{C}$ satisfying the restraints (4.22) and (4.23). Furthermore, (4.22) ensures that

$$
\Im\left(\frac{1}{\omega_{1} \omega_{2}}\left(\sum_{j=1}^{6} u_{j}\right)\right), \Im\left(\frac{1}{\omega_{1} \omega_{2}}\left(\sum_{j=1}^{4} u_{j}+2 i \omega+2 s\right)\right)>\mathfrak{i}\left(\frac{2 \omega}{\omega_{1} \omega_{2}}\right),
$$

hence both hyperbolic Euler integrals in (4.24) are defined. We derive the integral transformation (4.24) by considering the double integral

$$
\int_{\mathbb{R}^{2}} \frac{G(i \omega \pm 2 z, i \omega \pm 2 x)}{G(i \omega+s \pm x \pm z) \prod_{j=1}^{4} G\left(u_{j} \pm z\right) \prod_{k=5}^{6} G\left(u_{k}-s \pm x\right)} d z d x,
$$

which absolutely converges by (4.22). Integrating the double integral first over $x$ using the hyperbolic Askey-Wilson integral (4.18) yields the right-hand side of (4.24). Integrating first over $z$ results in the left-hand side of (4.24).

Corollary 4.14. The hyperbolic Euler integral $E_{h}(u)\left(u \in \mathbb{C}^{6}\right)$ is symmetric in $\left(u_{1}, \ldots, u_{6}\right)$ and it satisfies

$$
E_{h}(u)=E_{h}(w u) G\left(i \omega-u_{5}-u_{6}\right) G\left(\sum_{j=1}^{6} u_{j}-3 i \omega\right) \prod_{1 \leq j<k \leq 4} G\left(i \omega-u_{j}-u_{k}\right)
$$

as meromorphic functions in $u \in \mathbb{C}^{6}$.

Proof. The permutation symmetry is trivial. For (4.25) we apply Proposition 4.13 with $s=i \omega-\frac{1}{2}\left(u_{1}+u_{2}+u_{3}+u_{4}\right)$. The hyperbolic Euler integral in the left-hand side of the integral transformation (4.24) can now be evaluated by the hyperbolic beta integral (4.6). The remaining integral is an explicit multiple of $E_{h}(w u)$. The resulting identity yields (4.25) for a restricted parameter domain. Analytic continuation completes the proof.

Remark 4.15. The $w$-symmetry (4.25) of $E_{h}$ can also be proved by degenerating the $w$-symmetry of $S_{h}$, or by relating (4.25) to a $W_{2}\left(D_{6}\right)$-symmetry of $B_{h}$ using Theorem 4.11.

The longest Weyl group element $v_{1} \in W_{1}\left(D_{6}\right)$ and the longest Weyl group element $v \in W\left(E_{7}\right)$ have the same action on $\mathcal{G}_{2 i \omega} / \mathbb{C} \alpha_{78}^{-}$. Consequently, under the identification (4.20), $v_{1}$ acts on $\mathbb{C}^{6}$ by

$$
v_{1}(u)=\left(i \omega-u_{1}, \ldots, i \omega-u_{6}\right), \quad u \in \mathbb{C}^{6} .
$$

Corollary 4.16. The symmetry of the hyperbolic Euler integral $E_{h}(u)$ with respect to the longest Weyl group element $v_{1} \in W_{1}\left(D_{6}\right)$ is

$$
E_{h}(u)=E_{h}\left(v_{1} u\right) G\left(-3 i \omega+\sum_{j=1}^{6} u_{j}\right) \prod_{1 \leq j<k \leq 6} G\left(i \omega-u_{j}-u_{k}\right)
$$

as meromorphic functions in $u \in \mathbb{C}^{6}$. 
Proof. For parameters $u \in \mathcal{G}_{2 i \omega}$ such that both $u$ and $v u$ satisfy the parameter restraints of Proposition 4.8, we degenerate the $v$-symmetry (4.9) of $S_{h}$ using (4.17). Analytic continuation completes the proof.

The contiguous relations for $S_{h}$ degenerate to the following contiguous relations for $E_{h}$.

Lemma 4.17. We have

$$
\begin{aligned}
& \frac{\prod_{j=1}^{4} s\left(\left(u_{j}+u_{5}\right) / \omega_{2}\right)}{s\left(\left(u_{5}-u_{6}+2 i \omega\right) / \omega_{2}\right)}\left(E_{h}\left(\tau_{65}^{i \omega_{1}} u\right)-E_{h}(u)\right)-\left(u_{5} \leftrightarrow u_{6}\right) \\
& =s\left(\left(u_{5} \pm u_{6}\right) / \omega_{2}\right) s\left(\left(2 i \omega-\sum_{j=1}^{6} u_{j}\right) / \omega_{2}\right) E_{h}(u)
\end{aligned}
$$

as meromorphic functions in $u \in \mathbb{C}^{6}$.

Proof. Use Proposition 4.8 to degenerate the contiguous relation (4.11) for the hyperbolic hypergeometric function $S_{h}$ to $E_{h}$.

4.6. Ruijsenaars' R-function. Motivated by the theory of quantum integrable, relativistic particle systems on the line, Ruijsenaars [26, 28, 29] introduced and studied a generalized hypergeometric $R$-function $R$, which is essentially the hyperbolic Barnes integral $B_{h}(u)$ with respect to a suitable reparametrization (and re-interpretation) of the parameters $u \in \mathcal{G}_{2 i \omega}$. The new parameters will be denoted by $(\gamma, x, \lambda) \in \mathbb{C}^{6}$ with $\gamma=\left(\gamma_{1}, \ldots, \gamma_{4}\right)^{T} \in \mathbb{C}^{4}$, where $x$ (respectively $\lambda$ ) is viewed as the geometric (respectively spectral) parameter, while the four parameters $\gamma_{j}$ are viewed as coupling constants. As a consequence of the results derived in the previous subsections, we will re-derive many of the properties of the generalized hypergeometric $R$-function, and we obtain a new integral representation of $R$ in terms of the hyperbolic Euler integral $E_{h}$.

Set

$$
N(\gamma)=\prod_{j=1}^{3} G\left(i \gamma_{0}+i \gamma_{j}+i \omega\right)
$$

Ruijsenaars' [26] generalized hypergeometric function $R\left(\gamma ; x, \lambda ; \omega_{1}, \omega_{2}\right)=R(\gamma, \lambda)$ is defined by

$$
R(\gamma ; x, \lambda)=\frac{1}{2 \sqrt{\omega_{1} \omega_{2}}} \frac{N(\gamma)}{G\left(i \gamma_{0} \pm x, i \hat{\gamma}_{0} \pm \lambda\right)} B_{h}(u),
$$

where $u \in \mathcal{G}_{2 i \omega} / \mathbb{C} \beta_{1278}$ with

$$
\begin{gathered}
u_{1}=i \omega, \quad u_{2}=i \omega+i \gamma_{0}+i \gamma_{1}, \quad u_{3}=-i \gamma_{0}+x, \quad u_{4}=-i \gamma_{0}-x, \\
u_{5}=-i \hat{\gamma}_{0}+\lambda, \quad u_{6}=-i \hat{\gamma}_{0}-\lambda, \quad u_{7}=i \omega+i \gamma_{0}+i \gamma_{2}, \quad u_{8}=i \omega+i \gamma_{0}+i \gamma_{3} .
\end{gathered}
$$

Note that $R\left(\gamma ; x, \lambda ; \omega_{1}, \omega_{2}\right)$ is invariant under permuting the role of the two periods $\omega_{1}$ and $\omega_{2}$. Observe furthermore that the map $(\gamma, x, \lambda) \rightarrow u+\mathbb{C} \beta_{1278}$, with $u$ given by (4.28), defines a bijection $\mathbb{C}^{6} \stackrel{\sim}{\rightarrow} \mathcal{G}_{2 i \omega} / \mathbb{C} \beta_{1278}$. 
We define the dual parameters $\hat{\gamma}$ by

$$
\hat{\gamma}=\frac{1}{2}\left(\begin{array}{cccc}
1 & 1 & 1 & 1 \\
1 & 1 & -1 & -1 \\
1 & -1 & 1 & -1 \\
1 & -1 & -1 & 1
\end{array}\right) \gamma .
$$

We will need the following auxiliary function:

$$
c(\gamma ; y)=\frac{1}{G(2 y+i \omega)} \prod_{j=0}^{3} G\left(y-i \gamma_{j}\right) .
$$

The following proposition was derived by different methods in [28].

Proposition 4.18. $R$ is even in $x$ and $\lambda$ and self-dual, i.e.

$$
R(\gamma ; x, \lambda)=R(\gamma ;-x, \lambda)=R(\gamma ; x,-\lambda)=R(\hat{\gamma} ; \lambda, x) .
$$

Furthermore, for an element $\sigma \in W\left(D_{4}\right)$, where $W\left(D_{4}\right)$ is the Weyl-group of type $D_{4}$ acting on the parameters $\gamma$ by permutations and even numbers of sign fips, we have

$$
\frac{R(\gamma ; x, \lambda)}{c(\gamma ; x) c(\hat{\gamma} ; \lambda) N(\gamma)}=\frac{R(\sigma \gamma ; x, \lambda)}{c(\sigma \gamma ; x) c(\widehat{\sigma \gamma} ; \lambda) N(\sigma \gamma)} \text {. }
$$

Proof. These symmetries are all direct consequences of the $W_{2}\left(D_{6}\right)$-symmetries of the hyperbolic Barnes integral $B_{h}$ (see Proposition 4.7). Concretely, note that the $W_{2}\left(D_{6}\right)$ action on $\mathbb{C}^{6} \simeq \mathcal{G}_{2 i \omega} / \mathbb{C} \beta_{1278}$ is given by

$$
\begin{aligned}
s_{78}(\gamma, x, \lambda)= & \left(\gamma_{0}, \gamma_{1}, \gamma_{3}, \gamma_{2}, x, \lambda\right), \\
s_{18}(\gamma, x, \lambda)= & \left(-\gamma_{3}, \gamma_{1}, \gamma_{2},-\gamma_{0}, x, \lambda\right), \\
w(\gamma, x, \lambda)= & \left(\gamma_{1}, \gamma_{0}, \gamma_{2}, \gamma_{3}, x, \lambda\right), \\
s_{45}(\gamma, x, \lambda)= & \left(\frac{1}{2}\left(\gamma_{0}+\hat{\gamma}_{0}\right)+\frac{i}{2}(x+\lambda), \frac{1}{2}\left(\gamma_{1}+\hat{\gamma}_{1}\right)-\frac{i}{2}(x+\lambda), \frac{1}{2}\left(\gamma_{2}+\hat{\gamma}_{2}\right)-\frac{i}{2}(x+\lambda),\right. \\
& \left.\frac{1}{2}\left(\gamma_{3}+\hat{\gamma}_{3}\right)-\frac{i}{2}(x+\lambda), \frac{i}{2}\left(\hat{\gamma}_{0}-\gamma_{0}\right)+\frac{1}{2}(x-\lambda), \frac{i}{2}\left(\hat{\gamma}_{0}-\gamma_{0}\right)+\frac{1}{2}(\lambda-x)\right), \\
s_{34}(\gamma, x, \lambda)= & (\gamma,-x, \lambda), \\
s_{56}(\gamma, x, \lambda)= & (\gamma, x,-\lambda) .
\end{aligned}
$$

The fact that $R(\gamma ; x, \lambda)$ is even in $x$ and $\lambda$ follows now from the $s_{34} \in W_{2}\left(D_{6}\right)$ and $s_{56} \in W_{2}\left(D_{6}\right)$ symmetry of $B_{h}$, respectively (see Proposition 4.7). Similarly, the duality is obtained from the action of $s_{35} s_{46}$ and using that $\gamma_{0}+\gamma_{i}=\hat{\gamma}_{0}+\hat{\gamma}_{i}(i=1,2,3)$, while the $W\left(D_{4}\right)$-symmetry in $\gamma$ follows from considering the action of $s_{27} \in W_{2}\left(D_{6}\right)$ (which interchanges $\left.\gamma_{1} \leftrightarrow \gamma_{2}\right), s_{78}, s_{18}$ and $w$.

Remark 4.19. Corollary 4.6 implies the explicit evaluation formula

$$
R\left(\gamma ; i \omega+i \gamma_{3}, \lambda ; \omega_{1}, \omega_{2}\right)=\prod_{j=1}^{2} \frac{G\left(i \omega+i \gamma_{0}+i \gamma_{j}\right)}{G\left(i \omega+i \gamma_{j}+i \gamma_{3}\right) G\left(i \hat{\gamma}_{j} \pm \lambda\right)}
$$

Using the $W\left(D_{4}\right)$-symmetry of $R$, this implies

$$
R\left(\gamma ; i \omega+i \gamma_{0}, \lambda ; \omega_{1}, \omega_{2}\right)=1,
$$

in accordance with [26, (3.26)]. 
Using Proposition 4.7 and Theorem 4.11 we can derive several different integral representations of the $R$-function. First we derive the integral representation of $R$ which was previously derived in [1] by relating $R$ to matrix coefficients of representations of the modular double of the quantum group $\mathcal{U}_{q}\left(\mathfrak{s l}_{2}(\mathbb{C})\right)$.

Proposition 4.20. We have

$$
R(\gamma ; x, \lambda)=\frac{N(\gamma)}{2 \sqrt{\omega_{1} \omega_{2}}} \frac{G\left(x-i \gamma_{0}, x-i \gamma_{1}, \lambda-i \hat{\gamma}_{0}, \lambda-i \hat{\gamma}_{1}\right)}{G\left(x+i \gamma_{2}, x+i \gamma_{3}, \lambda+i \hat{\gamma}_{2}, \lambda+i \hat{\gamma}_{3}\right)} B_{h}(v),
$$

where

$$
\begin{array}{ll}
v_{1 / 2}=x-\frac{\lambda}{2}+\frac{i \omega}{2} \pm \frac{i}{2}\left(\gamma_{0}-\gamma_{1}\right), & v_{3 / 4}=-x-\frac{\lambda}{2}+\frac{i \omega}{2} \pm \frac{i}{2}\left(\gamma_{3}-\gamma_{2}\right), \\
v_{5 / 6}=\frac{\lambda}{2}+\frac{i \omega}{2} \pm \frac{i}{2}\left(-\gamma_{0}-\gamma_{1}\right), & v_{7 / 8}=\frac{\lambda}{2}+\frac{i \omega}{2} \pm \frac{i}{2}\left(\gamma_{2}+\gamma_{3}\right),
\end{array}
$$

and $v_{j / k}=\alpha \pm \beta$ means $v_{j}=\alpha+\beta$ and $v_{k}=\alpha-\beta$.

Proof. Express $B_{h}\left(s_{36} w s_{35} s_{28} w s_{18} u\right)$ in terms of $B_{h}(u)$ using the $W_{2}\left(D_{6}\right)$-symmetries of the hyperbolic Barnes integral $B_{h}$ (see Proposition 4.7) and specialize $u$ as in (4.28). This gives the desired equality.

Moreover we can express $R$ in terms of the hyperbolic Euler integral $E_{h}$, which leads to a previously unknown integral representation.

Theorem 4.21. We have

$$
\begin{aligned}
R(\gamma ; x, \lambda) & =\frac{1}{2 \sqrt{\omega_{1} \omega_{2}}} \frac{\prod_{j=1}^{3} G\left(i \gamma_{0}+i \gamma_{j}+i \omega, \lambda-i \hat{\gamma}_{j}\right)}{G\left(\lambda+i \hat{\gamma}_{0}\right)} E_{h}(u) \\
& =\frac{1}{2 \sqrt{\omega_{1} \omega_{2}}} \frac{\prod_{j=1}^{3} G\left(i \gamma_{0}+i \gamma_{j}+i \omega, \lambda-i \hat{\gamma}_{j}\right)}{G\left(\lambda+i \hat{\gamma}_{0}\right) \prod_{j=0}^{3} G\left(i \gamma_{j} \pm x\right)} E_{h}(v),
\end{aligned}
$$

where $u \in \mathbb{C}^{6}$ is given by

$$
u_{j}=\frac{i \omega}{2}+i \gamma_{j-1}-\frac{i \hat{\gamma}_{0}}{2}+\frac{\lambda}{2}, \quad(j=1, \ldots, 4), \quad u_{5 / 6}=\frac{i \omega}{2} \pm x+\frac{i \hat{\gamma}_{0}}{2}-\frac{\lambda}{2},
$$

and $v \in \mathbb{C}^{6}$ is given by

$$
v_{j}=\frac{i \omega}{2}-i \gamma_{j-1}+\frac{i \hat{\gamma}_{0}}{2}+\frac{\lambda}{2}, \quad(j=1, \ldots, 4), \quad v_{5 / 6}=\frac{i \omega}{2} \pm x-\frac{i \hat{\gamma}_{0}}{2}-\frac{\lambda}{2}
$$

Proof. To prove the first equation, express $R(\gamma ; x, \lambda)$ in terms of $R\left(-\gamma_{3}, \gamma_{1}, \gamma_{2},-\gamma_{0}\right.$; $x, \lambda)$ using the $W\left(D_{4}\right)$-symmetry of $R$ (see Proposition 4.18). Subsequently use the identity relating $B_{h}$ to $E_{h}$, see Theorem 4.11. To obtain the second equation, apply the symmetry of $E_{h}$ with respect to the longest Weyl-group element $v_{1} \in W_{1}\left(D_{6}\right)$ (see Corollary 4.16) in the first equation and use that $R$ is even in $\lambda$.

The contiguous relation for $E_{h}$ (Lemma 4.17) now becomes the following result. 
Proposition 4.22. ([26]) Ruijsenaars' R-function satisfies the Askey-Wilson second order difference equation

$$
\begin{aligned}
& A\left(\gamma ; x ; \omega_{1}, \omega_{2}\right)\left(R\left(\gamma ; x+i \omega_{1}, \lambda\right)-R(\gamma ; x, \lambda)\right)+(x \leftrightarrow-x) \\
& \quad=B\left(\gamma ; \lambda ; \omega_{1}, \omega_{2}\right) R(\gamma ; x, \lambda),
\end{aligned}
$$

where

$$
\begin{aligned}
& A\left(\gamma ; x ; \omega_{1}, \omega_{2}\right)=\frac{\prod_{j=0}^{3} s\left(\left(i \omega+x+i \gamma_{j}\right) / \omega_{2}\right)}{s\left(2 x / \omega_{2}\right) s\left(2(x+i \omega) / \omega_{2}\right)}, \\
& B\left(\gamma ; \lambda ; \omega_{1}, \omega_{2}\right)=s\left(\left(\lambda-i \omega-i \hat{\gamma}_{0}\right) / \omega_{2}\right) s\left(\left(\lambda+i \omega+i \hat{\gamma}_{0}\right) / \omega_{2}\right) .
\end{aligned}
$$

Remark 4.23. As is emphasized in [26], $R$ satisfies four Askey-Wilson second order difference equations; two equations acting on the geometric variable $x$ (namely (4.30), and (4.30) with the role of $\omega_{1}$ and $\omega_{2}$ interchanged), as well as two equations acting on the spectral parameter $\lambda$ by exploring the duality of $R$ (see Proposition 4.18).

For later purposes, it is convenient to rewrite (4.30) as the eigenvalue equation

$$
\left(\mathcal{L}_{\gamma}^{\omega_{1}, \omega_{2}} R\left(\gamma ; \cdot, \lambda ; \omega_{1}, \omega_{2}\right)\right)(x)=B\left(\gamma ; \lambda ; \omega_{1}, \omega_{2}\right) R\left(\gamma ; x, \lambda ; \omega_{1}, \omega_{2}\right)
$$

for the Askey-Wilson second order difference operator

$$
\left(\mathcal{L}_{\gamma}^{\omega, \omega_{2}} f\right)(x):=A\left(\gamma ; x ; \omega_{1}, \omega_{2}\right)\left(f\left(x+i \omega_{1}\right)-f(x)\right)+(x \leftrightarrow-x) .
$$

\section{Trigonometric Hypergeometric Integrals}

5.1. Basic hypergeometric series. In this section we assume that the base $q$ satisfies $0<|q|<1$. The trigonometric gamma function [24] is essentially the $q$-gamma function $\Gamma_{q}(x)$, see [5]. For ease of presentation we express all the results in terms of the $q$-shifted factorial $(z ; q)_{\infty}$, which are related to $\Gamma_{q}(x)$ by

$$
\Gamma_{q}(x)=\frac{(q ; q)_{\infty}}{\left(q^{x} ; q\right)_{\infty}}(1-q)^{1-x}
$$

(with a proper interpretation of the right-hand side). The $q$-shifted factorial is the $p=0$ degeneration of the elliptic gamma function,

$$
\Gamma_{e}(z ; 0, q)=\frac{1}{(z ; q)_{\infty}},
$$

while the role of the first order analytic difference equation is taken over by

$$
(z ; q)_{\infty}=(1-z)(q z ; q)_{\infty}
$$

However there is no reflection equation anymore; its role is taken over by the product formula for Jacobi's (renormalized) theta function

$$
\theta(z ; q)=(z, q / z ; q)_{\infty}
$$

As a function of $z$ the $q$-shifted factorial $(z ; q)_{\infty}$ is entire with zeros at $z=q^{-n}$ for $n \in \mathbb{Z}_{\geq 0}$. In this section we call a sequence of the form $a q^{-n}\left(n \in \mathbb{Z}_{\geq 0}\right)$ an upward 
sequence (since they diverge to infinity for large $n$ ) and a sequence of the form $a q^{n}$ ( $n \in \mathbb{Z}_{\geq 0}$ ) a downward sequence (as the elements converge to zero for large $n$ ).

We will use standard notations for basic hypergeometric series from [5]. In particular, the ${ }_{r+1} \phi_{r}$ basic hypergeometric series is

$$
{ }_{r+1} \phi_{r}\left(\begin{array}{c}
a_{1}, \ldots, a_{r+1} \\
b_{1}, \ldots b_{r}
\end{array} ; q, z\right)=\sum_{n=0}^{\infty} \frac{\left(a_{1}, \ldots, a_{r+1} ; q\right)_{n}}{\left(q, b_{1}, \ldots, b_{r} ; q\right)_{n}} z^{n}, \quad|z|<1,
$$

where $(a ; q)_{n}=\prod_{j=0}^{n-1}\left(1-a q^{j}\right)$ and with the usual convention regarding products of such expressions. The very-well-poised ${ }_{r+1} \phi_{r}$ basic hypergeometric series is

$$
{ }_{r+1} W_{r}\left(a_{1} ; a_{4}, a_{5}, \ldots, a_{r+1} ; q, z\right)={ }_{r+1} \phi_{r}\left(\begin{array}{c}
a_{1}, q a_{1}^{\frac{1}{2}},-q a_{1}^{\frac{1}{2}}, a_{4}, \ldots, a_{r+1} ; q, z \\
a_{1}^{\frac{1}{2}},-a_{1}^{\frac{1}{2}}, q a_{1} / a_{4}, \ldots, q a_{1} / a_{r+1}
\end{array}\right) \text {. }
$$

Finally, the bilateral basic hypergeometric series ${ }_{r} \psi_{r}$ is defined as

$$
\begin{aligned}
{ }_{r} \psi_{r}\left(\begin{array}{l}
a_{1}, a_{2}, \ldots, a_{r} \\
b_{1}, b_{2}, \ldots, b_{r}
\end{array} ;, z\right)= & \sum_{n=0}^{\infty} \frac{\left(a_{1}, a_{2}, \ldots, a_{r} ; q\right)_{n}}{\left(b_{1}, b_{2}, \ldots, b_{r} ; q\right)_{n}} z^{n} \\
& +\sum_{n=1}^{\infty} \frac{\left(q / b_{1}, q / b_{2}, \ldots, q / b_{r} ; q\right)_{n}}{\left(q / a_{1}, q / a_{2}, \ldots, q / a_{r} ; q\right)_{n}}\left(\frac{b_{1} \cdots b_{r}}{a_{1} \cdots a_{r} z}\right)^{n},
\end{aligned}
$$

provided that $\left|b_{1} \cdots b_{r} / a_{1} \cdots a_{r}\right|<|z|<1$ to ensure absolute and uniform convergence.

We end this introductory subsection by an elementary lemma which will enable us to rewrite trigonometric integrals with compact integration cycle in terms of trigonometric integrals with noncompact integration cycle. Let $\mathbb{H}_{+}$be the upper half plane in $\mathbb{C}$. In this section we choose $\tau \in \mathbb{H}_{+}$such that $q=e(\tau)$ once and for all, where $e(x)$ is a shorthand notation for $\exp (2 \pi i x)$. We furthermore write $\Lambda=\mathbb{Z}+\mathbb{Z} \tau$.

Lemma 5.1. Let $u, v \in \mathbb{C}$ be such that $u \notin v+\Lambda$. There exists an $\eta=\eta(u, v) \in \mathbb{C}$, unique up to $\Lambda$-translates, such that

$$
\begin{aligned}
& \frac{\theta(e(u+v-\eta-x), e(x-\eta) ; q)}{\theta(e(u-\eta), e(v-\eta) ; q)} \\
& =\frac{(e((v-u) / \tau)-1) \theta(e(x-u), e(v-x) ; q)}{\tau(q, q ; q)_{\infty} \theta(e(v-u) ; q)} \\
& \quad \times \sum_{n=-\infty}^{\infty} \frac{1}{(1-e((v-x-n) / \tau))(e((x+n-u) / \tau)-1)} .
\end{aligned}
$$

Proof. Set $\widetilde{q}=e(-1 / \tau)$. The bilateral sum

$$
\begin{aligned}
f(x) & =\sum_{n=-\infty}^{\infty} \frac{1}{(1-e((v-x-n) / \tau))(e((x+n-u) / \tau)-1)} \\
& =\frac{1}{(1-e((v-x) / \tau))(e((x-u) / \tau)-1)} 2 \psi_{2}\left(\begin{array}{c}
e((v-x) / \tau), e((u-x) / \tau) \\
\widetilde{q} e((v-x) / \tau), \widetilde{q} e((u-x) / \tau)
\end{array} \tilde{q}, \widetilde{q}\right)
\end{aligned}
$$


defines an elliptic function on $\mathbb{C} / \Lambda$, with possible poles at most simple and located at $u+\Lambda$ and at $v+\Lambda$. Hence there exists a $\eta \in \mathbb{C}$ (unique up to $\Lambda$-translates) and a constant $C_{\eta} \in \mathbb{C}$ such that

$$
f(x)=C_{\eta} \frac{\theta(e(u+v-\eta-x), e(x-\eta) ; q)}{\theta(e(x-u), e(v-x) ; q)} .
$$

We now compute the residue of $f$ at $u$ in two different ways:

$$
\operatorname{Res}_{x=u}(f)=\frac{\tau}{2 \pi i} \frac{1}{(1-e((v-u) / \tau))}
$$

from the bilateral series expression of $f$, and

$$
\operatorname{Res}_{x=u}(f)=-\frac{C_{\eta}}{2 \pi i} \frac{\theta(e(u-\eta), e(v-\eta) ; q)}{(q, q ; q)_{\infty} \theta(e(v-u) ; q)}
$$

from the expression of $f$ as a quotient of theta-functions. Combining both identities yields an explicit expression of the constant $C_{\eta}$ in terms of $\eta$, resulting in the formula

$$
f(x)=\frac{\tau(q, q ; q)_{\infty} \theta(e(v-u) ; q)}{(e((v-u) / \tau)-1)} \frac{\theta(e(u+v-\eta-x), e(x-\eta) ; q)}{\theta(e(x-u), e(v-x), e(u-\eta), e(v-\eta) ; q)}
$$

for $f$. Rewriting this identity yields the desired result.

5.2. Trigonometric hypergeometric integrals with $E_{6}$ symmetries. We consider trigonometric degenerations of $S_{e}(t)\left(t \in \mathcal{H}_{p q}\right)$ along root vectors $\alpha \in R\left(E_{8}\right)$ lying in the $W\left(E_{7}\right)=W\left(E_{8}\right)_{\delta}$-orbit

$$
\mathcal{O}:=W\left(E_{7}\right)\left(\alpha_{18}^{+}\right)=\left\{\alpha_{j k}^{+}, \gamma_{j k} \mid 1 \leq j<k \leq 8\right\},
$$

cf. Sect. 2. The degenerations relate to the explicit bijection

$$
\mathcal{G}_{0} \stackrel{\sim}{\longrightarrow} \mathcal{G}_{\log (p q)}, \quad\left(u_{1}, \ldots, u_{8}\right) \mapsto\left(u_{1}, \ldots, u_{8}\right)+\log (p q) \alpha
$$

on the parameter spaces (in logarithmic form) of the associated integrals. We obtain two different trigonometric degenerations, depending on whether we degenerate along an orbit vector of the form $\alpha=\alpha_{j k}^{+}$, or of the form $\gamma_{j k}$.

Specifically, we consider the trigonometric degenerations $S_{t}(t)$ respectively $U_{t}(t)$ ( $\left.t \in \mathcal{H}_{1}\right)$ of $S_{e}(t)\left(t \in \mathcal{H}_{p q}\right)$ along the orbit vector $\alpha_{18}^{+}$and $\gamma_{18}$ respectively. The orbit vector $\alpha_{18}^{+}$(respectively $\gamma_{18}$ ) is the additional simple root turning the basis $\Delta_{1}$ (respectively $\Delta_{2}$ ) of $R\left(E_{7}\right)$ into the basis $\bar{\Delta}_{1}$ (respectively $\bar{\Delta}_{2}$ ) of $R\left(E_{8}\right)$, see Sect. 2. The induced symmetry group of $S_{t}(t)\left(t \in \mathcal{H}_{1}\right)$ is the isotropy subgroup $W\left(E_{7}\right)_{\alpha_{18}^{+}}$of $W\left(E_{7}\right)$, while the induced symmetry group of $U_{t}(t)\left(t \in \mathcal{H}_{1}\right)$ is $W\left(E_{7}\right)_{\gamma_{18}}$. It follows from the analysis in Sect. 2 that $W\left(E_{7}\right)_{\alpha_{18}^{+}}=W\left(E_{7}\right)_{\gamma_{18}}$ is a maximal, standard parabolic subgroup of $W\left(E_{7}\right)$ with respect to both bases $\Delta_{1}$ and $\Delta_{2}$, isomorphic to the Weyl group $W\left(E_{6}\right)$ of type $E_{6}$, with corresponding simple roots $\Delta_{1}^{\prime}=\Delta_{1} \backslash\left\{\alpha_{21}^{-}\right\}$and $\Delta_{2}^{\prime}=\Delta_{2} \backslash\left\{\alpha_{87}^{-}\right\}$, and with corresponding Dynkin diagrams, 


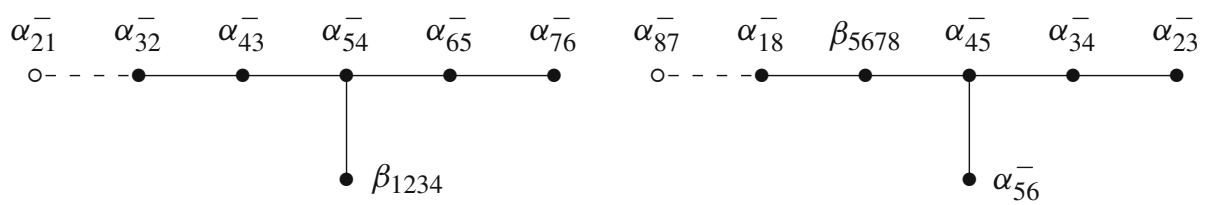

Observe that $\alpha_{18}^{-}$and $\alpha_{76}^{-}$are the highest roots of the standard parabolic root system $R\left(E_{6}\right)$ of type $E_{6}$ in $R\left(E_{7}\right)$ corresponding to the bases $\Delta_{1}^{\prime}$ and $\Delta_{2}^{\prime}$ respectively. From now on we write

$$
W\left(E_{6}\right):=W\left(E_{7}\right)_{\alpha_{18}^{+}}=W\left(E_{7}\right)_{\gamma_{18}} .
$$

We first introduce the trigonometric hypergeometric integrals $S_{t}(t)$ and $U_{t}(t)$ $\left(t \in \mathcal{H}_{1}\right)$ explicitly. Their integrands are defined by

$$
\begin{aligned}
I_{t}(t ; z) & =\frac{\left(z^{ \pm 2}, t_{1}^{-1} z^{ \pm 1}, t_{8}^{-1} z^{ \pm 1} ; q\right)_{\infty}}{\prod_{j=2}^{7}\left(t_{j} z^{ \pm 1} ; q\right)_{\infty}}, \\
J_{t}^{\mu}(t ; z) & =2 \frac{\theta\left(t_{1} t_{8} / \mu z, z / \mu ; q\right)}{\theta\left(t_{1} / \mu, t_{8} / \mu ; q\right)}\left(1-\frac{z^{2}}{q}\right) \prod_{i=2}^{7} \frac{\left(z / t_{i} ; q\right)_{\infty}}{\left(t_{i} z ; q\right)_{\infty}} \prod_{j=1,8} \frac{1}{\left(t_{j} z / q, t_{j} / z ; q\right)_{\infty}},
\end{aligned}
$$

where $t=\left(t_{1}, \ldots, t_{8}\right) \in\left(\mathbb{C}^{\times}\right)^{8}$. For generic $t=\left(t_{1}, \ldots, t_{8}\right) \in \mathbb{C}^{8}$ satisfying $\prod_{j=1}^{8} t_{j}=$ 1 and generic $\mu \in \mathbb{C}$ we now define the resulting trigonometric hypergeometric integrals as

$$
S_{t}(t)=\int_{\mathcal{C}} I_{t}(t ; z) \frac{d z}{2 \pi i z}, \quad U_{t}^{\mu}(t)=\int_{\mathcal{C}^{\prime}} J_{t}^{\mu}(t ; q) \frac{d z}{2 \pi i z},
$$

where $\mathcal{C}$ (respectively $\mathcal{C}^{\prime}$ ) is a deformation of the positively oriented unit circle $\mathbb{T}$ including the pole sequences $t_{j} q^{\mathbb{Z}_{\geq 0}}(j=2, \ldots, 7)$ of $I_{t}(t ; z)$ and excluding their reciprocals (respectively including the pole sequences $t_{j} q^{\mathbb{Z}_{\geq 0}}(j=1,8)$ of $J_{t}^{\mu}(t ; z)$ and excluding the pole sequences $t_{j}^{-1} q^{\mathbb{Z}_{\leq 1}}(j=1,8)$ and $\left.t_{i}^{-1} q^{\mathbb{Z}_{\leq 0}}(i=2, \ldots, 7)\right)$. As in the elliptic and hyperbolic cases, one observes that $S_{t}(t)$ (respectively $U_{t}^{\mu}(t)$ ) admits a unique meromorphic extension to the parameter domain $\left\{t \in \mathbb{C}^{8} \mid \prod_{j=1}^{8} t_{j}=1\right\}$ (respectively $\left.\left\{(\mu, t) \in \mathbb{C}^{\times} \times \mathbb{C}^{8} \mid \prod_{j=1}^{8} t_{j}=1\right\}\right)$. We call $S_{t}(t)$ the trigonometric hypergeometric function.

Lemma 5.2. The integral $U_{t}^{\mu}(t)$ is independent of $\mu \in \mathbb{C}^{\times}$.

Proof. There are several different, elementary arguments to prove the lemma; we give here the argument based on Liouville's Theorem. Note that $U_{t}^{q \mu}(t)=U_{t}^{\mu}(t)$, and that the possible poles of $\mu \mapsto U_{t}^{\mu}(t)$ are at $t_{j} q^{\mathbb{Z}}(j=1,8)$. Without loss of generality we assume the generic conditions on the parameters $t \in \mathbb{C}^{8}\left(\prod_{j=1}^{8} t_{j}=1\right)$ such that $U_{t}^{\mu}(t)$ admits the integral representation as above, and such that $t_{1} \notin t_{8} q^{\mathbb{Z}}$. The latter condition ensures that the possible poles $t_{1} q^{\mathbb{Z}}, t_{8} q^{\mathbb{Z}}$ of $\mu \mapsto U_{t}^{\mu}(t)$ are at most simple. But the residue of $U_{t}^{\mu}(t)$ at $\mu=t_{j}(j=1,8)$ is zero, since it is an integral over a deformation $\mathcal{C}^{\prime}$ of $\mathbb{T}$ whose integrand is analytic within the integration contour $\mathcal{C}^{\prime}$ and vanishes at the origin. Hence $\mathbb{C}^{\times} \ni \mu \mapsto U_{t}^{\mu}(t)$ is bounded and analytic, hence constant by Liouville's Theorem. 
In view of Lemma 5.2, we omit the $\mu$-dependence in the notation for $U_{t}^{\mu}(t)$. Since $I_{t}(-t ; z)=I_{t}(t ;-z)$ and $J_{t}^{\mu}(-t ; z)=J_{t}^{-\mu}(t ;-z)$, we may and will view $S_{t}$ and $U_{t}$ as meromorphic function on $\mathcal{H}_{1}$.

By choosing a special value of $\mu$, we are able to derive another, "unfolded" integral representation of $U_{t}(t)$ as follows. Let $\mathbb{H}_{+}$be the upper half plane in $\mathbb{C}$. Choose $\tau \in \mathbb{H}_{+}$such that $q=e(\tau)$, where $e(x)$ is a shorthand notation for $\exp (2 \pi i x)$. Recall the surjective map $\psi_{0}: \mathcal{G}_{0} \rightarrow \mathcal{H}_{1}$ from Sect. 2 .

Corollary 5.3. For generic parameters $u \in \mathcal{G}_{0}$ we have

$$
\begin{aligned}
& U_{t}\left(\psi_{0}(2 \pi i u)\right) \\
& =\frac{2}{\tau(q, q ; q)_{\infty}} \frac{\left(e\left(\left(u_{8}-u_{1}\right) / \tau\right)-1\right)}{e\left(u_{1}\right) \theta\left(e\left(u_{8}-u_{1}\right) ; q\right)} \\
& \quad \times \int_{\mathcal{L}}\left\{\left(1-\frac{e(2 x)}{q}\right) \prod_{j=2}^{7} \frac{\left(e\left(x-u_{j}\right) ; q\right)_{\infty}}{\left(e\left(x+u_{j}\right) ; q\right)_{\infty}} \frac{\left(q e\left(x-u_{1}\right), q e\left(x-u_{8}\right) ; q\right)_{\infty}}{\left(q^{-1} e\left(x+u_{1}\right), q^{-1} e\left(x+u_{8}\right) ; q\right)_{\infty}}\right. \\
& \left.\quad \times \frac{e(x)}{\left(1-e\left(\left(u_{8}-x\right) / \tau\right)\right)\left(1-e\left(\left(x-u_{1}\right) / \tau\right)\right)}\right\} d x,
\end{aligned}
$$

where the integration contour $\mathcal{L}$ is some translate $\xi+\mathbb{R}(\xi \in i \mathbb{R})$ of the real line with a finite number of indentations, such that $\mathcal{C}$ separates the pole sequences $-u_{1}+\mathbb{Z}+\mathbb{Z} \leq 1 \tau$, $-u_{8}+\mathbb{Z}+\mathbb{Z}_{\leq 1} \tau$ and $-u_{j}+\mathbb{Z}+\mathbb{Z}_{\leq 0} \tau(j=2, \ldots, 7)$ of the integrand from the pole sequences $u_{1}+\mathbb{Z}_{\geq 0} \tau$ and $u_{8}+\mathbb{Z}_{\geq 0} \tau$.

Remark 5.4. Note that always $\xi \neq 0$ in Corollary 5.3. Due to the balancing condition $\sum_{j=1}^{8} u_{j}=0$, there are no parameter choices for which $\mathcal{L}=\mathbb{R}$ can be taken as an integration contour. This is a reflection of the fact that there are no parameters $t \in \mathcal{H}_{1}$ such that the unit circle $\mathbb{T}$ can be chosen as an integration cycle in the original integral representation $U_{t}(t)=\int_{\mathcal{C}^{\prime}} J_{t}^{\mu}(t ; z) \frac{d z}{2 \pi i z}$ of $U_{t}(t)$.

Proof. In the integral expression

$$
U_{t}\left(\psi_{c}(2 \pi i u)\right)=\int_{\mathcal{C}^{\prime}} J_{t}^{\mu}\left(\psi_{0}(2 \pi i u) ; z\right) \frac{d z}{2 \pi i z},
$$

we change the integration variable to $z=e(x)$, take $\mu=e\left(\eta\left(u_{1}, u_{8}\right)\right)$, and we use Lemma 5.1 to rewrite the quotient of theta-functions in the integrand as a bilateral sum. Changing the integration over the indented line segment with the bilateral sum using Fubini's Theorem, we can rewrite the resulting expression as a single integral over a noncompact integration cycle $\mathcal{L}$. This leads directly to the desired result.

In the following lemma we show that $U_{t}(t)$ can be expressed as a sum of two nonterminating very-well-poised ${ }_{10} \phi_{9}$ series.

Lemma 5.5. As meromorphic functions in $t \in \mathcal{H}_{1}$, we have

$$
\begin{aligned}
U_{t}(t)= & \frac{2}{\left(q, t_{1}^{2}, t_{1} t_{8} / q, t_{8} / t_{1} ; q\right)_{\infty}} \prod_{j=2}^{7} \frac{\left(t_{1} / t_{j} ; q\right)_{\infty}}{\left(t_{1} t_{j} ; q\right)_{\infty}} \\
& \times{ }_{10} W_{9}\left(\frac{t_{1}^{2}}{q} ; t_{1} t_{2}, t_{1} t_{3}, \ldots, t_{1} t_{7}, \frac{t_{1} t_{8}}{q} ; q, q\right)+\left(t_{1} \leftrightarrow t_{8}\right) .
\end{aligned}
$$


Proof. For generic $t \in \mathcal{H}_{1}$ we shrink the contour $\mathcal{C}^{\prime}$ in the integral representation of $U_{t}^{\mu}(t)=\int_{\mathcal{C}^{\prime}} J_{t}^{\mu}(t ; z) \frac{d z}{2 \pi i z}$ to the origin while picking up the residues at the pole sequences $t_{1} q^{\mathbb{Z}_{\geq 0}}$ and $t_{8} q^{\mathbb{Z} \geq 0}$ of the integrand $J_{t}^{\mu}(t ; z)$. The resulting sum of residues can be directly rewritten as a sum of two very-well-poised ${ }_{10} \phi_{9}$ series, leading to the desired identity (cf. the general residue techniques in $[5, \S 4.10]$ ).

Remark 5.6. Lemma 5.5 yields that $U_{t}(t)$ is, up to an explicit rescaling factor, an integral form of the particular sum $\Phi$ of two very-well-poised ${ }_{10} \phi_{9}$ series as e.g. studied in [7] and [15] (see [7, (1.8)], [15, (9c)]). Note furthermore that the explicit $\mu$-dependent quotient of theta-functions in the integrand of $U_{t}^{\mu}(t)$ has the effect that it balances the very-well-poised ${ }_{10} \phi_{9}$ series when picking up the residues of $J_{t}^{\mu}(t ; z)$ at the two pole sequences $t_{1} q^{\mathbb{Z}_{\geq 0}}$ and $t_{8} q^{\mathbb{Z}_{\geq 0}}$.

In the following proposition we show that $S_{t}$ (respectively $U_{t}$ ) is the degeneration of $S_{e}$ along the root vector $\alpha_{18}^{+}$(respectively $\left.\gamma_{18}\right)$.

Proposition 5.7. Let $t=\left(t_{1}, \ldots, t_{8}\right) \in \mathbb{C}^{8}$ be generic parameters satisfying the balancing condition $\prod_{j=1}^{8} t_{j}=1$. Then

$$
\begin{aligned}
S_{t}(t) & =\lim _{p \rightarrow 0} S_{e}\left(p q t_{1}, t_{2}, \ldots, t_{7}, p q t_{8}\right), \\
U_{t}(t) & =\lim _{p \rightarrow 0} \theta\left(t_{1} t_{8} / p q ; q\right) S_{e}\left((p q)^{-\frac{1}{2}} t_{1},(p q)^{\frac{1}{2}} t_{2}, \ldots,(p q)^{\frac{1}{2}} t_{7},(p q)^{-\frac{1}{2}} t_{8}\right) .
\end{aligned}
$$

Proof. For the degeneration to $S_{t}(t)$ we use that

$$
I_{e}\left(p q t_{1}, t_{2}, \ldots, t_{7}, p q t_{8} ; z\right)=\frac{\prod_{j=2}^{7} \Gamma_{e}\left(t_{j} z^{ \pm 1} ; p, q\right)}{\Gamma_{e}\left(z^{ \pm 2}, t_{1}^{-1} z^{ \pm 1}, t_{8}^{-1} z^{ \pm 1} ; p, q\right)}
$$

in view of the reflection equation for $\Gamma_{e}$, which (pointwise) tends to $I_{t}(t ; z)$ as $p \rightarrow 0$ in view of (5.1). A standard application of Lebesgue's dominated convergence theorem leads to the limit of the associated integrals.

The degeneration to $U_{t}(t)$ is more involved, since one needs to use a nontrivial symmetry argument to cancel some unwanted sequences of poles of $I_{e}(t ; z)$. To ease the notations we set

$$
t_{p}=\left((p q)^{-\frac{1}{2}} t_{1},(p q)^{\frac{1}{2}} t_{2}, \ldots,(p q)^{\frac{1}{2}} t_{7},(p q)^{-\frac{1}{2}} t_{8}\right)
$$

and we denote

$$
Q(z)=\frac{\theta\left((p q)^{-\frac{1}{2}} t_{1} t_{8} / \mu z,(p q)^{-\frac{1}{2}} t_{1} z,(p q)^{-\frac{1}{2}} t_{8} z,(p q)^{\frac{1}{2}} z / \mu ; q\right)}{\theta\left(z^{2} ; q\right)} .
$$

By (3.6), we have the identity

$$
Q(z)+Q\left(z^{-1}\right)=\theta\left(t_{1} t_{8} / p q, t_{1} / \mu, t_{8} \mu ; q\right) .
$$

Since the integrand $I_{e}\left(\tau_{p} ; z\right)$ is invariant under $z \mapsto z^{-1}$, we can consequently write

$$
\theta\left(t_{1} t_{8} / p q ; q\right) S_{e}\left(t_{p} ; z\right)=\frac{2}{\theta\left(t_{1} / \mu, t_{8} / \mu ; q\right)} \int_{\mathcal{C}} Q(z) I_{e}\left(t_{p} ; z\right) \frac{d z}{2 \pi i z},
$$


with $\mathcal{C}$ a deformation of the positively oriented unit circle $\mathbb{T}$ separating the downward pole sequences of the integrand from the upward pole sequences. Taking $(p q)^{\frac{1}{2}} z$ as a new integration variable and using the functional equation and reflection equation of $\Gamma_{e}$, we obtain the integral representation

$$
\begin{aligned}
\theta\left(t_{1} t_{8} / p q ; q\right) S_{e}\left(t_{p} ; z\right)= & 2 \int_{\mathcal{C}} \frac{\theta\left(t_{1} t_{8} / \mu z, z / \mu ; q\right)}{\theta\left(t_{1} / \mu, t_{8} / \mu ; q\right)} \theta\left(z^{2} / q ; p\right) \prod_{j=2}^{7} \frac{\Gamma_{e}\left(t_{j} z ; p, q\right)}{\Gamma_{e}\left(z / t_{j} ; p, q\right)} \\
& \times \prod_{j=1,8} \Gamma_{e}\left(t_{j} z / q, t_{j} / z ; p, q\right) \frac{d z}{2 \pi i z},
\end{aligned}
$$

where $\mathcal{C}$ is a deformation of the positively oriented unit circle $\mathbb{T}$ which includes the pole sequences $t_{1} p^{\mathbb{Z}_{\geq 0}} q^{\mathbb{Z}_{\geq 0}}, t_{8} p^{\mathbb{Z}_{\geq 0}} q^{\mathbb{Z}_{\geq 0}}$ and $t_{j} p^{\mathbb{Z}_{\geq 1}} q^{\mathbb{Z}_{\geq 1}}(j=2, \ldots, 7)$, and which excludes the pole sequences $t_{1}^{-1} p^{\mathbb{Z}_{\leq 0}} q^{\mathbb{Z}_{\leq 1}}, t_{8}^{-1} p^{\mathbb{Z}_{\leq 0}} q^{\mathbb{Z}_{\leq 1}}$ and $t_{j}^{-1} p^{\mathbb{Z}_{\leq 0}} q^{\mathbb{Z}_{\leq 0}}(j=2, \ldots, 7)$. We can now take the limit $p \rightarrow 0$ in (5.6) with $p$-independent, fixed integration contour $\mathcal{C}$, leading to the desired limit relation

$$
\lim _{p \rightarrow 0} \theta\left(t_{1} t_{8} / p q ; q\right) S_{e}\left(t_{p}\right)=U_{t}^{\mu}(t)
$$

Remark 5.8. Observe that Lemma 5.5 and the proof of Proposition 5.7 entail independent proofs of Lemma 5.2.

By specializing the parameters $t \in \mathcal{H}_{1}$ in Proposition 5.7 further, we arrive at trigonometric integrals which can be evaluated by (3.2). The resulting trigonometric degenerations lead immediately to the trigonometric Nassrallah-Rahman integral evaluation formula [5, (6.4.1)] and Gasper's integral evaluation formula [5, (4.11.4)]:

Corollary 5.9. For generic $t=\left(t_{1}, \ldots, t_{6}\right) \in \mathbb{C}^{6}$ satisfying the balancing condition $\prod_{j=1}^{6} t_{j}=1$ we have

$$
\begin{gathered}
\int_{\mathcal{C}} \frac{\left(z^{ \pm 2}, t_{1}^{-1} z^{ \pm 1} ; q\right)_{\infty}}{\prod_{j=2}^{6}\left(t_{j} z^{ \pm 1} ; q\right)_{\infty}} \frac{d z}{2 \pi i z}=\frac{2 \prod_{j=2}^{6}\left(1 / t_{1} t_{j} ; q\right)_{\infty}}{(q ; q)_{\infty} \prod_{2 \leq j<k \leq 6}\left(t_{j} t_{k} ; q\right)_{\infty}}, \\
\int_{\mathcal{C}^{\prime}} \frac{\theta\left(t_{5} t_{6} / \mu z, z / \mu ; q\right)}{\theta\left(t_{5} / \mu, t_{6} / \mu ; q\right)}\left(1-\frac{z^{2}}{q}\right) \prod_{j=1}^{4} \frac{\left(z / t_{j} ; q\right)_{\infty}}{\left(t_{j} z ; q\right)_{\infty}} \prod_{k=5,6} \frac{1}{\left(t_{k} z / q, t_{k} / z ; q\right)_{\infty}} \frac{d z}{2 \pi i z}= \\
=\frac{\prod_{1 \leq j<k \leq 4}\left(1 / t_{j} t_{k} ; q\right)_{\infty}}{\left(q, t_{5} t_{6} / q ; q\right)_{\infty} \prod_{j=1}^{4} \prod_{k=5}^{6}\left(t_{j} t_{k} ; q\right)_{\infty}},
\end{gathered}
$$

where $\mathcal{C}$ is the deformation of $\mathbb{T}$ separating the pole sequences $t_{j} q^{\mathbb{Z}_{\geq 0}}(j=2, \ldots, 6)$ of the integrand from their reciprocals, and where $\mathcal{C}^{\prime}$ is the deformation of $\mathbb{T}$ separating the pole sequences $t_{5} q^{\mathbb{Z}_{\geq 0}}, t_{6} q^{\mathbb{Z}_{\geq 0}}$ of the integrand from the pole sequences $t_{j}^{-1} q^{\mathbb{Z}_{\leq 0}}$ ( $j=1, \ldots, 4), t_{5}^{-1} q^{\mathbb{Z}_{\leq 1}}$ and $t_{6}^{-1} q^{\mathbb{Z}_{\leq 1}}$. 
Proof. For the first integral evaluation, take $t \in \mathcal{H}_{1}$ and $t_{7}=t_{8}^{-1}$ in the degeneration from $S_{e}$ to $S_{t}$, and use the elliptic Nassrallah-Rahman integral evaluation formula (3.2).

For the second integral evaluation, take $t \in \mathcal{H}_{1}$ with $t_{6}=t_{7}^{-1}$ in the degeneration from $S_{e}$ to $U_{t}^{\mu}$ and again use (3.2) to evaluate the elliptic integral. It leads to the second integral evaluation formula with generic parameters $\left(t_{1}, t_{2}, t_{3}, t_{4}, t_{5}, t_{8}\right) \in \mathbb{C}^{6}$ satisfying $t_{1} \cdots t_{5} t_{8}=1$.

The second integral in Corollary 5.9 can be unfolded using Lemma 5.1 as in Corollary 5.3. We obtain for generic parameters $u \in \mathbb{C}^{6}$ satisfying $\sum_{j=1}^{6} u_{j}=0$,

$$
\begin{aligned}
\int_{\mathcal{L}} & \left\{\left(1-\frac{e(2 x)}{q}\right) \prod_{j=1}^{4} \frac{\left(e\left(x-u_{j}\right) ; q\right)_{\infty}}{\left(e\left(x+u_{j}\right) ; q\right)_{\infty}} \frac{\left(q e\left(x-u_{5}\right), q e\left(x-u_{6}\right) ; q\right)_{\infty}}{\left(q^{-1} e\left(x+u_{5}\right), q^{-1} e\left(x+u_{6}\right) ; q\right)_{\infty}}\right. \\
& \left.\times \frac{e(x)}{\left(1-e\left(\left(u_{6}-x\right) / \tau\right)\right)\left(1-e\left(\left(x-u_{5}\right) / \tau\right)\right)}\right\} d x \\
= & \frac{\tau t_{5} \theta\left(t_{6} / t_{5} ; q\right)}{\left(e\left(\left(u_{6}-u_{5}\right) / \tau\right)-1\right)} \frac{(q ; q)_{\infty} \prod_{1 \leq j<k \leq 4}\left(1 / t_{j} t_{k} ; q\right)_{\infty}}{\left(t_{5} t_{6} / q\right)_{\infty} \prod_{j=1}^{4} \prod_{k=5}^{6}\left(t_{j} t_{k} ; q\right)_{\infty}},
\end{aligned}
$$

where $\tau \in \mathbb{H}_{+}$such that $q=e(\tau)$, where $t_{j}=e\left(u_{j}\right)(j=1, \ldots, 6)$ and where the integration contour $\mathcal{L}$ is some translate $\xi+\mathbb{R}(\xi \in i \mathbb{R})$ of the real line with a finite number of indentations, such that $\mathcal{C}$ separates the pole sequences $-u_{5}+\mathbb{Z}+\mathbb{Z}_{\leq 1} \tau,-u_{6}+\mathbb{Z}+\mathbb{Z}_{\leq 1} \tau$ and $-u_{j}+\mathbb{Z}+\mathbb{Z}_{\leq 0} \tau(j=1, \ldots, 4)$ of the integrand from the pole sequences $u_{5}+\mathbb{Z}_{\geq 0} \tau$ and $u_{6}+\mathbb{Z}_{\geq 0} \tau$. This is Agarwal's identity [5, (4.7.5)].

Furthermore, using Lemma 5.5 the second integral in Corollary 5.9 can be written as a sum of two very-well-poised ${ }_{8} \phi_{7}$-series. We obtain for generic $t \in \mathbb{C}^{6}$ satisfying $\prod_{j=1}^{6} t_{j}=1$,

$$
\begin{gathered}
\frac{1}{\left(q, t_{5}^{2}, t_{5} t_{6} / q, t_{6} / t_{5} ; q\right)_{\infty}} \prod_{j=1}^{4} \frac{\left(t_{5} / t_{j} ; q\right)_{\infty}}{\left(t_{5} t_{j} ; q\right)_{\infty}}{ }_{8} W_{7}\left(\frac{t_{5}^{2}}{q} ; t_{5} t_{1}, t_{5} t_{2}, t_{5} t_{3}, t_{5} t_{4}, \frac{t_{5} t_{6}}{q} ; q, q\right)+\left(t_{5} \leftrightarrow t_{6}\right) \\
=\frac{\prod_{1 \leq j<k \leq 4}\left(1 / t_{j} t_{k} ; q\right)_{\infty}}{\left(q, t_{5} t_{6} / q ; q\right)_{\infty} \prod_{j=1}^{4} \prod_{k=5}^{6}\left(t_{j} t_{k} ; q\right)_{\infty}},
\end{gathered}
$$

which is Bailey's summation formula [5, (2.11.7)] of the sum of two very-well-poised ${ }_{8} \phi_{7}$ series.

We can now compute the (nontrivial) $W\left(E_{6}\right)$-symmetries of the trigonometric hypergeometric integrals $S_{t}$ and $U_{t}$ by taking limits of the corresponding symmetries on the elliptic level using Proposition 5.7. We prefer to give a derivation based on the trigonometric evaluation formulas (see Corollary 5.9), in analogy to our approach in the elliptic and hyperbolic cases.

Proposition 5.10. The trigonometric integrals $S_{t}(t)$ and $U_{t}(t)\left(t \in \mathcal{H}_{1}\right)$ are invariant under permutations of $\left(t_{1}, t_{8}\right)$ and of $\left(t_{2}, \ldots, t_{7}\right)$. Furthermore,

$$
\begin{aligned}
S_{t}(t) & =S_{t}(w t) \frac{\left(1 / t_{1} t_{2}, 1 / t_{1} t_{3}, 1 / t_{1} t_{4}, 1 / t_{8} t_{5}, 1 / t_{8} t_{6}, 1 / t_{8} t_{7} ; q\right)_{\infty}}{\left(t_{2} t_{3}, t_{2} t_{4}, t_{3} t_{4}, t_{5} t_{6}, t_{5} t_{7}, t_{6} t_{7} ; q\right)_{\infty}}, \\
U_{t}(t) & =U_{t}(w t) \frac{\left(1 / t_{2} t_{3}, 1 / t_{2} t_{4}, 1 / t_{3} t_{4}, 1 / t_{5} t_{6}, 1 / t_{5} t_{7}, 1 / t_{6} t_{7} ; q\right)_{\infty}}{\left(t_{1} t_{2}, t_{1} t_{3}, t_{1} t_{4}, t_{5} t_{8}, t_{6} t_{8}, t_{7} t_{8} ; q\right)_{\infty}}
\end{aligned}
$$


as meromorphic functions in $t \in \mathcal{H}_{1}$.

Proof. In order to derive the $w$-symmetry of $S_{t}(t)$ we consider the double integral

$$
\int_{\mathbb{T}^{2}} \frac{\left(z^{ \pm 2}, x^{ \pm 2}, t_{1}^{-1} z^{ \pm 1}, s t_{8}^{-1} x^{ \pm 1} ; q\right)_{\infty}}{\left(t_{2} z^{ \pm 1}, t_{3} z^{ \pm 1}, t_{4} z^{ \pm 1}, s z^{ \pm 1} x^{ \pm 1}, s^{-1} t_{5} x^{ \pm 1}, s^{-1} t_{6} x^{ \pm 1}, s^{-1} t_{7} x^{ \pm 1} ; q\right)_{\infty}} \frac{d z}{2 \pi i z} \frac{d x}{2 \pi i x}
$$

for parameters $\left(t_{1}, \ldots, t_{8}\right) \in \mathbb{C}^{8}$ satisfying $\prod_{j=1}^{8} t_{j}=1$, where $s^{2} t_{1} t_{2} t_{3} t_{4}=1=$ $s^{-2} t_{5} t_{6} t_{7} t_{8}$ and where we assume the additional parameter restraints

$$
\left|t_{2}\right|,\left|t_{3}\right|,\left|t_{4}\right|,|s|,\left|t_{5} / s\right|,\left|t_{6} / s\right|,\left|t_{7} / s\right|<1
$$

to ensure that the integration contour $\mathbb{T}$ separates the downward sequences of poles from the upward sequences. The desired transformation then follows by either integrating the double integral first to $x$, or first to $z$, using in each case the trigonometric NassrallahRahman integral evaluation formula (see Corollary 5.9).

The proof of the $w$-symmetry of $U_{t}(t)$ follows the same line of arguments. For $\epsilon>0$ we denote $\epsilon \mathbb{T}$ for the positively oriented circle in the complex plane with radius $\epsilon$ and centered at the origin. The $w$-symmetry

$$
U_{t}^{\mu / s}(t)=U_{t}^{\mu}(w t) \frac{\left(1 / t_{2} t_{3}, 1 / t_{2} t_{4}, 1 / t_{3} t_{4}, 1 / t_{5} t_{6}, 1 / t_{5} t_{7}, 1 / t_{6} t_{7} ; q\right)_{\infty}}{\left(t_{1} t_{2}, t_{1} t_{3}, t_{1} t_{4}, t_{5} t_{8}, t_{6} t_{8}, t_{7} t_{8} ; q\right)_{\infty}}
$$

for $t \in \mathcal{H}_{1}$, where $s^{2} t_{1} t_{2} t_{3} t_{4}=1=s^{-2} t_{5} t_{6} t_{7} t_{8}$, by considering for $\left(t_{1}, \ldots, t_{8}\right) \in \mathbb{C}^{8}$ satisfying $\prod_{j=1}^{8} t_{j}=1$ the double integral

$$
\begin{aligned}
& \int_{(|q s| \mathbb{T})^{2}}\left\{\frac{\theta\left(s t_{1} t_{8} / \mu z, t_{8} z / \mu x, x / \mu ; q\right)}{\theta\left(s t_{8} / \mu, s t_{1} / \mu, t_{8} / s \mu ; q\right)}\left(1-\frac{z^{2}}{q}\right)\left(1-\frac{x^{2}}{q}\right)\right. \\
& \left.\quad \times \frac{\left(z / t_{2}, z / t_{3}, z / t_{4}, x z / s, s x / t_{5}, s x / t_{6}, s x / t_{7} ; q\right)_{\infty}}{\left(t_{1} z / q, t_{1} / z, t_{2} z, t_{3} z, t_{4} z, s x z / q, s z / x, s x / z, t_{5} x / s, t_{6} x / s, t_{7} x / s, t_{8} x / q s, t_{8} / s x ; q\right)_{\infty}}\right\} \frac{d z}{2 \pi i z} \frac{d x}{2 \pi i x}
\end{aligned}
$$

with $s^{2} t_{1} t_{2} t_{3} t_{4}=1=s^{-2} t_{5} t_{6} t_{7} t_{8}$, where we assume the additional parameter restraints

$0<|s| \ll\left|q^{\frac{1}{2}}\right|, \quad\left|t_{1}\right|,\left|t_{2}^{-1}\right|,\left|t_{3}^{-1}\right|,\left|t_{4}^{-1}\right|<|q s|, \quad\left|t_{5}\right|,\left|t_{6}\right|,\left|t_{7}\right|<\left|q^{-1}\right|, \quad\left|t_{8}\right|<\left|q s^{2}\right|$

to ensure a proper separation by the integration contours of the upward sequences of poles from the downward sequences. Using the second trigonometric integral evaluation formula of Corollary 5.9 then yields the desired result for the restricted parameter domain. Analytic continuation completes the proof.

Remark 5.11. Rewriting $U_{t}(t)$ as a sum of two very-well-poised ${ }_{10} \phi_{9}$ series (see Lemma 5.5 and Remark 5.6), the $w$-symmetry of $U_{t}(t)$ becomes Bailey's four-term transformation formula [5, (2.12.9)], see also [7]. The identification of the symmetry group of $U_{t}$ with the Weyl group of type $E_{6}$ has been derived by different methods in [15].

Finally we relate the two trigonometric integrals $S_{t}$ and $U_{t}$. We can obtain the following proposition as a degeneration of a particular $W\left(E_{7}\right)$-symmetry of $S_{e}$, but we prefer here to give a direct proof using double integrals. 
Proposition 5.12. As a meromorphic function in $t \in \mathcal{H}_{1}$, we have $S_{t}(t) \frac{\prod_{2 \leq j<k \leq 5}\left(t_{j} t_{k} ; q\right)_{\infty}\left(t_{6} t_{7} ; q\right)_{\infty}}{\left(1 / q t_{1} t_{8}, 1 / t_{1} t_{6}, 1 / t_{1} t_{7}, 1 / t_{8} t_{6}, 1 / t_{8} t_{7} ; q\right)_{\infty}}=U_{t}\left(t_{6} / s, s t_{2}, s t_{3}, s t_{4}, s t_{5}, t_{1} / s, t_{8} / s, t_{7} / s\right)$, where $t_{2} t_{3} t_{4} t_{5} s^{2}=1=t_{1} t_{6} t_{7} t_{8} / s^{2}$

Proof. For $\left(t_{1}, \ldots, t_{8}\right) \in \mathbb{C}^{8}$ satisfying $\prod_{j=1}^{8} t_{j}=1$ we consider the double integral

$$
\begin{aligned}
& \int_{z \in \eta \mathbb{T}} \int_{x \in \mathbb{T}} \frac{\theta\left(\mu z, t_{6} t_{7} \mu / s^{2} z\right)}{\theta\left(t_{6} \mu / s, t_{7} \mu / s\right)}\left(1-\frac{z^{2}}{q}\right) \prod_{j=2}^{5} \frac{1}{\left(t_{j} x^{ \pm 1} ; q\right)_{\infty}} \\
& \times \frac{\left(x^{ \pm 2}, z x^{ \pm 1} / s, s z / t_{1}, s z / t_{8} ; q\right)_{\infty}}{\left(s z x^{ \pm 1}, t_{7} z / s q, t_{7} / s z, t_{6} z / s q, t_{6} / s z, t_{1} z / s, t_{8} z / s ; q\right)_{\infty}} \frac{d x}{2 \pi i x} \frac{d z}{2 \pi i z}
\end{aligned}
$$

with $s^{2} t_{2} t_{3} t_{4} t_{5}=1=s^{-2} t_{1} t_{6} t_{7} t_{8}$ and with $0<\eta<\min \left(\left|s^{-1}\right|,\left|q^{\frac{1}{2}}\right|\right)$, where we assume the additional parameter restraints

$$
\left|t_{2}\right|,\left|t_{3}\right|,\left|t_{4}\right|,\left|t_{5}\right|<1, \quad\left|t_{6}\right|,\left|t_{7}\right|<\eta|s|, \quad\left|t_{1}\right|,\left|t_{8}\right|<\eta^{-1}|s|
$$

to ensure a proper separation by the integration contours of the upward sequences of poles from the downward sequences. Using Corollary 5.9, we can first integrate over $x$ using the trigonometric Nassrallah-Rahman integral evaluation formula, or first integrate over $z$ using the second integral evaluation formula of Corollary 5.9. The resulting identity gives the desired result for restricted parameter values. Analytic continuation completes the proof.

Remark 5.13. (i) Combining Proposition 5.12 with Lemma 5.5 we obtain an expression of $S_{t}(t)$ as a sum of two very-well-poised ${ }_{10} \phi_{9}$ series, which is originally due to Rahman [5, (6.4.8)].

(ii) For e.g. $t_{1} t_{6}=q^{m}\left(m \in \mathbb{Z}_{\geq 0}\right)$, it follows from (i) (see also [20] and [5, (6.4.10)]) that the $S_{t}(t ; p, q)$ essentially coincides with the biorthogonal rational function of Rahman [20], which is explicitly given as a terminating very-well-poised ${ }_{10} \phi_{9}$ series.

5.3. Contiguous relations. The fundamental equation on this level equals

$$
\frac{1}{y}\left(1-v x^{ \pm 1}\right)\left(1-y z^{ \pm 1}\right)+\frac{1}{z}\left(1-v y^{ \pm 1}\right)\left(1-z x^{ \pm 1}\right)+\frac{1}{x}\left(1-v z^{ \pm 1}\right)\left(1-x y^{ \pm 1}\right)=0,
$$

where $\left(1-a x^{ \pm 1}\right)=(1-a x)\left(1-a x^{-1}\right)$. The fundamental relation (5.8) is the $p=0$ reduction of (3.6). In this section $\tau_{i j}=\tau_{i j}^{-\log (q)}$ acts as in the elliptic case by multiplying $t_{i}$ by $q$ and dividing $t_{j}$ by $q$. Formula (5.8) leads as in the elliptic case to the difference equation

$$
\frac{\left(1-t_{5} t_{6}^{ \pm 1} / q\right)}{\left(1-t_{4} t_{6}^{ \pm 1}\right)} S_{t}\left(\tau_{45} t\right)+\frac{\left(1-t_{5} t_{4}^{ \pm 1} / q\right)}{\left(1-t_{6} t_{4}^{ \pm 1}\right)} S_{t}\left(\tau_{65} t\right)=S_{t}(t), \quad t \in \mathcal{H}_{1} .
$$


To obtain a second difference equation between trigonometric hypergeometric functions where two times the same parameter is multiplied by $q$, we can mimic the approach in the elliptic case with the role of the longest Weyl group element taken over by the element $u=w_{35} s_{46} w \in W\left(E_{6}\right)$. Alternatively, one can rewrite the difference equation (3.8) for $S_{e}$ in the form

$$
\begin{aligned}
& \frac{\theta\left(t_{3} / q t_{4}, 1 / t_{1} t_{5}, 1 / t_{8} t_{5}, t_{2} t_{5} / q, t_{5} t_{6} / q, t_{5} t_{7} / q ; p\right)}{\theta\left(t_{3} / t_{5} ; p\right)} S_{e}\left(\tau_{45} \widetilde{t}\right)+\left(t_{3} \leftrightarrow t_{5}\right) \\
& \quad=\theta\left(1 / q t_{1} t_{4}, 1 / q t_{8} t_{4}, t_{2} t_{4}, t_{4} t_{6}, t_{4} t_{7} ; p\right) S_{e}(\widetilde{t})
\end{aligned}
$$

where $t \in \mathcal{H}_{1}$ and $\widetilde{t}=\left(p q t_{1}, t_{2}, \ldots, t_{7}, p q t_{8}\right)$, and degenerate it using Proposition 5.7. We arrive at

$$
\begin{aligned}
& \frac{\left(1-t_{3} / q t_{4}\right)}{\left(1-t_{3} / t_{5}\right)} \prod_{j=1,8} \frac{\left(1-1 / t_{5} t_{j}\right)}{\left(1-1 / q t_{4} t_{j}\right)} \prod_{j=2,6,7} \frac{\left(1-t_{5} t_{j} / q\right)}{\left(1-t_{4} t_{j}\right)} S_{t}\left(\tau_{45} t\right) \\
& +\left(t_{3} \leftrightarrow t_{5}\right)=S_{t}(t), \quad t \in \mathcal{H}_{1} .
\end{aligned}
$$

Together these equations imply the following result.

Proposition 5.14. We have

$$
A(t) S_{t}\left(\tau_{45} t\right)+\left(t_{4} \leftrightarrow t_{5}\right)=B(t) S_{t}(t)
$$

as meromorphic functions in $t \in \mathcal{H}_{1}$, where

$$
\begin{aligned}
A(t)= & -\frac{\left(1-\frac{1}{t_{1} t_{5}}\right)\left(1-\frac{1}{t_{8} t_{5}}\right) \prod_{j=2,3,6,7}\left(1-\frac{t_{5} t_{j}}{q}\right)}{t_{4}\left(1-\frac{t_{4} t_{5}}{q}\right)\left(1-\frac{t_{5}}{q t_{4}}\right)\left(1-\frac{t_{4}}{t_{5}}\right)}, \\
B(t)=- & \frac{\left(1-\frac{1}{q t_{1} t_{6}}\right)\left(1-\frac{1}{q t_{8} t_{6}}\right)\left(1-t_{3} t_{6}\right)\left(1-t_{7} t_{6}\right)\left(1-t_{2} t_{6}\right)}{t_{6}\left(1-\frac{t_{4}}{q t_{6}}\right)\left(1-\frac{t_{5}}{q t_{6}}\right)} \\
& +\frac{\left(1-\frac{t_{6}}{t_{4}}\right)\left(1-t_{6} t_{4}\right) \prod_{j=1,8}\left(1-\frac{1}{t_{j} t_{5}}\right) \prod_{j=2,3,7}\left(1-\frac{t_{j} t_{5}}{q}\right)}{t_{6}\left(1-\frac{t_{5}}{q t_{6}}\right)\left(1-\frac{t_{4} t_{5}}{q}\right)\left(1-\frac{t_{5}}{q t_{4}}\right)\left(1-\frac{t_{4}}{t_{5}}\right)} \\
& +\frac{\left(1-\frac{t_{6}}{t_{5}}\right)\left(1-t_{6} t_{5}\right) \prod_{j=1,8}\left(1-\frac{1}{t_{j} t_{4}}\right) \prod_{j=2,3,7}\left(1-\frac{t_{j} t_{4}}{q}\right)}{t_{6}\left(1-\frac{t_{4}}{q t_{6}}\right)\left(1-\frac{t_{4} t_{5}}{q}\right)\left(1-\frac{t_{4}}{q t_{5}}\right)\left(1-\frac{t_{5}}{t_{4}}\right)} .
\end{aligned}
$$

Despite the apparent asymmetric expression $B$ still satisfies $B\left(s_{67} t\right)=B(t)$.

The contiguous relation for the elliptic hypergeometric function $S_{e}$ with step-size $p$ can also be degenerated to the trigonometric level. A direct derivation is as follows. By (3.6) we have

$$
\begin{aligned}
& \frac{\theta\left(t_{8}^{-1} t_{7}^{ \pm 1} ; q\right)}{\theta\left(t_{6} t_{7}^{ \pm 1} ; q\right)} I_{t}\left(t_{1}, t_{2}, \ldots, t_{5}, q t_{8}, t_{7}, t_{6} / q ; z\right) \\
& \quad+\frac{\theta\left(t_{8}^{-1} t_{6}^{ \pm 1} ; q\right)}{\theta\left(t_{7} t_{6}^{ \pm 1} ; q\right)} I_{t}\left(t_{1}, t_{2}, \ldots, t_{6}, q t_{8}, t_{7} / q ; z\right)=I_{t}(t ; z) .
\end{aligned}
$$


Integrating this equation we obtain

$$
\frac{\theta\left(t_{8}^{-1} t_{7}^{ \pm 1} ; q\right)}{\theta\left(t_{6} t_{7}^{ \pm 1} ; q\right)} S_{t}\left(t_{1}, t_{2}, \ldots, t_{5}, q t_{8}, t_{7}, t_{6} / q\right)+\left(t_{6} \leftrightarrow t_{7}\right)=S_{t}(t)
$$

as meromorphic functions in $t \in \mathcal{H}_{1}$, a three term transformation for $S_{t}$. The three term transformation $[7,(6.5)]$ is equivalent to the sum of two equations of this type (in which the parameters are chosen such that two terms coincide and two other terms cancel each other).

Remark 5.15. In [15] it is shown that there are essentially five different types of three term transformations for $\Phi$ (see Remark 5.6), or equivalently of the integrals $U_{t}$ and $S_{t}$. The different types arise from a careful analysis of the three term transformations in terms of the $W\left(E_{7}\right)$-action on $\mathcal{H}_{1}$. It is likely that all five different types of three term transformations for $\Phi$ can be re-obtained by degenerating contiguous relations for $S_{e}$ with step-size $p$ (similarly as the derivation of (5.12)): concretely, the five prototypes are in one-to-one correspondence to the orbits of

$$
\left\{(\alpha, \beta, \gamma) \in \mathcal{O}^{3} \mid \alpha, \beta, \gamma \text { are pair-wise different }\right\}
$$

under the diagonal action of $W\left(E_{7}\right)$, where $\mathcal{O}$ is the $W\left(E_{7}\right)$-orbit (5.3).

5.4. Degenerations with $D_{5}$ symmetries. In this section we consider degenerations of $S_{t}$ and $U_{t}$ with symmetries with respect to the Weyl group of type $D_{5}$. Compared to the analysis on the hyperbolic level, we introduce a trigonometric analog of the Euler and Barnes' type integrals, as well as a third, new type of integral arising as degeneration of $U_{t}$. We first introduce the degenerate integrals explicitly.

For generic $t=\left(t_{1}, \ldots, t_{6}\right) \in\left(\mathbb{C}^{\times}\right)^{6}$ we define the trigonometric Euler integral as

$$
E_{t}(t)=\int_{\mathcal{C}} \frac{\left(z^{ \pm 2}, t_{1}^{-1} z^{ \pm 1} ; q\right)_{\infty}}{\prod_{j=2}^{6}\left(t_{j} z^{ \pm 1} ; q\right)_{\infty}} \frac{d z}{2 \pi i z}
$$

where $\mathcal{C}$ is a deformation of the positively oriented unit circle $\mathbb{T}$ separating the decreasing pole sequences $t_{j} q^{\mathbb{Z}_{\geq 0}}(j=2, \ldots, 6)$ of the integrand from their reciprocals. We have $E_{t}(-t)=E_{t}(t)$, and $E_{t}$ has a unique meromorphic extension to $\left(\mathbb{C}^{\times}\right)^{6}$. The resulting meromorphic function on $\left(\mathbb{C}^{\times}\right)^{6} / C_{2}$ is denoted also by $E_{t}$.

For generic $\mu \in \mathbb{C}^{\times}$and generic $t=\left(t_{1}, \ldots, t_{8}\right) \in \mathbb{C}^{8}$ satisfying the balancing condition $\prod_{j=1}^{8} t_{j}=1$ we define the trigonometric Barnes integral as

$$
B_{t}(t)=2 \int_{\mathcal{C}} \frac{\theta\left(t_{2} t_{7} / \mu z, z / \mu ; q\right)}{\theta\left(t_{2} / \mu, t_{7} / \mu ; q\right)} \frac{\left(z / t_{1}, z / t_{8} ; q\right)_{\infty}}{\prod_{j=3}^{6}\left(t_{j} z ; q\right)_{\infty}\left(t_{2} / z, t_{7} / z ; q\right)_{\infty}} \frac{d z}{2 \pi i z},
$$

where $\mathcal{C}$ is a deformation of $\mathbb{T}$ separating the decreasing pole sequences $t_{2} q^{\mathbb{Z}_{\geq 0}}$ and $t_{7} q^{\mathbb{Z}} \geq 0$ of the integrand from the increasing pole sequences $t_{j}^{-1} q^{\mathbb{Z}_{\leq 0}}(j=3, \ldots, 6)$. Analogously to the analysis of the integral $U_{t}(t)$, we have that the trigonometric Barnes integral $B_{t}(t)$ uniquely extends to a meromorphic function in $\left\{(\mu, t) \in \mathbb{C}^{\times} \times \mathbb{C}^{8} \mid \prod_{j=1}^{8} t_{j}=1\right\}$ 
which is independent of $\mu$ (cf. Lemma 5.2). Furthermore, by a change of integration variable we have $B_{t}(-t)=B_{t}(t)$, hence $B_{t}$ may (and will) be interpreted as meromorphic function on $\mathcal{H}_{1}$.

Finally, for generic $t=\left(t_{1}, \ldots, t_{6}\right) \in\left(\mathbb{C}^{\times}\right)^{6}$ we consider

$$
V_{t}(t)=2 \int_{\mathcal{C}} \frac{\theta\left(q t_{2} t_{3} t_{4} t_{5} t_{6} z ; q\right)}{\theta\left(q t_{1} t_{2} t_{3} t_{4} t_{5} t_{6} ; q\right)}\left(1-\frac{z^{2}}{q}\right) \prod_{j=2}^{6} \frac{\left(z / t_{j} ; q\right)_{\infty}}{\left(t_{j} z ; q\right)_{\infty}} \frac{1}{\left(t_{1} z / q, t_{1} / z ; q\right)_{\infty}} \frac{d z}{2 \pi i z},
$$

where $\mathcal{C}$ is a deformation of $\mathbb{T}$ separating the decreasing pole sequence $t_{1} q^{\mathbb{Z}_{\geq 0}}$ of the integrand from the remaining (increasing) pole sequences. As before, $V_{t}$ uniquely extends to a meromorphic function on $\left(\mathbb{C}^{\times}\right)^{6} / C_{2}$.

Similarly as for $U_{t}(t)$, the trigonometric Barnes integral $B_{t}(t)$ can be unfolded. Recall that $q=e(\tau)$ with $\tau \in \mathbb{H}_{+}$, where $e(x)$ is a shorthand notation for $\exp (2 \pi i x)$.

Lemma 5.16. For generic parameters $u \in \mathcal{G}_{0}$ we have

$$
\begin{aligned}
B_{t}\left(\psi_{0}(2 \pi i u)\right)= & \frac{2}{\tau(q, q ; q)_{\infty}} \frac{\left(e\left(\left(u_{7}-u_{2}\right) / \tau\right)-1\right)}{e\left(u_{2}\right) \theta\left(e\left(u_{7}-u_{2}\right) ; q\right)} \\
& \times \int_{\mathcal{L}}\left\{\frac{\left(e\left(x-u_{1}\right), q e\left(x-u_{2}\right), q e\left(x-u_{7}\right), e\left(x-u_{8}\right) ; q\right)_{\infty}}{\left(e\left(x+u_{3}\right), e\left(x+u_{4}\right), e\left(x+u_{5}\right), e\left(x+u_{6}\right) ; q\right)_{\infty}}\right. \\
& \left.\times \frac{e(x)}{\left(1-e\left(\left(u_{7}-x\right) / \tau\right)\right)\left(1-e\left(\left(x-u_{2}\right) / \tau\right)\right)}\right\} d x,
\end{aligned}
$$

where the integration contour $\mathcal{L}$ is some translate $\xi+\mathbb{R}(\xi \in i \mathbb{R})$ of the real line with a finite number of indentations, such that $\mathcal{C}$ separates the pole sequences $-u_{j}+\mathbb{Z}+\mathbb{Z}_{\leq 0} \tau$ $(j=3, \ldots, 6)$ of the integrand from the pole sequences $u_{2}+\mathbb{Z}_{\geq 0} \tau$ and $u_{7}+\mathbb{Z}_{\geq 0} \tau$.

Proof. The proof is similar to the proof of Corollary 5.3.

For $B_{t}(t)$ and $V_{t}(t)$ we have the following series expansions in balanced ${ }_{4} \phi_{3}$ 's (respectively in a very-well-poised $\left.{ }_{8} \phi_{7}\right)$.

Lemma 5.17. (a) We have

$$
B_{t}(t)=\frac{2\left(t_{2} / t_{1}, t_{2} / t_{8} ; q\right)_{\infty}}{\left(q, t_{7} / t_{2}, t_{2} t_{3}, t_{2} t_{4}, t_{2} t_{5}, t_{2} t_{6} ; q\right)_{\infty}}{ }_{4} \phi_{3}\left(\begin{array}{l}
t_{2} t_{3}, t_{2} t_{4}, t_{2} t_{5}, t_{2} t_{6} \\
q t_{2} / t_{7}, t_{2} / t_{1}, t_{2} / t_{8}
\end{array} ;, q\right)+\left(t_{2} \leftrightarrow t_{7}\right)
$$

as meromorphic functions in $t \in \mathcal{H}_{1}$.

(b) We have

$$
V_{t}(t)=\frac{2}{\left(q, t_{1}^{2} ; q\right)_{\infty}} \prod_{j=2}^{6} \frac{\left(t_{1} / t_{j} ; q\right)_{\infty}}{\left(t_{1} t_{j} ; q\right)_{\infty}}{ }_{8} W_{7}\left(\frac{t_{1}^{2}}{q} ; t_{1} t_{2}, t_{1} t_{3}, \ldots, t_{1} t_{6} ; q, \frac{1}{t_{1} t_{2} t_{3} t_{4} t_{5} t_{6}}\right)
$$

as meromorphic functions in $t \in\left(\mathbb{C}^{\times}\right)^{6} / C_{2}:\left|t_{1} t_{2} t_{3} t_{4} t_{5} t_{6}\right|>1$. 
Proof. This follows by a straightforward residue computation as in the proof of Lemma 5.5 (cf. also [5, §4.10]). For (a) one picks up the residues at the increasing pole sequences $t_{2} q^{\mathbb{Z}_{\geq 0}}$ and $t_{7} q^{\mathbb{Z}_{\geq 0}}$ of the integrand of $B_{t}(t)$; for (b) one picks up the residues at the single increasing pole sequence $t_{1} q^{\mathbb{Z}_{\geq 0}}$ of the integrand of $V_{t}(t)$.

Proposition 5.18. For generic $t \in \mathcal{H}_{1}$ we have

$$
\begin{aligned}
& \lim _{u \rightarrow 0} S_{t}\left(t_{1}, \ldots, t_{6}, t_{7} u, t_{8} / u\right)=E_{t}\left(t_{1}, \ldots, t_{6}\right), \\
& \lim _{u \rightarrow 0}\left(t_{2} t_{7} / u ; q\right)_{\infty} S_{t}\left(t_{1} u^{-\frac{1}{2}}, t_{2} u^{-\frac{1}{2}}, t_{3} u^{\frac{1}{2}}, t_{4} u^{\frac{1}{2}}, t_{5} u^{\frac{1}{2}}, t_{6} u^{\frac{1}{2}}, t_{7} u^{-\frac{1}{2}}, t_{8} u^{-\frac{1}{2}}\right)=B_{t}(t), \\
& \lim _{u \rightarrow 0}\left(t_{1} t_{7} / u ; q\right)_{\infty} U_{t}\left(t_{1}, \ldots, t_{6}, t_{7} / u, t_{8} u\right)=V_{t}\left(t_{1}, \ldots, t_{6}\right), \\
& \lim _{u \rightarrow 0} U_{t}\left(t_{2} u^{\frac{1}{2}}, t_{1} u^{\frac{1}{2}}, t_{3} u^{-\frac{1}{2}}, t_{4} u^{-\frac{1}{2}}, t_{5} u^{-\frac{1}{2}}, t_{6} u^{-\frac{1}{2}}, t_{8} u^{\frac{1}{2}}, t_{7} u^{\frac{1}{2}}\right)=B_{t}(t) .
\end{aligned}
$$

Proof. The first limit is direct. For the second limit, we follow the same approach as in the proof of Proposition 5.7. Define $Q(z)$ as

$$
Q(z)=\frac{\theta\left(t_{2} z s^{-\frac{1}{2}}, t_{7} z s^{-\frac{1}{2}}, \mu z s^{\frac{1}{2}}, t_{2} t_{7} \mu s^{-\frac{1}{2}} z^{-1} ; q\right)}{\theta\left(z^{2} ; q\right)} .
$$

Using (3.6) we obtain the equation

$$
Q(z)+Q\left(z^{-1}\right)=\theta\left(t_{2} t_{7} / s, t_{2} \mu, t_{7} \mu ; q\right),
$$

and hence, as in the proof of Proposition 5.7,

$$
\left(t_{2} t_{7} / u ; q\right)_{\infty} S_{t}\left(t_{u}\right)=2 \frac{\left(t_{2} t_{7} / u ; q\right)_{\infty}}{\theta\left(t_{2} t_{7} / u, t_{2} \mu, t_{7} \mu ; q\right)} \int_{\mathcal{C}} I_{t}\left(t_{u} ; z\right) Q(z) \frac{d z}{2 \pi i z}
$$

for an appropriate contour $\mathcal{C}$, where we use the abbreviated notation

$$
t_{u}=\left(t_{1} u^{-\frac{1}{2}}, t_{2} u^{-\frac{1}{2}}, t_{3} u^{\frac{1}{2}}, t_{4} u^{\frac{1}{2}}, t_{5} u^{\frac{1}{2}}, t_{6} u^{\frac{1}{2}}, t_{7} u^{-\frac{1}{2}}, t_{8} u^{-\frac{1}{2}}\right)
$$

Taking $u^{-\frac{1}{2}} z$ as a new integration variable we obtain

$$
\begin{aligned}
\left(u t_{2} t_{7} ; q\right)_{\infty} S_{t}\left(t_{u}\right)= & 2 \int_{\mathcal{C}} \frac{\theta\left(\mu z, t_{2} t_{7} \mu / z ; q\right)}{\theta\left(t_{2} \mu, t_{7} \mu ; q\right)} \frac{\left(z / t_{1}, z / t_{8} ; q\right)_{\infty}}{\prod_{j=3}^{6}\left(t_{j} z ; q\right)_{\infty}\left(t_{2} / z, t_{7} / z ; q\right)_{\infty}} \\
& \times\left(1-\frac{u}{z^{2}}\right) \frac{\left(u t_{1}^{-1} / z, u t_{8}^{-1} / z, q u t_{2}^{-1} / z, q u t_{7}^{-1} / z ; q\right)_{\infty}}{\left(q u / t_{2} t_{7} ; q\right)_{\infty} \prod_{j=3}^{6}\left(t_{j} u / z ; q\right)_{\infty}} \frac{d z}{2 \pi i z}
\end{aligned}
$$

where, for $u$ small enough, we take $\mathcal{C}$ to be a $u$-independent deformation of $\mathbb{T}$ separating the decreasing pole sequences $t_{2} q^{\mathbb{Z}_{\geq 0}}, t_{7} q^{\mathbb{Z}_{\geq 0}}$ and $t_{j} u q^{\mathbb{Z}_{\geq 0}}(j=3, \ldots, 6)$ of the integrand from the decreasing pole sequences $t_{j}^{-1} q^{\mathbb{Z}_{\leq 0}}(j=3, \ldots, 6)$. The limit $u \rightarrow 0$ can be taken in the resulting integral, leading to the desired result. 
To prove the third limit, we set $\mu=q / t_{1} t_{7} t_{8}$ in the integral expression of $U_{t}(t)=$ $\int_{\mathcal{C}^{\prime}} J_{t}^{\mu}(t ; z) \frac{d z}{2 \pi i z}$ to remove the contribution $\left(t_{7} z ; q\right)_{\infty}$ in the denominator of the integrand:

$$
\begin{aligned}
U_{t}(t)=2 & \int_{\mathcal{C}^{\prime}} \frac{\theta\left(t_{1} t_{7} t_{8} / z ; q\right)}{\theta\left(t_{1} t_{7}, t_{7} t_{8} ; q\right)}\left(1-\frac{z^{2}}{q}\right) \\
& \times \prod_{j=2}^{6} \frac{\left(z / t_{j} ; q\right)_{\infty}}{\left(t_{j} z ; q\right)_{\infty}} \frac{\left(z / t_{7}, q / t_{7} z ; q\right)_{\infty}}{\left(t_{1} z / q, t_{1} / z, t_{8} z / q, t_{8} / z ; q\right)_{\infty}} \frac{d z}{2 \pi i z} .
\end{aligned}
$$

In the resulting integral the desired limit can be taken directly, leading to the desired result.

For the fourth limit, one easily verifies that

$$
B_{t}(t)=\lim _{u \rightarrow 0} U_{t}^{\mu u^{\frac{1}{2}}}\left(t_{2} u^{\frac{1}{2}}, t_{1} u^{\frac{1}{2}}, t_{3} u^{-\frac{1}{2}}, t_{4} u^{-\frac{1}{2}}, t_{5} u^{-\frac{1}{2}}, t_{6} u^{-\frac{1}{2}}, t_{8} u^{\frac{1}{2}}, t_{7} u^{\frac{1}{2}}\right)
$$

for generic $t \in \mathcal{H}_{1}$ after changing the integration variable $z$ to $z u^{\frac{1}{2}}$ on the right-hand side.

Proposition 5.18 and Corollary 5.9 immediately lead to the following three trigonometric integral evaluations (of which the first is the well known Askey-Wilson integral evaluation $[5,(6.1 .4)])$.

Corollary 5.19. For generic parameters $t=\left(t_{1}, t_{2}, t_{3}, t_{4}\right) \in \mathbb{C}^{4}$ we have

$$
\begin{aligned}
\int_{\mathcal{C}} \frac{\left(z^{ \pm 2} ; q\right)_{\infty}}{\prod_{j=1}^{4}\left(t_{j} z^{ \pm 1} ; q\right)_{\infty}} \frac{d z}{2 \pi i z} & =\frac{2\left(t_{1} t_{2} t_{3} t_{4} ; q\right)_{\infty}}{(q ; q)_{\infty} \prod_{1 \leq j<k \leq 4}\left(t_{j} t_{k} ; q\right)_{\infty}} \\
\int_{\mathcal{C}^{\prime}} \frac{\theta\left(q t_{2} t_{3} t_{4} z ; q\right)}{\theta\left(q t_{1} t_{2} t_{3} t_{4} ; q\right)}\left(1-\frac{z^{2}}{q}\right) & \frac{\left(z / t_{2}, z / t_{3}, z / t_{4} ; q\right)_{\infty}}{\left(t_{1} / z, t_{1} z / q, t_{2} z, t_{3} z, t_{4} z ; q\right)_{\infty}} \frac{d z}{2 \pi i z} \\
& =\frac{\left(q t_{1} t_{2} t_{3} t_{4}, 1 / t_{2} t_{3}, 1 / t_{2} t_{4}, 1 / t_{3} t_{4} ; q\right)_{\infty}}{\left(q, t_{1} t_{2}, t_{1} t_{3}, t_{1} t_{4} ; q\right)_{\infty}}
\end{aligned}
$$

with $\mathcal{C}$ (respectively $\mathcal{C}^{\prime}$ ) a deformation of $\mathbb{T}$ separating the sequences $t_{j} q^{\mathbb{Z}} \geq 0 \quad(j=$ $1, \ldots, 4$ ) from their reciprocals (respectively separating $t_{1} q^{\mathbb{Z}_{\geq 0}}$ from $t_{1}^{-1} q^{\mathbb{Z}_{\leq 1}}, t_{2}^{-1} q^{\mathbb{Z}_{\leq 0}}$, $t_{3}^{-1} q^{\mathbb{Z}_{\leq 0}}$ and $\left.t_{4}^{-1} q^{\mathbb{Z}_{\leq 0}}\right)$.

For generic $\mu \in \mathbb{C}^{\times}$and $t \in \mathbb{C}^{6}$ satisfying $\prod_{j=1}^{6} t_{j}=1$ we have

$$
\begin{gathered}
\int_{\mathcal{C}} \frac{\theta\left(t_{1} t_{5} / \mu z, z / \mu ; q\right)}{\theta\left(t_{1} / \mu, t_{5} / \mu ; q\right)} \frac{\left(z / t_{6} ; q\right)_{\infty}}{\left(t_{1} / z, t_{2} z, t_{3} z, t_{4} z, t_{5} / z ; q\right)_{\infty}} \frac{d z}{2 \pi i z} \\
=\frac{1}{(q ; q)_{\infty}} \prod_{j=2}^{4} \frac{\left(1 / t_{j} t_{6} ; q\right)_{\infty}}{\left(t_{1} t_{j}, t_{j} t_{5} ; q\right)_{\infty}},
\end{gathered}
$$

with $\mathcal{C}$ a deformation of $\mathbb{T}$ separating the pole sequences $t_{1} q^{\mathbb{Z}_{\geq 0}}, t_{5} q^{\mathbb{Z}_{\geq 0}}$ from $t_{2}^{-1} q^{\mathbb{Z}_{\leq 0}}$, $t_{3}^{-1} q^{\mathbb{Z}_{\leq 0}}$ and $t_{4}^{-1} q^{\mathbb{Z}_{\leq 0}}$. 
Proof. Specializing the degeneration from $S_{t}$ to $E_{t}$ in Proposition 5.18 to generic parameters $t \in \mathcal{H}_{1}$ under the additional condition $t_{1} t_{2}=1$ and using the trigonometric Nassrallah-Rahman integral evaluation (Corollary 5.9) leads to the Askey-Wilson integral evaluation with corresponding parameters $\left(t_{3}, t_{4}, t_{5}, t_{6}\right)$.

Similarly, specializing the degeneration from $U_{t}$ to $V_{t}$ (respectively $S_{t}$ to $B_{t}$ ) to generic parameters $t \in \mathcal{H}_{1}$ under the additional condition $t_{2} t_{3}=1$ (respectively $t_{1} t_{3}=1$ ) and using the Nassrallah-Rahman integral evaluation we obtain the second (respectively third) integral evaluation with parameters $\left(t_{1}, t_{4}, t_{5}, t_{6}\right)$ (respectively $\left.\left(t_{2}, t_{4}, t_{5}, t_{6}, t_{7}, t_{8}\right)\right)$.

Various well-known identities are direct consequences of Corollary 5.19. Firstly, analogous to the unfolding of the integrals $U_{t}$ and $V_{t}$ (see Corollary 5.3 and Lemma 5.16), the left-hand side of the third integral evaluation can be unfolded. We obtain for generic $u \in \mathbb{C}^{6}$ with $\sum_{j=1}^{6} u_{j}=0$,

$$
\begin{aligned}
& \int_{\mathcal{L}} \frac{\left(q e\left(x-u_{1}\right), q e\left(x-u_{5}\right), e\left(x-u_{6}\right) ; q\right)_{\infty}}{\left(e\left(x+u_{2}\right), e\left(x+u_{3}\right), e\left(x+u_{4}\right) ; q\right)_{\infty}} \frac{e(x)}{\left(1-e\left(\left(u_{5}-x\right) / \tau\right)\right)\left(1-e\left(\left(x-u_{1}\right) / \tau\right)\right)} d x \\
& =\frac{\tau t_{1} \theta\left(t_{5} / t_{1} ; q\right)}{\left(e\left(\left(u_{5}-u_{1}\right) / \tau\right)-1\right)} \frac{\left(q, 1 / t_{2} t_{6}, 1 / t_{3} t_{6}, 1 / t_{4} t_{6} ; q\right)_{\infty}}{\left(t_{1} t_{2}, t_{1} t_{3}, t_{1} t_{4}, t_{2} t_{5}, t_{3} t_{5}, t_{4} t_{5} ; q\right)_{\infty}},
\end{aligned}
$$

where $\tau \in \mathbb{H}_{+}$such that $q=e(\tau)$, where $t_{j}=e\left(u_{j}\right)(j=1, \ldots, 6)$ and where the integration contour $\mathcal{L}$ is some translate $\xi+\mathbb{R}(\xi \in i \mathbb{R})$ of the real line with a finite number of indentations such that $\mathcal{C}$ separates the pole sequences $-u_{j}+\mathbb{Z}+\mathbb{Z} \leq 0 \tau(j=2,3,4)$ of the integrand from the pole sequences $u_{1}+\mathbb{Z}_{\geq 0} \tau$ and $u_{5}+\mathbb{Z}_{\geq 0} \tau$. This integral identity is Agarwal's [5, (4.4.6)] trigonometric analogue of Barnes' second lemma.

The left-hand side of the second integral evaluation in Corollary 5.19 can be rewritten as a unilateral sum by picking up the residues at $t_{1} q^{\mathbb{Z}_{\geq 0}}$, cf. Lemma 5.5. The resulting identity is

$$
{ }_{6} \phi_{5}\left(\begin{array}{l}
q^{-1} t_{1}^{2}, q^{\frac{1}{2}} t_{1},-q^{\frac{1}{2}} t_{1}, t_{1} t_{2}, t_{1} t_{3}, t_{1} t_{4} \\
q^{-\frac{1}{2}} t_{1},-q^{-\frac{1}{2}} t_{1}, t_{1} / t_{2}, t_{1} / t_{3}, t_{1} / t_{4}
\end{array} ; \frac{1}{t_{1} t_{2} t_{3} t_{4}}\right)=\frac{\left(t_{1}^{2}, 1 / t_{2} t_{3}, 1 / t_{2} t_{4}, 1 / t_{3} t_{4} ; q\right)_{\infty}}{\left(1 / t_{1} t_{2} t_{3} t_{4}, t_{1} / t_{2}, t_{1} / t_{3}, t_{1} / t_{4} ; q\right)_{\infty}},
$$

for generic $t \in \mathbb{C}^{4}$ satisfying $\left|t_{1} t_{2} t_{3} t_{4}\right|>1$, which is the ${ }_{6} \phi_{5}$ summation formula [5, (2.7.1)].

For generic $t \in \mathbb{C}^{6}$ satisfying $\prod_{j=1}^{6} t_{j}=1$ the left-hand side of the third integral evaluation in Corollary 5.19 can be written as a sum of two unilateral series by picking up the poles of the integrand at the decreasing sequences $t_{1} q^{\mathbb{Z}_{\geq 0}}$ and $t_{5} q^{\mathbb{Z}_{\geq 0}}$ of poles of the integrand. The resulting identity is

$$
\frac{\left(t_{1} / t_{6} ; q\right)_{\infty}}{\left(t_{5} / t_{1}, t_{1} t_{2}, t_{1} t_{3}, t_{1} t_{4} ; q\right)_{\infty}} 3 \phi_{2}\left(\begin{array}{c}
t_{1} t_{2}, t_{1} t_{3}, t_{1} t_{4} \\
q t_{1} / t_{5}, t_{1} / t_{6}
\end{array} q, q\right)+\left(t_{1} \leftrightarrow t_{5}\right)=\prod_{j=2}^{4} \frac{\left(1 / t_{j} t_{6} ; q\right)_{\infty}}{\left(t_{1} t_{j}, t_{j} t_{5} ; q\right)_{\infty}}
$$

for generic $t \in \mathbb{C}^{6}$ satisfying $\prod_{j=1}^{6} t_{j}=1$, which is the nonterminating version [5, (2.10.12)] of Saalschütz formula.

We now return to the three trigonometric hypergeometric integrals $E_{t}, B_{t}$ and $V_{t}$. Recall that the symmetry group of $S_{t}$ and $U_{t}$ is the subgroup $W\left(E_{6}\right)=W\left(E_{7}\right)_{\alpha_{18}^{+}}=$ $W\left(E_{7}\right)_{\gamma_{18}}$, which is a maximal standard parabolic subgroup of $W\left(E_{7}\right)$ with respect to both bases $\Delta_{1}$ and $\Delta_{2}$ of $R\left(E_{7}\right)$ (see Sect. 2), with corresponding sub-bases $\Delta_{1}^{\prime}=$ $\Delta_{1} \backslash\left\{\alpha_{12}^{-}\right\}$and $\Delta_{2}^{\prime}=\Delta_{2} \backslash\left\{\alpha_{87}^{-}\right\}$respectively. The four limits of Proposition 5.18 now 
imply that the trigonometric integrals $E_{t}, B_{t}$ and $V_{t}$ have symmetry groups $W\left(E_{6}\right)_{\alpha_{78}^{-}}$ or $W\left(E_{6}\right)_{\beta_{1278}}$. The stabilizer subgroup $W\left(E_{6}\right)_{\alpha_{78}^{-}}$is a standard maximal parabolic subgroup of $W\left(E_{6}\right)$ with respect to both bases $\Delta_{1}^{\prime}$ or $\Delta_{2}^{\prime}$, with corresponding sub-basis

$$
\Delta\left(D_{5}\right)=\Delta_{1}^{\prime} \backslash\left\{\alpha_{76}^{-}\right\}=\Delta_{2}^{\prime} \backslash\left\{\alpha_{18}^{-}\right\}
$$

and with corresponding Dynkin sub-diagrams
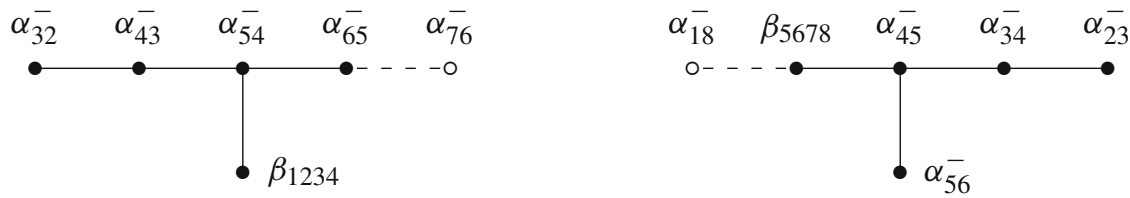

respectively. Similarly, $W\left(E_{6}\right)_{\beta_{1278}}$ is a standard maximal parabolic subgroup of $W\left(E_{6}\right)$ with respect to the basis $\Delta_{2}^{\prime}$, with corresponding sub-basis

$$
\Delta^{\prime}\left(D_{5}\right)=\Delta_{2}^{\prime} \backslash\left\{\alpha_{23}^{-}\right\}
$$

and with corresponding Dynkin sub-diagram

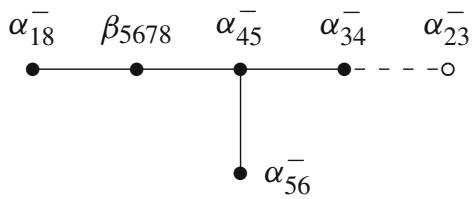

We write

$$
W\left(D_{5}\right)=W\left(E_{6}\right)_{\alpha_{78}^{-}}, \quad W^{\prime}\left(D_{5}\right)=W\left(E_{6}\right)_{\beta_{1278}}
$$

for the corresponding isotropy group, which are both isomorphic to the Weyl group of type $D_{5}$.

The isotropy group $W\left(D_{5}\right)$ acts on $\left(\mathbb{C}^{\times}\right)^{6} / C_{2}$ : the simple reflections corresponding to roots of the form $\alpha_{i j}^{-} \in \Delta\left(D_{5}\right)$ act by permuting the $i^{\text {th }}$ and $j^{\text {th }}$ coordinate, while $w$ acts by

$$
w( \pm t)= \pm\left(s t_{1}, s t_{2}, s t_{3}, s t_{4}, t_{5} / s, t_{6} / s\right), \quad s^{2}=1 / t_{1} t_{2} t_{3} t_{4} .
$$

With this action, the degenerations to $E_{t}$ and $V_{t}$ in Proposition 5.18 are $W\left(D_{5}\right)$-equivariant in an obvious sense.

We can now directly compute the $W\left(D_{5}\right)$-symmetries of the trigonometric integrals $E_{t}$ and $V_{t}$, as well as $W^{\prime}\left(D_{5}\right)$-symmetries of $B_{t}$, by taking limits of the corresponding symmetries for $S_{t}$ and $U_{t}$ using Proposition 5.18. This yields the following result.

Proposition 5.20. a) The trigonometric hypergeometric integrals $E_{t}(t)$ and $V_{t}(t)$ $\left(t \in\left(\mathbb{C}^{\times}\right)^{6} / C_{2}\right)$ are invariant under permutations of $\left(t_{2}, \ldots, t_{6}\right)$. Furthermore,

$$
\begin{aligned}
& E_{t}(t)=E_{t}(w t) \frac{\left(1 / t_{1} t_{2}, 1 / t_{1} t_{3}, 1 / t_{1} t_{4}, t_{1} t_{2} t_{3} t_{4} t_{5} t_{6} ; q\right)_{\infty}}{\left(t_{2} t_{3}, t_{2} t_{4}, t_{3} t_{4}, t_{5} t_{6} ; q\right)_{\infty}}, \\
& V_{t}(t)=V_{t}(w t) \frac{\left(1 / t_{2} t_{3}, 1 / t_{2} t_{4}, 1 / t_{3} t_{4}, 1 / t_{5} t_{6} ; q\right)_{\infty}}{\left(t_{1} t_{2}, t_{1} t_{3}, t_{1} t_{4}, 1 / t_{1} t_{2} t_{3} t_{4} t_{5} t_{6} ; q\right)_{\infty}} .
\end{aligned}
$$

as meromorphic functions in $t \in\left(\mathbb{C}^{\times}\right)^{6} / C_{2}$. 
b) The trigonometric Barnes integral $B_{t}(t)\left(t \in \mathcal{H}_{1}\right)$ is invariant under permutations of the pairs $\left(t_{1}, t_{8}\right),\left(t_{2}, t_{7}\right)$ and of $\left(t_{3}, t_{4}, t_{5}, t_{6}\right)$. Furthermore,

$$
B_{t}(t)=B_{t}(w t) \frac{\left(1 / t_{1} t_{3}, 1 / t_{1} t_{4}, 1 / t_{5} t_{8}, 1 / t_{6} t_{8} ; q\right)_{\infty}}{\left(t_{2} t_{4}, t_{2} t_{3}, t_{5} t_{7}, t_{6} t_{7} ; q\right)_{\infty}}
$$

as meromorphic functions in $t \in \mathcal{H}_{1}$.

Remark 5.21. The $w$-symmetry of $V_{t}$, rewritten in series form using Lemma 5.17, gives the transformation formula [5, (2.10.1)] for very-well-poised ${ }_{8} \phi_{7}$ basic hypergeometric series.

Similarly as in the hyperbolic theory, the $w$-symmetry of $E_{t}$ generalizes to the following integral transformation formula for the trigonometric Euler integral $E_{t}$.

Proposition 5.22. For $t \in\left(\mathbb{C}^{\times}\right)^{6}$ and $s \in \mathbb{C}^{\times}$satisfying

$$
\left|t_{2}\right|,\left|t_{3}\right|,\left|t_{4}\right|,|s|,\left|t_{5} / s\right|,\left|t_{6} / s\right|<1
$$

we have

$$
\begin{aligned}
\int_{\mathbb{T}} & E_{t}\left(t_{1}, t_{2}, t_{3}, t_{4}, s x, s x^{-1}\right) \frac{\left(x^{ \pm 2} ; q\right)_{\infty}}{\left(t_{5} x^{ \pm 1} / s, t_{6} x^{ \pm 1} / s ; q\right)_{\infty}} \frac{d x}{2 \pi i x} \\
= & \frac{2\left(t_{5} t_{6} ; q\right)_{\infty}}{\left(q, s^{2}, t_{5} t_{6} / s^{2} ; q\right)_{\infty}} E_{t}\left(t_{1}, \ldots, t_{6}\right) .
\end{aligned}
$$

Proof. The proof is similar to the hyperbolic case (see Proposition 4.13), now using the double integral

$$
\int_{\mathbb{T}^{2}} \frac{\left(z^{ \pm 2}, z^{ \pm 1} / t_{1}, x^{ \pm 2} ; q\right)_{\infty}}{\left(s z^{ \pm 1} x^{ \pm 1} ; q\right)_{\infty} \prod_{j=2}^{4}\left(t_{j} z^{ \pm 1} ; q\right)_{\infty} \prod_{k=5}^{6}\left(t_{k} x^{ \pm 1} / s ; q\right)_{\infty}} \frac{d z}{2 \pi i z} \frac{d x}{2 \pi i x} .
$$

Specializing $s^{2}=1 / t_{1} t_{2} t_{3} t_{4}$ in Proposition 5.22 and using the trigonometric Nassrallah-Rahman integral (see Corollary 5.19), we re-obtain the $w$-symmetry of $E_{t}$ (see Proposition 5.20).

The three trigonometric integrals $E_{t}, B_{t}$ and $V_{t}$ are interconnected as follows.

Proposition 5.23. We have

$$
\begin{aligned}
B_{t}(t) & =\frac{\left(1 / t_{1} t_{6}, 1 / t_{8} t_{6} ; q\right)_{\infty}}{\left(t_{2} t_{3}, t_{2} t_{4}, t_{2} t_{5}, t_{6} t_{7} ; q\right)_{\infty}} V_{t}\left(t_{7} / s, t_{3} s, t_{4} s, t_{5} s, t_{1} / s, t_{8} / s\right), \\
& =\frac{\left(1 / t_{1} t_{3}, 1 / t_{1} t_{4}, 1 / t_{1} t_{5}, 1 / t_{6} t_{8} ; q\right)_{\infty}}{\left(t_{2} t_{6}, t_{7} t_{6} ; q\right)_{\infty}} E_{t}\left(t_{8} / v, t_{7} / v, t_{3} v, t_{4} v, t_{5} v, t_{2} / v\right),
\end{aligned}
$$

as meromorphic functions in $t \in \mathcal{H}_{1}$, where $s^{2}=t_{1} t_{6} t_{7} t_{8}=1 / t_{2} t_{3} t_{4} t_{5}$ and $v^{2}=t_{2} t_{6} t_{7} t_{8}=1 / t_{1} t_{3} t_{4} t_{5}$. 
Proof. This follows by combining Proposition 5.12 and Proposition 5.18. Concretely, to relate $B_{t}$ and $V_{t}$ one computes for generic $t \in \mathcal{H}_{1}$ and with $s^{2}=1 / t_{2} t_{3} t_{4} t_{5}$,

$$
\begin{aligned}
B_{t}(t)= & \lim _{u \rightarrow 0}\left(t_{2} t_{7} / u ; q\right)_{\infty} S_{t}\left(t_{1} u^{-\frac{1}{2}}, t_{2} u^{-\frac{1}{2}}, t_{3} u^{\frac{1}{2}}, t_{4} u^{\frac{1}{2}}, t_{5} u^{\frac{1}{2}}, t_{6} u^{\frac{1}{2}}, t_{7} u^{-\frac{1}{2}}, t_{8} u^{-\frac{1}{2}}\right) \\
= & \frac{\left(1 / t_{1} t_{6}, 1 / t_{8} t_{6} ; q\right)_{\infty}}{\left(t_{2} t_{3}, t_{2} t_{4}, t_{2} t_{5}, t_{6} t_{7} ; q\right)_{\infty}} \\
& \times \lim _{u \rightarrow 0}\left(t_{2} t_{7} / u ; q\right)_{\infty} U_{t}\left(t_{7} / s, t_{3} s, t_{4} s, t_{5} s, t_{1} / s, t_{8} / s, t_{2} s / u, t_{6} u / s\right) \\
= & \frac{\left(1 / t_{1} t_{6}, 1 / t_{8} t_{6} ; q\right)_{\infty}}{\left(t_{2} t_{3}, t_{2} t_{4}, t_{2} t_{5}, t_{6} t_{7} ; q\right)_{\infty}} V_{t}\left(t_{7} / s, t_{3} s, t_{4} s, t_{5} s, t_{1} / s, t_{8} / s\right)
\end{aligned}
$$

where the first and third equality follows from Proposition 5.18 and the second equality follows from Proposition 5.12. To relate $B_{t}$ and $E_{t}$, we first note that Proposition 5.12 is equivalent to the identity

$$
U_{t}(t)=\frac{\left(1 / t_{6} t_{7} ; q\right)_{\infty} \prod_{2 \leq j<k \leq 5}\left(1 / t_{j} t_{k} ; q\right)_{\infty}}{\left(t_{1} t_{8} / q, t_{1} t_{6}, t_{8} t_{6}, t_{1} t_{7}, t_{8} t_{7} ; q\right)_{\infty}} S_{t}\left(t_{6} / s, t_{2} s, t_{3} s, t_{4} s, t_{5} s, t_{1} / s, t_{8} / s, t_{7} / s\right),
$$

where $t \in \mathcal{H}_{1}$ and $s^{2}=1 / t_{2} t_{3} t_{4} t_{5}$. For generic $t \in \mathcal{H}_{1}$ and with $v^{2}=1 / t_{1} t_{3} t_{4} t_{5}$ we then compute

$$
\begin{aligned}
B_{t}(t)= & \lim _{u \rightarrow 0} U_{t}\left(t_{2} u^{\frac{1}{2}}, t_{1} u^{\frac{1}{2}}, t_{3} u^{-\frac{1}{2}}, t_{4} u^{-\frac{1}{2}}, t_{5} u^{-\frac{1}{2}}, t_{6} u^{-\frac{1}{2}}, t_{8} u^{\frac{1}{2}}, t_{7} u^{\frac{1}{2}}\right) \\
= & \frac{\left(1 / t_{1} t_{3}, 1 / t_{1} t_{4}, 1 / t_{1} t_{5}, 1 / t_{6} t_{8} ; q\right)_{\infty}}{\left(t_{2} t_{6}, t_{7} t_{6} ; q\right)_{\infty}} \\
& \times \lim _{u \rightarrow 0} S_{t}\left(t_{8} / v, t_{7} / v, t_{3} v, t_{4} v, t_{5} v, t_{2} / v, t_{1} v u, t_{6} / v u\right) \\
= & \frac{\left(1 / t_{1} t_{3}, 1 / t_{1} t_{4}, 1 / t_{1} t_{5}, 1 / t_{6} t_{8} ; q\right)_{\infty}}{\left(t_{2} t_{6}, t_{7} t_{6} ; q\right)_{\infty}} E_{t}\left(t_{8} / v, t_{7} / v, t_{3} v, t_{4} v, t_{5} v, t_{2} / v\right),
\end{aligned}
$$

where the first and third equality follows from Proposition 5.18 and the second equality follows from (5.16).

Remark 5.24. a) Combining the interconnection between $E_{t}$ and $V_{t}$ from Proposition 5.23 with the expression of $V_{t}$ as a very-well-poised ${ }_{8} \phi_{7}$ series from Lemma 5.17 yields the Nassrallah-Rahman integral representation [5, (6.3.7)].

b) Similarly, combining the interconnection between $B_{t}$ and $V_{t}$ from Proposition 5.23 with their series expressions from Lemma 5.17 yields the expression [5, (2.10.10)] of a very-well-poised ${ }_{8} \phi_{7}$ series as a sum of two balanced ${ }_{4} \phi_{3}$ series.

Degenerating the contiguous relations of $S_{t}$ using Proposition 5.18 leads directly to contiguous relations for $E_{t}, B_{t}$ and $V_{t}$. For instance, we obtain

Proposition 5.25. We have

$$
A(t) E_{t}\left(t_{1}, t_{2}, t_{3}, q t_{4}, t_{5} / q, t_{6}\right)+\left(t_{4} \leftrightarrow t_{5}\right)=B(t) E_{t}(t)
$$


as meromorphic functions in $t \in\left(\mathbb{C}^{\times}\right)^{6} / C_{2}$, where

$$
\begin{aligned}
& A(t)=-\frac{\left(1-\frac{1}{t_{1} t_{5}}\right)\left(1-\frac{t_{2} t_{5}}{q}\right)\left(1-\frac{t_{3} t_{5}}{q}\right)\left(1-\frac{t_{6} t_{5}}{q}\right)}{t_{4}\left(1-\frac{t_{4} t_{5}}{q}\right)\left(1-\frac{t_{5}}{q t_{4}}\right)\left(1-\frac{t_{4}}{t_{5}}\right)}, \\
& B(t)=\frac{q}{t_{1} t_{4} t_{5}}-t_{2} t_{3} t_{6}+\frac{q t_{4}}{t_{5}} A(t)+\frac{q t_{5}}{t_{4}} A\left(s_{45} t\right) .
\end{aligned}
$$

Proof. Substitute $t=\left(t_{1}, t_{2}, t_{3}, t_{4}, t_{5}, t_{7} u, t_{6}, t_{8} / u\right)$ with $\prod_{j=1}^{8} t_{j}=1$ in (5.11) and take the limit $u \rightarrow 0$.

For later purposes, we also formulate the corresponding result for $B_{t}(t)$. We substitute $t=\left(t_{1} u^{-\frac{1}{2}}, t_{7} u^{-\frac{1}{2}}, t_{3} u^{\frac{1}{2}}, t_{4} u^{\frac{1}{2}}, t_{5} u^{\frac{1}{2}}, t_{2} u^{-\frac{1}{2}}, t_{6} u^{\frac{1}{2}}, t_{8} u^{-\frac{1}{2}}\right)$ for generic $t \in \mathbb{C}^{8}$ satisfying $\prod_{j=1}^{8} t_{j}=1$ in (5.11), multiply the resulting equation by $u^{\frac{1}{2}}\left(t_{2} t_{7} / u ; q\right)_{\infty}$, and take the limit $u \rightarrow 0$. We arrive at

$$
\alpha(t) B_{t}\left(t_{1}, t_{2}, t_{3}, q t_{4}, t_{5} / q, t_{6}, t_{7}, t_{8}\right)+\left(t_{4} \leftrightarrow t_{5}\right)=\beta(t) B_{t}(t), \quad t \in \mathcal{H}_{1},
$$

where

$$
\begin{aligned}
& \alpha(t)=-\frac{\left(1-\frac{1}{t_{1} t_{5}}\right)\left(1-\frac{t_{2} t_{5}}{q}\right)\left(1-\frac{t_{5} t_{7}}{q}\right)\left(1-\frac{1}{t_{5} t_{8}}\right)}{t_{4}\left(1-\frac{t_{4}}{t_{5}}\right)\left(1-\frac{t_{5}}{q t_{4}}\right)}, \\
& \beta(t)=t_{7}\left(1-t_{2} t_{3}\right)\left(1-t_{2} t_{6}\right)+\frac{\left(1-t_{2} t_{4}\right)}{\left(1-\frac{t_{2} t_{5}}{q}\right)} \alpha(t)+\frac{\left(1-\frac{t_{2} t_{4}}{q}\right)}{\left(1-t_{2} t_{5}\right)} \alpha\left(s_{45} t\right) .
\end{aligned}
$$

The degeneration of (5.12) yields Bailey's [5, (2.11.1)] three term transformation formula for very-well-poised ${ }_{8} \phi_{7}$ 's:

Proposition 5.26. We have

$$
\frac{\theta\left(t_{1}^{-1} t_{2}^{ \pm 1} ; q\right)}{\theta\left(t_{3} t_{2}^{ \pm 1} ; q\right)} E_{t}\left(t_{3} / q, q t_{1}, t_{2}, t_{4}, t_{5}, t_{6}\right)+\left(t_{2} \leftrightarrow t_{3}\right)=E_{t}(t) .
$$

Proof. Consider (5.12) with $t_{1}$ and $t_{8}, t_{2}$ and $t_{7}$, and $t_{3}$ and $t_{6}$ interchanged. Subsequently substitute the parameters $\left(t_{1}, t_{2}, t_{3}, t_{4}, t_{5}, t_{6}, t_{7} u, t_{8} / u\right)$ with $\prod_{j=1}^{8} t_{j}=1$ and take the limit $u \rightarrow 0$.

5.5. The Askey-Wilson function. In this subsection we relate the trigonometric hypergeometric integrals with $D_{5}$ symmetry to the nonpolynomial eigenfunction of the Askey-Wilson second order difference operator, known as the Askey-Wilson function. The Askey-Wilson function is the trigonometric analog of Ruijsenaars' $R$-function, and is closely related to harmonic analysis on the quantum $\mathrm{SU}(1,1)$ group.

As for the $R$-function, we introduce the Askey-Wilson function in terms of the trigonometric Barnes integral $B_{t}$. Besides the usual Askey-Wilson parameters we also use logarithmic variables in order to make the connection to the $R$-function more transparent. We write the base $q \in \mathbb{C}^{\times}$with $|q|<1$ as $q=e\left(\omega_{1} / \omega_{2}\right)$ with $\tau=\omega_{1} / \omega_{2} \in \mathbb{H}_{+}$ and $e(x)=\exp (2 \pi i x)$ as before. From the previous subsection it follows that the parameter space of $B_{t}(t)$ is $\mathcal{H}_{1} / \mathbb{C}^{\times} e\left(\beta_{1278}\right)$. In logarithmic coordinates, this relates to 
$\mathcal{G}_{0} / \mathbb{C} \beta_{1278}$. We identify $\mathcal{G}_{0} / \mathbb{C} \beta_{1278}$ with $\mathbb{C}^{6}$ by assigning to the six-tuple $(\gamma, x, \lambda)=$ $\left(\gamma_{0}, \gamma_{1}, \gamma_{2}, \gamma_{3}, \lambda, x\right)$ the class in $\mathcal{G}_{0} / \mathbb{C} \beta_{1278}$ represented by $u=\left(u_{1}, \ldots, u_{8}\right) \in \mathcal{G}_{0}$ with

$$
\begin{array}{ll}
u_{1}=\left(-\gamma_{0}-\gamma_{1}-2 \omega\right) / \omega_{2}, & u_{2}=0, \\
u_{3}=\left(\hat{\gamma}_{0}+\omega-i \lambda\right) / \omega_{2}, & u_{4}=\left(\gamma_{0}+\omega-i x\right) / \omega_{2}, \\
u_{5}=\left(\gamma_{0}+\omega+i x\right) / \omega_{2}, & u_{6}=\left(\hat{\gamma}_{0}+\omega+i \lambda\right) / \omega_{2}, \\
u_{7}=\left(-\gamma_{0}-\gamma_{3}\right) / \omega_{2}, & u_{8}=\left(-\gamma_{0}-\gamma_{2}-2 \omega\right) / \omega_{2},
\end{array}
$$

where $\omega=\frac{1}{2}\left(\omega_{1}+\omega_{2}\right)$ as before. We define the corresponding six-tuple of Askey-Wilson parameters $(a, b, c, d, \mu, z)$ by

$$
\begin{aligned}
(a, b, c, d) & \left.=\left(e\left(\left(\gamma_{0}+\omega\right) / \omega_{2}\right), e\left(\left(\gamma_{1}+\omega\right) / \omega_{2}\right), e\left(\left(\gamma_{2}+\omega\right) / \omega_{2}\right), e\left(\gamma_{3}+\omega\right) / \omega_{2}\right)\right), \\
(\mu, z) & =\left(e\left(-i \lambda / \omega_{2}\right), e\left(-i x / \omega_{2}\right)\right) .
\end{aligned}
$$

The four-tuple $(a, b, c, d)$ represents the four parameter freedom in the AskeyWilson theory, while $z$ (respectively $\mu$ ) plays the role of geometric (respectively spectral) parameter. Furthermore, we define the dual Askey-Wilson parameters by

$$
(\widetilde{a}, \widetilde{b}, \widetilde{c}, \widetilde{d})=\left(e\left(\left(\hat{\gamma}_{0}+\omega\right) / \omega_{2}\right), e\left(\left(\hat{\gamma}_{1}+\omega\right) / \omega_{2}\right), e\left(\left(\hat{\gamma}_{2}+\omega\right) / \omega_{2}\right), e\left(\left(\hat{\gamma}_{3}+\omega\right) / \omega_{2}\right)\right),
$$

with $\hat{\gamma}$ the dual parameters defined by (4.29). We furthermore associate to the logarithmic parameters $u \in \mathcal{G}_{0}($ see $(5.18))$ the parameters $t=\psi_{0}(2 \pi i u) \in \mathcal{H}_{1}$, so that

$$
t=\psi_{0}(2 \pi i u)=(1 / a b, 1, \tilde{a} \mu, a z, a / z, \widetilde{a} / \mu, q / a d, 1 / a c) .
$$

We define the Askey-Wilson function $\phi(\gamma ; x, \lambda)=\phi\left(\gamma ; x, \lambda ; \omega_{1}, \omega_{2}\right)$ by

$$
\phi(\gamma ; x, \lambda)=\frac{\left(q, t_{2} t_{3}, t_{2} t_{4}, t_{2} t_{5}, t_{2} t_{6} ; q\right)_{\infty}}{2} B_{t}(t)
$$

with $t=\psi_{0}(2 \pi i u) \in \mathcal{H}_{1}$ and $u$ given by (5.18). Note the similarity to the definition of Ruijsenaars' $R$-function, see (4.27).

From the series expansion of $B_{t}$ as sum of two balanced ${ }_{4} \phi_{3}$, we have in terms of Askey-Wilson parameters (5.19),

$$
\begin{aligned}
\phi(\gamma ; x, \lambda)= & \frac{(a b, a c ; q)_{\infty}}{(q / a d ; q)_{\infty}}{ }_{4} \phi_{3}\left(\begin{array}{c}
a z^{ \pm 1}, \widetilde{a} \mu^{ \pm 1} \\
a b, a c, a d
\end{array} ; q, q\right) \\
& +\frac{\left(q b / d, q c / d, \widetilde{a} \mu^{ \pm 1}, a z^{ \pm 1} ; q\right)_{\infty}}{\left(a d / q, q \widetilde{a} \mu^{ \pm 1} / a d, q z^{ \pm 1} / d ; q\right)_{\infty}}{ }_{4} \phi_{3}\left(\begin{array}{l}
q z^{ \pm 1} / d, q \widetilde{a} \mu^{ \pm 1} / a d \\
q^{2} / a d, q b / d, q c / d
\end{array} ;, q\right),
\end{aligned}
$$

which shows that $\phi(\gamma ; x, \lambda)$ is, up to a $(z, \gamma)$-independent rescaling factor, the AskeyWilson function as defined in e.g. [11].

We now re-derive several fundamental properties of the Askey-Wilson function using the results of the previous subsection. Comparing the symmetries of the Askey-Wilson function $\phi(\gamma ; x, \lambda)$ to the symmetries of the $R$-function (Proposition 4.18), the symmetry in the parameters $\gamma$ is broken (from the Weyl group of type $D_{4}$ to the Weyl group of type $D_{3}$ ). The most important symmetry (self-duality) is also valid for the Askey-Wilson function and has played a fundamental role in the study of the associated generalized Fourier transform (see [11]). Self-duality of the Askey-Wilson function has a natural interpretation in terms of Cherednik's theory on double affine Hecke algebras, see [36]. Concretely, the symmetries of the Askey-Wilson function are as follows. 
Proposition 5.27. The Askey-Wilson function $\phi(\gamma ; x, \lambda)$ is even in $x$ and $\lambda$ and is selfdual,

$$
\phi(\gamma ; x, \lambda)=\phi(\gamma ;-x, \lambda)=\phi(\gamma ; x,-\lambda)=\phi(\hat{\gamma} ; \lambda, x) .
$$

Furthermore, $\phi(\gamma ; x, \lambda)$ has a $W\left(D_{3}\right)$-symmetry in the parameters $\gamma$, given by

$$
\begin{aligned}
\phi\left(\gamma_{1}, \gamma_{0}, \gamma_{2}, \gamma_{3} ; x, \lambda\right) & =\frac{\left(e\left(\left(-\hat{\gamma}_{3}+\omega \pm i \lambda\right) / \omega_{2}\right) ; q\right)_{\infty}}{\left(e\left(\left(\hat{\gamma}_{2}+\omega \pm i \lambda\right) / \omega_{2}\right) ; q\right)_{\infty}} \phi(\gamma ; x, \lambda), \\
\phi\left(\gamma_{0}, \gamma_{2}, \gamma_{1}, \gamma_{3} ; x, \lambda\right) & =\phi(\gamma ; x, \lambda), \\
\phi\left(\gamma_{0}, \gamma_{1},-\gamma_{3},-\gamma_{2} ; x, \lambda\right) & =\frac{\left(e\left(\left(-\gamma_{3}+\omega \pm i x\right) / \omega_{2}\right) ; q\right)_{\infty}}{\left(e\left(\left(\gamma_{2}+\omega \pm i x\right) / \omega_{2}\right) ; q\right)_{\infty}} \phi(\gamma ; x, \lambda) .
\end{aligned}
$$

Proof. Similarly as for the $R$-function (see Proposition 4.18), the symmetries of the Askey-Wilson function correspond to the $W\left(D_{5}\right)$-symmetries of $B_{t}$. Alternatively, all symmetries follow trivially from the series expansion (5.22) of the Askey-Wilson function, besides its symmetry with respect to $\gamma_{0} \leftrightarrow \gamma_{1}$ and $\gamma_{2} \leftrightarrow-\gamma_{3}$. These two symmetry relations are equivalent under duality, since

$$
\left(\gamma_{1}, \gamma_{0}, \gamma_{2}, \gamma_{3}\right)=\left(\hat{\gamma}_{0}, \hat{\gamma}_{1},-\hat{\gamma}_{3},-\hat{\gamma}_{2}\right),
$$

so we only discuss the symmetry with respect to $\gamma_{0} \leftrightarrow \gamma_{1}$. By Proposition 5.20 we have, with parameters $t$ given by (5.20) and (5.18),

$$
\begin{aligned}
\phi\left(\gamma_{1}, \gamma_{0}, \gamma_{2}, \gamma_{3} ; x, \lambda\right) & =\frac{\left(q, e\left(\left(\gamma_{1}+\omega \pm i x\right) / \omega_{2}\right), e\left(\left(\hat{\gamma}_{0}+\omega \pm i \lambda\right) / \omega_{2}\right) ; q\right)_{\infty}}{2} B_{t}\left(w s_{35} t\right) \\
& =\frac{\left(e\left(\left(-\hat{\gamma}_{3}+\omega \pm i \lambda\right) / \omega_{2}\right) ; q\right)_{\infty}}{\left(e\left(\left(\hat{\gamma}_{2}+\omega \pm i \lambda\right) / \omega_{2}\right) ; q\right)_{\infty}} \phi(\gamma ; x, \lambda),
\end{aligned}
$$

as desired.

Next we show that the Askey-Wilson function satisfies the same Askey-Wilson second order difference equation (with step-size $i \omega_{1}$ ) as Ruijsenaars' $R$-function, a result which has previously been derived from detailed studies of the associated Askey-Wilson polynomials in [9], cf. also [11].

Lemma 5.28. The Askey-Wilson function $\phi(\gamma ; x, \lambda)$ satisfies the second order difference equation

$$
\begin{aligned}
& A\left(\gamma ; x ; \omega_{1}, \omega_{2}\right)\left(\phi\left(\gamma ; x+i \omega_{1}, \lambda ; \omega_{1}, \omega_{2}\right)-\phi\left(\gamma ; x, \lambda ; \omega_{1}, \omega_{2}\right)\right)+(x \leftrightarrow-x) \\
& \quad=B\left(\gamma ; \lambda ; \omega_{1}, \omega_{2}\right) \phi\left(\gamma ; x, \lambda ; \omega_{1}, \omega_{2}\right),
\end{aligned}
$$

where $A$ and $B$ are given by

$$
\begin{aligned}
& A\left(\gamma ; x ; \omega_{1}, \omega_{2}\right)=\frac{\prod_{j=0}^{3} \sinh \left(\pi\left(i \omega+x+i \gamma_{j}\right) / \omega_{2}\right)}{\sinh \left(2 \pi x / \omega_{2}\right) \sinh \left(2 \pi(i \omega+x) / \omega_{2}\right)}, \\
& B\left(\gamma ; \lambda ; \omega_{1}, \omega_{2}\right)=\sinh \left(\pi\left(\lambda-i \omega-i \hat{\gamma}_{0}\right) / \omega_{2}\right) \sinh \left(\pi\left(\lambda+i \omega+i \hat{\gamma}_{0}\right) / \omega_{2}\right) .
\end{aligned}
$$

Proof. Specialize the parameters according to (5.20) and (5.18) in (5.17). Subsequently express $B_{t}(t), B_{t}\left(\tau_{45} t\right)$ and $B_{t}\left(\tau_{54} t\right)$ in terms of $\phi(\gamma ; x, \lambda), \phi\left(\gamma ; x+i \omega_{1}, \lambda\right)$ and $\phi(\gamma ; x-$ $\left.i \omega_{1}, \lambda\right)$ respectively. The resulting equation is the desired difference equation. 
Remark 5.29. Denoting $\Phi(z ; \mu)=\Phi(a, b, c, d ; z, \mu)$ for the Askey-Wilson function in the usual Askey-Wilson parameters, Lemma 5.28 becomes the Askey-Wilson second order difference equation

$$
\begin{aligned}
& A(z)(\Phi(q z, \mu)-\Phi(z, \mu))+A\left(z^{-1}\right)(\Phi(z / q, \mu)-\Phi(z, \mu)) \\
& \quad=\left(\widetilde{a}\left(\gamma+\gamma^{-1}\right)-1-\widetilde{a}^{2}\right) \Phi(z, \mu),
\end{aligned}
$$

where

$$
A(z)=\frac{(1-a z)(1-b z)(1-c z)(1-d z)}{\left(1-q z^{2}\right)\left(1-z^{2}\right)}
$$

and $\widetilde{a}=e\left(\left(\hat{\gamma}_{0}+\omega\right) / \omega_{2}\right)$.

We have now seen that the $R$-function $R\left(\gamma ; x, \lambda ; \omega_{1}, \omega_{2}\right)$ as well as the Askey-Wilson function $\phi\left(\gamma ; x, \lambda ; \omega_{1}, \omega_{2}\right)$ are solutions to the eigenvalue problem

$$
\mathcal{L}_{\gamma}^{\omega_{1}, \omega_{2}} f=B\left(\gamma ; \lambda ; \omega_{1}, \omega_{2}\right) f
$$

for the Askey-Wilson second order difference operator $\mathcal{L}_{\gamma}^{\omega_{1}, \omega_{2}}$ (4.31) with step-size $i \omega_{1}$. These two solutions have essentially different behaviour in the $i \omega_{2}$-step direction: the Askey-Wilson function $\phi(\gamma ; x, \lambda)$ is $i \omega_{2}$-periodic, while the $R$-function $R(\gamma ; x, \lambda)$ is $\omega_{1} \leftrightarrow \omega_{2}$ invariant (hence is also an eigenfunction of the Askey-Wilson second order difference operator $\mathcal{L}_{\gamma}^{\omega_{2}, \omega_{1}}$ with step-size $i \omega_{2}$, with eigenvalue $\left.B\left(\gamma ; \lambda ; \omega_{2}, \omega_{1}\right)\right)$. On the other hand, note that $\widetilde{\tau}=-\omega_{2} / \omega_{1} \in \mathbb{H}_{+}$and that

$A\left(\gamma ; x ; \omega_{2}, \omega_{1}\right)=A\left(-\gamma ;-x ;-\omega_{2}, \omega_{1}\right), \quad B\left(\gamma ; \lambda ; \omega_{2}, \omega_{1}\right)=B\left(-\gamma ; \lambda ;-\omega_{2}, \omega_{1}\right)$

with $-\gamma=\left(-\gamma_{0},-\gamma_{1},-\gamma_{2},-\gamma_{3}\right)$, so that the Askey-Wilson function $\phi\left(-\gamma ; x, \lambda ;-\omega_{2}\right.$, $\omega_{1}$ ) (with associated modular inverted base $\tilde{q}=e\left(-\omega_{2} / \omega_{1}\right)$ ) does satisfy the AskeyWilson second order difference equation

$$
\left(\mathcal{L}_{\gamma}^{\omega_{2}, \omega_{1}} \phi\left(-\gamma ; \cdot, \lambda ;-\omega_{2}, \omega_{1}\right)\right)(x)=B\left(\gamma ; \lambda ; \omega_{2}, \omega_{1}\right) \phi\left(-\gamma ; x, \lambda ;-\omega_{2}, \omega_{1}\right),
$$

cf. $[27, \S 6.6]$. In the next section we match the hyperbolic theory to the trigonometric theory, which in particular entails an explicit expression of the $R$-function in terms of products of Askey-Wilson functions in base $q$ and base $\widetilde{q}$.

Note furthermore that Proposition 5.27 hints at the fact that the solution space to the Askey-Wilson eigenvalue problem (5.23) admits a natural twisted $W\left(D_{4}\right)$-action on the parameters $\gamma$. In fact, the solution space to (5.23) is invariant under permutations of $\left(\gamma_{0}, \gamma_{1}, \gamma_{2}, \gamma_{3}\right)$. Furthermore, a straightforward computation shows that

$$
\begin{aligned}
g(\gamma ; \cdot)^{-1} \circ \mathcal{L}_{\gamma}^{\omega_{1}, \omega_{2}} \circ g(\gamma ; \cdot)= & \mathcal{L}_{\left(\gamma_{0}, \gamma_{1},-\gamma_{3},-\gamma_{2}\right)}^{\omega_{1}}+B\left(\gamma ; \lambda ; \omega_{1}, \omega_{2}\right) \\
& -B\left(\gamma_{0}, \gamma_{1},-\gamma_{3},-\gamma_{2} ; \lambda ; \omega_{1}, \omega_{2}\right)
\end{aligned}
$$

for the gauge factor

$$
g(\gamma ; x)=\frac{\left(e\left(\left(\gamma_{2}+\omega \pm i x\right) / \omega_{2}\right) ; q\right)_{\infty}}{\left(e\left(\left(-\gamma_{3}+\omega \pm i x\right) / \omega_{2}\right) ; q\right)_{\infty}},
$$

which implies that for a given solution $F_{\lambda}\left(\gamma_{0}, \gamma_{1},-\gamma_{3},-\gamma_{2} ; \cdot\right)$ to the eigenvalue problem

$$
\mathcal{L}_{\left(\gamma_{0}, \gamma_{1},-\gamma_{3},-\gamma_{2}\right)}^{\omega_{1}, \omega_{2}} f=B\left(\gamma_{0}, \gamma_{1},-\gamma_{3},-\gamma_{2} ; \lambda ; \omega_{1}, \omega_{2}\right) f
$$


we obtain a solution

$$
\widetilde{F}_{\lambda}(\gamma ; x):=g(\gamma ; x) F_{\lambda}\left(\gamma_{0}, \gamma_{1},-\gamma_{3},-\gamma_{2} ; x\right)
$$

to the eigenvalue problem (5.23). A similar observation forms the starting point of Ruijsenaars' [28] analysis of the $W\left(D_{4}\right)$-symmetries of the $R$-function (see also Sect. 4.6).

Remark 5.30. A convenient way to formalize the $W\left(D_{4}\right)$-symmetries of the eigenvalue problem (5.23) (in the present trigonometric setting) is by interpreting $i \omega_{2}$-periodic solutions to (5.23), depending meromorphically on $(\gamma, x, \lambda)$, as defining a sub-vector bundle $\widetilde{\Gamma}^{0}\left(\omega_{1}, \omega_{2}\right)$ of the meromorphic vector bundle $\Gamma^{0}\left(\omega_{1}, \omega_{2}\right)$ over

$$
X=\left(\mathbb{C} / \mathbb{Z} \omega_{2}\right)^{4} \times \mathbb{C} /\left(\mathbb{Z} i \omega_{1}+\mathbb{Z} i \omega_{2}\right) \times \mathbb{C} / \mathbb{Z} i \omega_{2}
$$

consisting of meromorphic functions in $(\gamma, x, \lambda) \in\left(\mathbb{C} / \mathbb{Z} \omega_{2}\right)^{4} \times \mathbb{C} / \mathbb{Z} i \omega_{2} \times \mathbb{C} / \mathbb{Z} i \omega_{2}$. The above analysis can now equivalently be reformulated as the following property of $\widetilde{\Gamma}^{0}\left(\omega_{1}, \omega_{2}\right)$ : the sub-vector bundle $\widetilde{\Gamma}^{0}\left(\omega_{1}, \omega_{2}\right)$ is $W\left(D_{4}\right)$-invariant with respect to the twisted $W\left(D_{4}\right)$-action

$$
(\sigma \cdot f)(\gamma ; x, \lambda):=V_{\sigma}(\gamma ; x, \lambda)^{-1} f\left(\sigma^{-1} \gamma ; x, \lambda\right), \quad \sigma \in W\left(D_{4}\right)
$$

on $\Gamma^{0}\left(\omega_{1}, \omega_{2}\right)$, where $V_{\sigma}(\gamma ; x, \lambda)=h\left(\sigma^{-1} \gamma ; x, \lambda\right) / h(\gamma ; x, \lambda)\left(\sigma \in W\left(D_{4}\right)\right)$ is the 1 -coboundary with $h(\gamma ; x, \lambda)=h\left(\gamma ; x, \lambda ; \omega_{1}, \omega_{2}\right)$ e.g. given by

$$
\begin{aligned}
& h\left(\gamma ; x, \lambda ; \omega_{1}, \omega_{2}\right) \\
& \quad=\frac{\theta\left(e\left(\left(\gamma_{3}-\hat{\gamma}_{0}+i x\right) / \omega_{2}\right) ; q\right)}{\theta\left(e\left(\left(\omega-\gamma_{3}-i x\right) / \omega_{2}\right) ; q\right) \prod_{j=0}^{3}\left(e\left(\left(\omega-\gamma_{j}+i x\right) / \omega_{2}\right) ; q\right)_{\infty}} \in \Gamma_{0}\left(\omega_{1}, \omega_{2}\right)^{\times},
\end{aligned}
$$

and where $W\left(D_{4}\right)$ acts on the $\gamma$ parameters by permutations and even sign changes. By a straightforward analysis using Casorati-determinants and the asymptotically free solutions to the eigenvalue problem $(5.23)$, one can furthermore show that $\widetilde{\Gamma}^{0}\left(\omega_{1}, \omega_{2}\right)$ is a (trivial) meromorphic vector bundle over $X$ of rank two (compare with the general theory on difference equations in [19]).

We end this subsection by expressing the Askey-Wilson function $\phi(\gamma ; x, \lambda)$ in terms of the trigonometric integrals $E_{t}$ and $V_{t}$ using Proposition 5.23. Note its close resemblance with the hyperbolic case, cf. Theorem 4.21.

Lemma 5.31. a) We have

$$
\begin{aligned}
& \phi(\gamma ; x, \lambda) \\
& =\frac{(q ; q)_{\infty}}{2} \frac{\left(e\left(\left(\hat{\gamma}_{0}+\omega-i \lambda\right) / \omega_{2}\right), e\left(\left(\hat{\gamma}_{1}+\omega+i \lambda\right) / \omega_{2}\right), e\left(\left(\hat{\gamma}_{2}+\omega+i \lambda\right) / \omega_{2}\right) ; q\right)_{\infty}}{\left(e\left(\left(-\hat{\gamma}_{3}+\omega-i \lambda\right) / \omega_{2}\right) ; q\right)_{\infty}} \\
& \quad \times \frac{\prod_{j=0}^{2}\left(e\left(\left(\gamma_{j}+\omega \pm i x\right) / \omega_{2}\right) ; q\right)_{\infty}}{\left(e\left(\left(-\gamma_{3}+\omega \pm i x\right) / \omega_{2}\right) ; q\right)_{\infty}} E_{t}(t)
\end{aligned}
$$

with

$$
\begin{aligned}
t_{1} & =e\left(\left(-\frac{3 \omega}{2}+\gamma_{3}-\frac{\hat{\gamma}_{0}}{2}+\frac{i \lambda}{2}\right) / \omega_{2}\right), & t_{2} & =e\left(\left(\frac{\omega}{2}+\gamma_{2}-\frac{\hat{\gamma}_{0}}{2}+\frac{i \lambda}{2}\right) / \omega_{2}\right), \\
t_{3} & =e\left(\left(\frac{\omega}{2}+\gamma_{1}-\frac{\hat{\gamma}_{0}}{2}+\frac{i \lambda}{2}\right) / \omega_{2}\right), & t_{4} & =e\left(\left(\frac{\omega}{2}+\gamma_{0}-\frac{\hat{\gamma}_{0}}{2}+\frac{i \lambda}{2}\right) / \omega_{2}\right), \\
t_{5} & =e\left(\left(\frac{\omega}{2}+\frac{\hat{\gamma}_{0}}{2}+i x-\frac{i \lambda}{2}\right) / \omega_{2}\right), & t_{6} & =e\left(\left(\frac{\omega}{2}+\frac{\hat{\gamma}_{0}}{2}-i x-\frac{i \lambda}{2}\right) / \omega_{2}\right) .
\end{aligned}
$$


b) We have

$$
\begin{aligned}
& \phi(\gamma ; x, \lambda) \\
& =\frac{(q ; q)_{\infty}}{2} \frac{\left(e\left(\left(\hat{\gamma}_{0}+\omega+i \lambda\right) / \omega_{2}\right), e\left(\left(\hat{\gamma}_{1}+\omega-i \lambda\right) / \omega_{2}\right), e\left(\left(\hat{\gamma}_{2}+\omega-i \lambda\right) / \omega_{2}\right) ; q\right)_{\infty}}{\left(e\left(\left(-\hat{\gamma}_{3}+\omega+i \lambda\right) / \omega_{2}\right) ; q\right)_{\infty}} V_{t}(t)
\end{aligned}
$$

with

$$
\begin{array}{ll}
t_{1}=e\left(\left(\frac{3 \omega}{2}-\gamma_{3}+\frac{\hat{\gamma}_{0}}{2}-\frac{i \lambda}{2}\right) / \omega_{2}\right), & t_{2}=e\left(\left(-\frac{\omega}{2}-\gamma_{2}+\frac{\hat{\gamma}_{0}}{2}-\frac{i \lambda}{2}\right) / \omega_{2}\right), \\
t_{3}=e\left(\left(-\frac{\omega}{2}-\gamma_{1}+\frac{\hat{\gamma}_{0}}{2}-\frac{i \lambda}{2}\right) / \omega_{2}\right), & t_{4}=e\left(\left(-\frac{\omega}{2}-\gamma_{0}+\frac{\hat{\gamma}_{0}}{2}-\frac{i \lambda}{2}\right) / \omega_{2}\right), \\
t_{5}=e\left(\left(-\frac{\omega}{2}-\frac{\hat{\gamma}_{0}}{2}+i x+\frac{i \lambda}{2}\right) / \omega_{2}\right), & t_{6}=e\left(\left(-\frac{\omega}{2}-\frac{\hat{\gamma}_{0}}{2}-i x+\frac{i \lambda}{2}\right) / \omega_{2}\right) .
\end{array}
$$

Proof. a) We use Proposition 5.27 to rewrite $\phi(\gamma ; x, \lambda)$ in terms of $\phi\left(\gamma_{0}, \gamma_{1},-\gamma_{3},-\gamma_{2}\right.$;

$x,-\lambda)$. Subsequently we use the defining expression of $\phi\left(\gamma_{0}, \gamma_{1},-\gamma_{3},-\gamma_{2} ; x,-\lambda\right)$ to obtain

$$
\begin{aligned}
& \phi(\gamma ; x, \lambda) \\
& =\frac{\left(q, e\left(\left(\hat{\gamma}_{1}+\omega \pm i \lambda\right) / \omega_{2}\right), e\left(\left(\gamma_{0}+\omega \pm i x\right) / \omega_{2}\right), e\left(\left(\gamma_{2}+\omega \pm i x\right) / \omega_{2}\right) ; q\right)_{\infty}}{2\left(e\left(\left(-\gamma_{3}+\omega \pm i x\right) / \omega_{2}\right) ; q\right)_{\infty}} B_{t}(\xi)
\end{aligned}
$$

with

$$
\begin{aligned}
\xi= & \left(e\left(\left(-\gamma_{0}-\gamma_{1}-2 \omega\right) / \omega_{2}\right), 1, e\left(\left(\hat{\gamma}_{1}+\omega+i \lambda\right) / \omega_{2}\right), e\left(\left(\gamma_{0}+\omega-i x\right) / \omega_{2}\right),\right. \\
& e\left(\left(\gamma_{0}+\omega+i x\right) / \omega_{2}\right), e\left(\left(\hat{\gamma}_{1}+\omega-i \lambda\right) / \omega_{2}\right), e\left(\left(\gamma_{2}-\gamma_{0}\right) / \omega_{2}\right), \\
& \left.e\left(\left(\gamma_{3}-\gamma_{0}-2 \omega\right) / \omega_{2}\right)\right) .
\end{aligned}
$$

With this specific ordered set $\xi$ of parameters we apply Proposition 5.23 to rewrite $B_{t}(\xi)$ in terms of $E_{t}$, which results in the desired identity.

b) This follows from applying Proposition 5.23 directly to the definition (5.21) of $\phi(\gamma ; x, \lambda)$.

Using the expression of the Askey-Wilson function in terms of $V_{t}$ and using Lemma 5.17, we thus obtain an expression of the Askey-Wilson function as very-wellpoised ${ }_{8} \phi_{7}$ series.

\section{Hyperbolic Versus Trigonometric Theory}

6.1. Hyperbolic versus trigonometric gamma functions. We fix throughout this section periods $\omega_{1}, \omega_{2} \in \mathbb{C}$ with $\Re\left(\omega_{1}\right)>0, \Re\left(\omega_{2}\right)>0$ and $\tau=\omega_{1} / \omega_{2} \in \mathbb{H}_{+}$. We set

$$
q=q_{\omega_{1}, \omega_{2}}=e\left(\omega_{1} / \omega_{2}\right), \quad \widetilde{q}=\widetilde{q}_{\omega_{1}, \omega_{2}}=e\left(-\omega_{2} / \omega_{1}\right),
$$

where $e(x)=\exp (2 \pi i x)$ as before, so that $|q|,|\widetilde{q}|<1$.

Shintani's [30] product expansion is

$$
G\left(\omega_{1}, \omega_{2} ; x\right)=e\left(-\frac{1}{48}\left(\frac{\omega_{1}}{\omega_{2}}+\frac{\omega_{2}}{\omega_{1}}\right)\right) e\left(-\frac{x^{2}}{4 \omega_{1} \omega_{2}}\right) \frac{\left(e\left((i x+\omega) / \omega_{2}\right) ; q\right)_{\infty}}{\left(e\left((i x-\omega) / \omega_{1}\right) ; \widetilde{q}\right)_{\infty}}
$$


where $\omega=\frac{1}{2}\left(\omega_{1}+\omega_{2}\right)$ as before. For a proof of (6.1), see [37, Prop. A.1]. In other words, the product expansion (6.1) expresses the hyperbolic gamma function as a quotient of two trigonometric gamma functions (one in base $q$, the other in the modular inverted base $\widetilde{q})$. In this section we explicitly write the base-dependence; e.g. we write $S_{t}(t ; q)$ $\left(t \in \mathcal{H}_{1}\right)$ to denote the trigonometric hypergeometric function $S_{t}(t)$ in base $q$.

6.2. Hyperbolic versus trigonometric hypergeometric integrals. We explore (6.1) to relate the hyperbolic integrals to their trigonometric analogs. We start with the hyperbolic hypergeometric function $S_{h}(u)\left(u \in \mathcal{G}_{2 i \omega}\right)$. For $u \in \mathcal{G}_{2 i \omega}$ we write

$$
t_{j}=e\left(\left(i u_{j}+\omega\right) / \omega_{2}\right), \quad \widetilde{t}_{j}=e\left(\left(i u_{j}-\omega\right) / \omega_{1}\right), \quad j=1, \ldots, 8 .
$$

Observe that $\prod_{j=1}^{8} t_{j}=q^{2}$ and $\prod_{j=1}^{8} \widetilde{t}_{j}=\widetilde{q}^{6}$.

Theorem 6.1. As meromorphic functions of $u \in \mathcal{G}_{2 i \omega}$ we have

$$
\begin{aligned}
S_{h}(u)= & \omega_{2} e\left(\left(2 \omega^{2}+\sum_{j=1}^{5} u_{j}^{2}-u_{6}^{2}+u_{7}^{2}-u_{8}^{2}\right) / 2 \omega_{1} \omega_{2}\right) \frac{(\widetilde{q}, \widetilde{q} ; \widetilde{q})_{\infty}}{2} \frac{\prod_{j=1}^{5} \theta\left(\widetilde{t}_{j} \widetilde{t}_{7} / \widetilde{q} ; \widetilde{q}\right)}{\theta\left(\widetilde{t}_{7} / \widetilde{t}_{6} ; \widetilde{q}\right)} \\
& \times U_{t}\left(\widetilde{q}^{\frac{3}{2}} / \widetilde{t}_{8}, \widetilde{q}^{\frac{1}{2}} / \widetilde{t}_{1}, \ldots, \widetilde{q}^{\frac{1}{2}} / \widetilde{t}_{6}, \widetilde{q}^{\frac{3}{2}} / \widetilde{t_{7}} ; \widetilde{q}\right) S_{t}\left(t_{6} / q, t_{1}, \ldots, t_{5}, t_{7}, t_{8} / q ; q\right) \\
& +\left(u_{6} \leftrightarrow u_{7}\right),
\end{aligned}
$$

with the parameters $t_{j}$ and $\widetilde{t}_{j}$ given by (6.2).

Proof. We put several additional conditions on the parameters, which can later be removed by analytic continuity. We assume that $\omega_{1},-\omega_{2} \in \mathbb{H}_{+}$and that $\Re(i \omega)<0$. We furthermore choose parameters $u \in \mathcal{G}_{2 i \omega}$ satisfying $\Re\left(u_{j}-i \omega\right)>0$ and $\Im\left(u_{j}-i \omega\right)<0$ for $j=1, \ldots, 8$. Then

$$
\begin{aligned}
S_{h}(u) & =\int_{\mathbb{R}} \frac{G(i \omega \pm 2 x)}{\prod_{j=1}^{8} G\left(u_{j} \pm x\right)} d x \\
& =e\left(\frac{7}{24}\left(\frac{\omega_{1}}{\omega_{2}}+\frac{\omega_{2}}{\omega_{1}}\right)\right) e\left(\left(\omega^{2}+\sum_{j=1}^{8} u_{j}^{2}\right) / 2 \omega_{1} \omega_{2}\right) \int_{\mathbb{R}} W(x) \widetilde{W}(x) d x,
\end{aligned}
$$

where

$$
\begin{aligned}
& W(x)=\frac{\left(e\left( \pm 2 i x / \omega_{2}\right) ; q\right)_{\infty}}{\prod_{j=1}^{8}\left(t_{j} e\left( \pm i x / \omega_{2}\right) ; q\right)_{\infty}} \\
& \widetilde{W}(x)=e\left(2 x^{2} / \omega_{1} \omega_{2}\right) \frac{\prod_{j=1}^{8}\left(\widetilde{t}_{j} e\left( \pm i x / \omega_{1}\right) ; \widetilde{q}\right)_{\infty}}{\left(\widetilde{q} e\left( \pm 2 i x / \omega_{1}\right) ; \widetilde{q}\right)_{\infty}}
\end{aligned}
$$

by (6.1). Using Cauchy's Theorem and elementary asymptotic estimates of the integrand, we may rotate the integration contour $\mathbb{R}$ to $i \omega_{2} \mathbb{R}$. Since the factor $W(x)$ is $i \omega_{2}$-periodic, we can fold the resulting integral, interchange summation and integration by Fubini's Theorem, to obtain the expression

$$
S_{h}(u)=e\left(\frac{7}{24}\left(\frac{\omega_{1}}{\omega_{2}}+\frac{\omega_{2}}{\omega_{1}}\right)\right) e\left(\left(\omega^{2}+\sum_{j=1}^{8} u_{j}^{2}\right) / 2 \omega_{1} \omega_{2}\right) \int_{0}^{i \omega_{2}} W(x) F(x) d x,
$$


where

$$
\begin{aligned}
F(x) & =\sum_{n=-\infty}^{\infty} \widetilde{W}\left(x+m i \omega_{2}\right) \\
& =\widetilde{W}(x)_{10} \psi_{10}\left(\begin{array}{c}
\widetilde{q} e\left(i x / \omega_{1}\right),-\widetilde{q} e\left(i x / \omega_{1}\right),\left\{\widetilde{q}_{j}^{-1} e\left(i x / \omega_{1}\right)\right\}_{j=1}^{8} ; \widetilde{q}, \widetilde{q} \\
e\left(i x / \omega_{1}\right),-e\left(i x / \omega_{1}\right),\left\{\widetilde{t}_{j} e\left(i x / \omega_{1}\right)\right\}_{j=1}^{8}
\end{array}\right) .
\end{aligned}
$$

At this stage we have to resort to [5, (5.6.3)], which expresses a very-well-poised ${ }_{10} \psi_{10}$ bilateral series as a sum of three very-well-poised ${ }_{10} \phi_{9}$ unilateral series. This results in the formula

$$
\begin{aligned}
F(x)= & e\left(2 x^{2} / \omega_{1} \omega_{2}\right) \theta\left(\widetilde{t}_{6} e\left( \pm i x / \omega_{1}\right), \widetilde{t}_{7} e\left( \pm i x / \omega_{1}\right) ; \widetilde{q}\right) \\
& \times \frac{(\widetilde{q} ; \widetilde{q})_{\infty} \prod_{j=1}^{5}\left(\widetilde{t}_{j} \widetilde{t}_{8} / \widetilde{q}, \widetilde{q} \widetilde{t}_{j} / \widetilde{t}_{8} ; \widetilde{q}\right)_{\infty}}{\left(\widetilde{q}^{2} / \widetilde{t}_{6} \widetilde{t}_{8}, \widetilde{q}^{2} / \widetilde{t}_{7} \widetilde{t}_{8}, \widetilde{t}_{8} / \widetilde{t}_{6}, \widetilde{t}_{8} / \widetilde{t}_{7}, \widetilde{q}^{3} / \widetilde{t}_{8}^{2} ; \widetilde{q}\right)_{\infty}} 10 W_{9}\left(\widetilde{q}^{2} / \widetilde{t}_{8}^{2} ;\left\{\widetilde{q}^{2} / \widetilde{t}_{j} \widetilde{t}_{8}\right\}_{j=1}^{7} ; \widetilde{q}, \widetilde{q}\right) \\
& +\left(u_{8} ; u_{6}, u_{7}\right),
\end{aligned}
$$

where $\left(u_{8} ; u_{6}, u_{7}\right)$ means cyclic permutation of the parameters $\left(u_{8}, u_{6}, u_{7}\right)$. Note that the ${ }_{10} \phi_{9}$ series in the expression of $F(x)$ are independent of $x$. Combining Jacobi's inversion formula, the Jacobi triple product identity and the modularity

$$
\frac{(q ; q)_{\infty}}{(\widetilde{q} ; \widetilde{q})_{\infty}}=\sqrt{\frac{\omega_{2}}{-i \omega_{1}}} e\left(-\frac{1}{24}\left(\frac{\omega_{1}}{\omega_{2}}+\frac{\omega_{2}}{\omega_{1}}\right)\right)
$$

of Dedekind's eta function, we obtain

$$
\theta\left(e\left(u / \omega_{1}\right) ; \widetilde{q}\right)=e\left(-\frac{1}{24}\left(\frac{\omega_{1}}{\omega_{2}}+\frac{\omega_{2}}{\omega_{1}}\right)\right) e\left((u+\omega)^{2} / 2 \omega_{1} \omega_{2}\right) \theta\left(e\left(-u / \omega_{2}\right) ; q\right)
$$

for the rescaled Jacobi theta function $\theta(\cdot)$, see e.g. [5] or [37]. As a result, we can rewrite the theta functions in the expression of $F(x)$ as theta functions in base $q$,

$$
\begin{aligned}
& e\left(2 x^{2} / \omega_{1} \omega_{2}\right) \theta\left(\tilde{t}_{6} e\left( \pm i x / \omega_{1}\right), \tilde{t}_{7} e\left( \pm i x / \omega_{1}\right) ; \widetilde{q}\right) \\
& \quad=e\left(-\frac{1}{6}\left(\frac{\omega_{1}}{\omega_{2}}+\frac{\omega_{2}}{\omega_{1}}\right)\right) e\left(-\left(u_{6}^{2}+u_{7}^{2}\right) / \omega_{1} \omega_{2}\right) \theta\left(q e\left( \pm i x / \omega_{2}\right) / t_{6}, q e\left( \pm i x / \omega_{2}\right) / t_{7} ; q\right) .
\end{aligned}
$$

We thus obtain the expression

$$
\begin{aligned}
S_{h}(u)= & C\left(u_{8} ; u_{6}, u_{7}\right) \int_{0}^{i \omega_{2}} \theta\left(q e\left( \pm i x / \omega_{2}\right) / t_{6}, q e\left( \pm i x / \omega_{2}\right) / t_{7} ; q\right) W(x) d x \\
& +\left(u_{8} ; u_{6}, u_{7}\right) \\
= & i \omega_{2} C\left(u_{8} ; u_{6}, u_{7}\right) S_{t}\left(t_{6} / q, t_{1}, \ldots, t_{5}, t_{8}, t_{7} / q ; q\right)+\left(u_{8} ; u_{6}, u_{7}\right),
\end{aligned}
$$

where we have used that $\left|t_{j}\right|<1$ for $j=1, \ldots, 6$, with

$$
\begin{aligned}
C\left(u_{8} ; u_{6}, u_{7}\right)= & e\left(\frac{1}{8}\left(\frac{\omega_{1}}{\omega_{2}}+\frac{\omega_{2}}{\omega_{1}}\right)\right) e\left(\left(\omega^{2}+\sum_{j=1}^{5} u_{j}^{2}-u_{6}^{2}-u_{7}^{2}+u_{8}^{2}\right) / 2 \omega_{1} \omega_{2}\right) \\
& \times \frac{(\widetilde{q} ; \widetilde{q})_{\infty} \prod_{j=1}^{5}\left(\widetilde{t_{j}} \widetilde{t_{8}} / \widetilde{q}, \widetilde{q} \widetilde{t}_{j} / \widetilde{t}_{8} ; \widetilde{q}\right)_{\infty}}{\left(\widetilde{q}^{2} / \widetilde{t}_{6} \widetilde{t}_{8}, \widetilde{q}^{2} / \widetilde{t}_{7} \widetilde{t}_{8}, \widetilde{t}_{8} / \widetilde{t}_{6}, \widetilde{t}_{8} / \widetilde{t}_{7}, \widetilde{q}^{3} / \widetilde{t}_{8}^{2} ; \widetilde{q}\right)_{\infty}} \\
& \times{ }_{10} W_{9}\left(\widetilde{q}^{2} / \widetilde{t}_{8}^{2} ;\left\{\widetilde{q}^{2} / \widetilde{t}_{j} \widetilde{t}_{8}\right\}_{j=1}^{7} ; \widetilde{q}, \widetilde{q}\right) .
\end{aligned}
$$


We thus have obtained an expression of $S_{h}(u)$ as a sum of three trigonometric hypergeometric functions $S_{t}$ in base $q$, with coefficients expressed as very-well-poised ${ }_{10} \phi_{9}$ series in base $\tilde{q}$. The next step is to use a three term transformation for $S_{t}$ to write $S_{h}(u)$ as a sum of two trigonometric hypergeometric functions $S_{t}$ in base $q$, with coefficients now being a sum of two very-well-poised ${ }_{10} \phi_{9}$ series.

Concretely, we consider the contiguous relation (3.8) for $S_{e}$ with $p \leftrightarrow q$ and with parameters specialized to $\left(t_{1}, \ldots, t_{5}, p t_{6}, p t_{7}, t_{8}\right)$. Taking the limit $p \rightarrow 0$ leads to the three term transformation

$$
\begin{aligned}
S_{t}\left(t_{6} / q, t_{1}, \ldots, t_{5}, t_{8}, t_{7} / q ; q\right)= & \frac{\theta\left(t_{7} / t_{8} ; q\right)}{\theta\left(t_{7} / t_{6} ; q\right)} \prod_{j=1}^{5} \frac{\theta\left(t_{j} t_{6} ; q\right)}{\theta\left(t_{j} t_{8} ; q\right)} \\
& \times S_{t}\left(t_{7} / q, t_{1}, \ldots, t_{6}, t_{8} / q ; q\right)+\left(u_{6} \leftrightarrow u_{7}\right)(6
\end{aligned}
$$

for $S_{t}$. Rewriting the coefficients in (6.7) in base $\widetilde{q}$ using the Jacobi inversion formula $(6.5)$

$$
\frac{\theta\left(t_{7} / t_{8} ; q\right)}{\theta\left(t_{7} / t_{6} ; q\right)} \prod_{j=1}^{5} \frac{\theta\left(t_{j} t_{6} ; q\right)}{\theta\left(t_{j} t_{8} ; q\right)}=e\left(\left(u_{6}^{2}-u_{8}^{2}\right) / \omega_{1} \omega_{2}\right) \frac{\theta\left(\widetilde{t}_{8} / \widetilde{t}_{7} ; \widetilde{q}\right)}{\theta\left(\widetilde{t}_{6} / \widetilde{t}_{7} ; \widetilde{q}\right)} \prod_{j=1}^{5} \frac{\theta\left(\widetilde{q}^{2} / \widetilde{t}_{j} \widetilde{t}_{6} ; \widetilde{q}\right)}{\theta\left(\widetilde{q}^{2} / \widetilde{t}_{j} \widetilde{t}_{8} ; \widetilde{q}\right)},
$$

and using the resulting three term transformation in (6.6), we obtain

$$
S_{h}(u)=D\left(u_{6}, u_{7}\right) S_{t}\left(t_{7} / q, t_{1}, \ldots, t_{6}, t_{8} / q ; q\right)+\left(u_{6} \leftrightarrow u_{7}\right)
$$

with

$$
\begin{aligned}
D\left(u_{6}, u_{7}\right)= & i \omega_{2}\left(e\left(\left(u_{6}^{2}-u_{8}^{2}\right) / \omega_{1} \omega_{2}\right) \frac{\theta\left(\widetilde{t}_{8} / \widetilde{t}_{7} ; \widetilde{q}\right)}{\theta\left(\widetilde{t}_{6} / \widetilde{t}_{7} ; \widetilde{q}\right)}\right. \\
& \left.\times \prod_{j=1}^{5} \frac{\theta\left(\widetilde{q}^{2} / \widetilde{t_{j}} \widetilde{t}_{6} ; \widetilde{q}\right)}{\theta\left(\widetilde{q}^{2} / \widetilde{t}_{j} \widetilde{t}_{8} ; \widetilde{q}\right)} C\left(u_{8} ; u_{6}, u_{7}\right)+C\left(u_{6} ; u_{7}, u_{8}\right)\right) .
\end{aligned}
$$

The coefficient $D\left(u_{6}, u_{7}\right)$ is a sum of two very-well-poised ${ }_{10} \phi_{9}$ series in base $\widetilde{q}$, which can be expressed in terms of the trigonometric integral $U_{t}$ (in base $\widetilde{q}$ ) by direct computations using Lemma 5.5. This yields the desired result.

Remark 6.2. i) Note that the $W\left(E_{6}\right)$-symmetry of the trigonometric integrals $S_{t}$ and $U_{t}$ is upgraded to a $W\left(E_{7}\right)$-symmetry in Theorem 6.1 since the second term in the right-hand side of (6.3) is the first term with the role of $u_{6}$ and $u_{7}$ interchanged.

ii) Specializing the parameters in Theorem 6.1 to $u \in \mathcal{G}_{2 i \omega}$ with $u_{1}=-u_{6}$ (so that $t_{1} t_{6}=q$ and $\widetilde{t}_{1} \widetilde{t}_{6}=\widetilde{q}$ ), the left-hand side of the identity can be evaluated by the hyperbolic Nassrallah-Rahman integral evaluation (4.6). For the right-hand side of the identity, the second term vanishes because $\theta\left(\widetilde{t}_{1} \widetilde{t}_{6} / \widetilde{q} ; \widetilde{q}\right)=0$ under the particular parameter specialization. The remaining product of two trigonometric integrals can be evaluated by Corollary 5.9. The equality of both sides of the resulting identity can be reconfirmed using (6.1) and (6.4). It follows from this argument that the evaluation of the hyperbolic Nassrallah-Rahman integral is in fact a consequence of fusing trigonometric identities, an approach to hyperbolic beta integrals which was analyzed in detail in [37]. 
iii) More generally, specializing (6.3) at generic $u \in \mathcal{G}_{2 i \omega}$ satisfying $u_{1}+u_{6}=n i \omega_{1}+$ mi $\omega_{2}\left(n, m \in \mathbb{Z}_{\geq 0}\right.$ ), the second term on the right-hand side of (6.3) still vanishes while the first term reduces to the product of two terminating very-well-poised ${ }_{10} \phi_{9}$ series, one in base $q$ and the other in base $\widetilde{q}$. The terminating ${ }_{10} \phi_{9}$ series is Rahman's [20] biorthogonal rational ${ }_{10} \phi_{9}$ function (cf. Remark 5.13(ii)), while the resulting expression for $S_{h}$ is the corresponding two-index hyperbolic analogue of Rahman's biorthogonal rational function, considered by Spiridonov [33, §8.3] (cf. Remark 3.1 on the elliptic level).

Corollary 6.3. We have

$$
\begin{aligned}
E_{h}(u)= & \omega_{2} e\left(\frac{2 \omega^{2}+\sum_{j=1}^{4} u_{j}^{2}-u_{5}^{2}+u_{6}^{2}}{2 \omega_{1} \omega_{2}}\right) \frac{(\widetilde{q}, \widetilde{q} ; \widetilde{q})_{\infty}}{2} \frac{\prod_{j=1}^{4} \theta\left(\widetilde{t}_{j} \widetilde{t_{6}} / \widetilde{q} ; \widetilde{q}\right)}{\theta\left(\widetilde{t}_{6} / \widetilde{t}_{5} ; \widetilde{q}\right)} \\
& \times V_{t}\left(\frac{\widetilde{q}^{\frac{3}{2}}}{\widetilde{t}_{6}}, \frac{\widetilde{q}^{\frac{1}{2}}}{\widetilde{t}_{1}}, \ldots, \frac{\widetilde{q}^{\frac{1}{2}}}{\widetilde{t}_{5}} ; \widetilde{q}\right) E_{t}\left(\frac{t_{5}}{q}, t_{1}, \ldots, t_{4}, t_{6} ; q\right)+\left(u_{5} \leftrightarrow u_{6}\right)
\end{aligned}
$$

as meromorphic functions in $u \in \mathbb{C}^{6}$, where $t_{j}=e\left(\left(i u_{j}+\omega\right) / \omega_{2}\right)$ and $\tilde{t}_{j}=e\left(\left(i u_{j}-\right.\right.$ $\left.\omega) / \omega_{1}\right)(j=1, \ldots, 6)$ as before.

Proof. For generic $u \in \mathcal{G}_{2 i \omega}$ we have

$$
E_{h}\left(u_{2}, \ldots, u_{7}\right)=\lim _{s \rightarrow \infty} S_{h}\left(u_{1}+s, u_{2}, \ldots, u_{7}, u_{8}-s\right) e\left(\frac{\left(u_{8}-u_{1}-2 s\right)\left(u_{1}+u_{8}\right)}{2 \omega_{1} \omega_{2}}\right)
$$

under suitable parameter restraints by Proposition 4.8. By Theorem 6.1, we alternatively have

$$
\begin{aligned}
\lim _{s \rightarrow \infty} S_{h}\left(u_{1}+s, u_{2}, \ldots, u_{7}, u_{8}-s\right) e\left(\frac{\left(u_{8}-u_{1}-2 s\right)\left(u_{1}+u_{8}\right)}{2 \omega_{1} \omega_{2}}\right) \\
=\lim _{s \rightarrow \infty} \omega_{2} e\left(\frac{2 \omega^{2}+\sum_{j=2}^{5} u_{j}^{2}-u_{6}^{2}+u_{7}^{2}}{2 \omega_{1} \omega_{2}}\right) \frac{(\widetilde{q}, \widetilde{q} ; \widetilde{q})_{\infty}}{2} \frac{\theta\left(\widetilde{t}_{1} \widetilde{t}_{7} \widetilde{u} / \widetilde{q} ; \widetilde{q}\right) \prod_{j=2}^{5} \theta\left(\widetilde{t}_{j} \widetilde{t_{7}} / \widetilde{q} ; \widetilde{q}\right)}{\theta\left(\widetilde{t}_{7} / \widetilde{t}_{6} ; \widetilde{q}\right)} \\
\quad \times U_{t}\left(\widetilde{q}^{\frac{3}{2}} / \widetilde{t}_{7}, \widetilde{q}^{\frac{1}{2}} / \widetilde{t}_{2}, \ldots, \widetilde{q}^{\frac{1}{2}} / \widetilde{t_{6}}, \widetilde{q}^{\frac{1}{2}} / \widetilde{t}_{1} \widetilde{u}, \widetilde{q} \frac{3}{2} \widetilde{u} / \widetilde{t}_{8} ; \widetilde{q}\right) S_{t}\left(t_{6} / q, t_{2}, \ldots, t_{5}, t_{7}, t_{1} u, t_{8} / q u ; q\right) \\
\quad+\left(u_{6} \leftrightarrow u_{7}\right),
\end{aligned}
$$

where $u=e\left(i s / \omega_{2}\right)$ and $\tilde{u}=e\left(i s / \omega_{1}\right)$. We have $u, \widetilde{u} \rightarrow 0$ as $s \rightarrow \infty$ since $\Re\left(\omega_{1}\right), \Re\left(\omega_{2}\right)>0$, hence application of Proposition 5.18 gives the right-hand side of the desired identity with respect to the parameters $\left(u_{2}, \ldots, u_{7}\right)$.

Remark 6.4. Alternatively Corollary 6.3 can be proved by repeating the arguments of Theorem 6.1. The argument simplifies, since one now only needs the expression [5, (5.6.1)] of a very-well-poised ${ }_{8} \psi_{8}$ as a sum of two very-well-poised ${ }_{8} \phi_{7}$ series, and one does not need to use three term transformations for the trigonometric integrals.

We conclude this section by relating the $R$-function to the Askey-Wilson function using Corollary 6.3. The answer deviates from Ruijsenaars' $[27, \S 6.6]$ hunch that $R$ is (up to an elliptic prefactor) the product of an Askey-Wilson function in base $q$ and an Askey-Wilson function in base $\widetilde{q}$ : it is the appearance below of two such terms which upgrades the $W\left(D_{3}\right)$-symmetry of the Askey-Wilson functions to the $W\left(D_{4}\right)$-symmetry of $R$ (cf. Remark 6.2i)). For notational convenience, we write $w_{0}=-1 \in W\left(D_{4}\right)$ for 
the longest Weyl group element, acting as $w_{0} \gamma=-\gamma$ on the Askey-Wilson parameters $\gamma$. We define $\psi(\gamma ; x, \lambda)=\psi\left(\gamma ; x, \lambda ; \omega_{1}, \omega_{2}\right)$ by

$$
\begin{aligned}
\psi\left(\gamma ; x, \lambda ; \omega_{1}, \omega_{2}\right) & =\frac{h\left(\hat{\gamma} ; \lambda, x ; \omega_{1}, \omega_{2}\right)}{h\left(-\hat{\gamma} ; \lambda, x ; \omega_{1}, \omega_{2}\right)}\left(w_{0} \cdot \phi\right)\left(\gamma ; x, \lambda ; \omega_{1}, \omega_{2}\right) \\
& =\frac{h\left(\gamma ; x, \lambda ; \omega_{1}, \omega_{2}\right) h\left(\hat{\gamma} ; \lambda, x ; \omega_{1}, \omega_{2}\right)}{h\left(-\gamma ; x, \lambda ; \omega_{1}, \omega_{2}\right) h\left(-\hat{\gamma} ; \lambda, x ; \omega_{1}, \omega_{2}\right)} \phi\left(-\gamma ; x, \lambda ; \omega_{1}, \omega_{2}\right),
\end{aligned}
$$

where the gauge factor $h$ is given by (5.26). Note that $\psi\left(\gamma ; x, \lambda ; \omega_{1}, \omega_{2}\right)$ is a self-dual solution of the Askey-Wilson difference equation (5.23), see Remark 5.30. We furthermore define the multiplier

$$
M(\gamma ; x)=\frac{\theta\left(e\left(\left(\hat{\gamma}_{0}-\gamma_{3}-i x\right) / \omega_{2}\right), e\left(\left(\omega+\gamma_{2}+i x\right) / \omega_{2}\right), e\left(\left(\omega+\gamma_{3}-i x\right) / \omega_{2}\right) ; q\right)}{\theta\left(e\left(\left(\gamma_{3}-\hat{\gamma}_{0}-i x\right) / \omega_{2}\right), e\left(\left(\omega+\gamma_{0}-i x\right) / \omega_{2}\right), e\left(\left(\omega+\gamma_{1}-i x\right) / \omega_{2}\right) ; q\right)}
$$

which is elliptic in $x$ with respect to the period lattice $\mathbb{Z} i \omega_{1}+\mathbb{Z} i \omega_{2}$.

Theorem 6.5. We have

$$
\begin{aligned}
R\left(\gamma ; x, \lambda ; \omega_{1}, \omega_{2}\right)= & K(\gamma) M(\gamma ; x) M(\hat{\gamma} ; \lambda) \phi\left(s_{23} \gamma ; x, \lambda ; \omega_{1}, \omega_{2}\right) \\
& \times \psi\left(-\gamma ; x, \lambda ;-\omega_{2}, \omega_{1}\right)+\left(\gamma_{2} \leftrightarrow \gamma_{3}\right),
\end{aligned}
$$

where $s_{23} \gamma=\left(\gamma_{0}, \gamma_{1}, \gamma_{3}, \gamma_{2}\right)$ and with

$$
\begin{aligned}
& K\left(\gamma ; \omega_{1}, \omega_{2}\right) \\
&= \sqrt{-i} e\left(-\frac{1}{24}\left(\frac{\omega_{1}}{\omega_{2}}+\frac{\omega_{2}}{\omega_{1}}\right)\right) e\left(-\frac{3 \omega\left(\gamma_{0}+\gamma_{1}+\gamma_{2}-\gamma_{3}\right)}{2 \omega_{1} \omega_{2}}\right) \\
& \quad \times \frac{\prod_{j=1}^{3} G\left(i \omega+i \gamma_{0}+i \gamma_{j}\right)}{\theta\left(e\left(\left(\gamma_{2}-\gamma_{3}\right) / \omega_{2}\right) ; q\right)} e\left(\frac{-\gamma_{0}^{2}-\gamma_{1}^{2}-\gamma_{2}^{2}+\gamma_{3}^{2}-2 \gamma_{0} \gamma_{1}-2 \gamma_{0} \gamma_{2}+2 \gamma_{0} \gamma_{3}}{4 \omega_{1} \omega_{2}}\right) .
\end{aligned}
$$

Proof. Using the second hyperbolic Euler integral representation of $R$ from Theorem 4.21 and subsequently applying Corollary 6.3, we obtain an expression of $R\left(\gamma ; x, \lambda ; \omega_{1}, \omega_{2}\right)$ in terms of trigonometric integrals $E_{t}$ and $V_{t}$ with parameter specializations which allows us to rewrite them as Askey-Wilson functions by Lemma 5.31. This leads to the expression

$$
\begin{aligned}
R\left(\gamma ; x, \lambda ; \omega_{1}, \omega_{2}\right)= & C\left(\gamma ; x, \lambda ; \omega_{1}, \omega_{2}\right) \phi\left(s_{23} \gamma ; x, \lambda ; \omega_{1}, \omega_{2}\right) \psi\left(-\gamma ; x, \lambda ;-\omega_{2}, \omega_{1}\right) \\
& +\left(\gamma_{2} \leftrightarrow \gamma_{3}\right),
\end{aligned}
$$


with the explicit prefactor

$$
\begin{aligned}
& C\left(\gamma ; \lambda, x ; \omega_{1}, \omega_{2}\right) \\
& =\sqrt{\frac{\omega_{2}}{\omega_{1}}} e\left(\frac{8 \omega^{2}+8 x^{2}+2\left(i \omega-i \hat{\gamma}_{0}-\lambda\right)^{2}+\left(i \omega-2 i \gamma_{0}+i \hat{\gamma}_{0}+\lambda\right)^{2}}{8 \omega_{1} \omega_{2}}\right. \\
& \left.+\frac{\left(i \omega-2 i \gamma_{1}+i \hat{\gamma}_{0}+\lambda\right)^{2}-\left(i \omega-2 i \gamma_{2}+i \hat{\gamma}_{0}+\lambda\right)^{2}+\left(i \omega-2 i \gamma_{3}+i \hat{\gamma}_{0}+\lambda\right)^{2}}{8 \omega_{1} \omega_{2}}\right) \\
& \times \frac{(\widetilde{q} ; \widetilde{q})_{\infty}}{(q ; q)_{\infty}} \frac{\prod_{j=1}^{3} G\left(i \omega+i \gamma_{0}+i \gamma_{j}\right)}{\theta\left(e\left(\left(\gamma_{3}-\gamma_{2}\right) / \omega_{1}\right) ; \widetilde{q}\right)} \prod_{j=0}^{2} \frac{\left(e\left(\left(-\omega+\gamma_{j}+i x\right) / \omega_{1}\right) ; \widetilde{q}\right)_{\infty}}{\left(e\left(\left(-\omega-\gamma_{j}+i x\right) / \omega_{1}\right) ; \widetilde{q}\right)_{\infty}} \\
& \times \frac{\theta\left(e\left(\left(-\omega+\gamma_{3} \pm i x\right) / \omega_{1}\right) ; \tilde{q}\right)\left(e\left(\left(\omega-\gamma_{2} \pm i x\right) / \omega_{2}\right) ; q\right)_{\infty} \prod_{j=0}^{3} G\left(-i \gamma_{j} \pm x\right)}{\left(e\left(\left(\omega+\gamma_{0} \pm i x\right) / \omega_{2}\right), e\left(\left(\omega+\gamma_{1} \pm i x\right) / \omega_{2}\right), e\left(\left(\omega+\gamma_{3} \pm i x\right) / \omega_{2}\right) ; q\right)_{\infty}} \\
& \times \frac{\theta\left(e\left(\left(\gamma_{3}-\hat{\gamma}_{0}+i x\right) / \omega_{1}\right) ; \tilde{q}\right)}{\left.\theta\left(\left(\hat{\gamma}_{0}-\gamma_{3}+i x\right) / \omega_{1}\right) ; \tilde{q}\right)} \frac{\left(e\left(\left(-\omega+\gamma_{3}-i x\right) / \omega_{1}\right) ; \tilde{q}\right)_{\infty}}{\left(e\left(\left(-\omega-\gamma_{3}-i x\right) / \omega_{1}\right) ; q\right)_{\infty}} \\
& \times \frac{\theta\left(e\left(\left(\hat{\gamma}_{3}-\gamma_{0}+i \lambda\right) / \omega_{1}\right), e\left(\left(-\omega-\hat{\gamma}_{2}+i \lambda\right) / \omega_{1}\right), e\left(\left(-\omega+\hat{\gamma}_{3}+i \lambda\right) / \omega_{1}\right) ; \widetilde{q}\right)}{\theta\left(e\left(\left(\gamma_{0}-\hat{\gamma}_{3}+i \lambda\right) / \omega_{1}\right) ; \widetilde{q}\right)} \\
& \times \frac{G\left(\lambda-i \hat{\gamma}_{1}, \lambda-i \hat{\gamma}_{2}, \lambda-i \hat{\gamma}_{3}\right)}{G\left(\lambda+i \hat{\gamma}_{0}\right)} \\
& \times \frac{\left(e\left(\left(-\omega+\hat{\gamma}_{3}-i \lambda\right) / \omega_{1}\right) ; \widetilde{q}\right)_{\infty}}{\left(e\left(\left(-\omega-\hat{\gamma}_{3}-i \lambda\right) / \omega_{1}\right) ; \widetilde{q}\right)_{\infty}} \prod_{j=0}^{2} \frac{\left(e\left(\left(-\omega+\hat{\gamma}_{j}+i \lambda\right) / \omega_{1}\right) ; \widetilde{q}\right)_{\infty}}{\left(e\left(\left(-\omega-\hat{\gamma}_{j}+i \lambda\right) / \omega_{1}\right) ; \widetilde{q}\right)_{\infty}} \\
& \times \frac{\left(e\left(\left(-\omega-\hat{\gamma}_{3}+i \lambda\right) / \omega_{1}\right) ; \widetilde{q}\right)_{\infty}}{\left(e\left(\left(-\omega+\hat{\gamma}_{0}+i \lambda\right) / \omega_{1}\right), e\left(\left(-\omega+\hat{\gamma}_{1}-i \lambda\right) / \omega_{1}\right), e\left(\left(-\omega+\hat{\gamma}_{2}-i \lambda\right) / \omega_{1}\right) ; \widetilde{q}\right)_{\infty}} \\
& \times \frac{\left(e\left(\left(\omega-\hat{\gamma}_{2}-i \lambda\right) / \omega_{2}\right) ; q\right)_{\infty}}{\left(e\left(\left(\omega+\hat{\gamma}_{0}-i \lambda\right) / \omega_{2}\right), e\left(\left(\omega+\hat{\gamma}_{1}+i \lambda\right) / \omega_{2}\right), e\left(\left(\omega+\hat{\gamma}_{3}+i \lambda\right) / \omega_{2}\right) ; q\right)_{\infty}} .
\end{aligned}
$$

Elaborate but straightforward computations using (6.1), (6.4) and (6.5) now yields the desired result.

Acknowledgements. Rains was supported in part by NSF Grant No. DMS-0401387. Stokman was supported by the Netherlands Organization for Scientific Research (NWO) in the VIDI-project "Symmetry and modularity in exactly solvable models".

\section{References}

1. van de Bult, F.J.: Ruijsenaars' hypergeometric function and the modular double of $\mathcal{U}_{q}\left(\mathfrak{s} \mathfrak{L}_{2}(\mathbb{C})\right)$. Adv. Math. 204(2), 539-571 (2006)

2. van Diejen, J.F.: Integrability of difference Calogero-Moser systems. J. Math. Phys. 35(6), 29833004 (1994)

3. Faddeev, L.: Modular double of a Quantum Group. In: “Conférence Moshé Flato” 1999, Vol I (Dijon), Math. Phys. Stud. 21, Dordrecht: Kluwer Acad. Publ., 2000, pp. 149-156 
4. Frenkel, I.N., Turaev, V.G.: Elliptic solutions of the Yang-Baxter equation and modular hypergeometric functions. In: Arnold, V.I., Gelfand, I.M., Retakh, V.S., Smirnov, M. (eds.) The Arnold-Gelfand mathematical seminars., pp. 171-204. Birkhäuser, Boston, 1997

5. Gasper, G., Rahman, M.: Basic Hypergeometric Series, Encyclopedia of Mathematics and its Applications, Vol. 96, 2nd ed., Cambridge Univ. Press, Cambridge, 2004

6. Groenevelt, W.: The Wilson function transform. Int. Math. Res. Not. 2003(52), 2779-2817 (2003)

7. Gupta, D.P., Masson, D.R.: Contiguous relations, continued fractions and orthogonality. Trans. Amer. Math. Soc. 350(2), 769-808 (1998)

8. Heckman, G., Schlichtkrull, H.: Harmonic Analysis and Special Functions on Symmetric Spaces. Perspectives in Mathematics, Vol. 16, 1994

9. Ismail, M.E.H., Rahman, M.: Associated Askey-Wilson polynomials. Trans. Amer. Math. Soc. 328, 201-239 (1991)

10. Koelink, E., Stokman, J.V.: Fourier transforms on the quantum SU(1, 1) quantum group (with an appendix of M. Rahman). Publ. Res. Math. Sci. 37(4), 621-715 (2001)

11. Koelink, E., Stokman, J.V.: The Askey-Wilson function transform. Int. Math. Res. Not. 2001(22), 1203-1227 (2001)

12. Koelink, E., van Norden, Y., Rosengren, H.: Elliptic U(2) quantum group and elliptic hypergeometric series. Commun Math. Phys 245(3), 519-537 (2004)

13. Komori, Y., Hikami, K.: Quantum integrability of the generalized elliptic Ruijsenaars models. J. Phys. A: Math. Gen. 30, 4341-4364 (1997)

14. Letzter, G.: Quantum zonal spherical functions and Macdonald polynomials. Adv. Math. 189(1), 88147 (2004)

15. Lievens, S., Van der Jeugt, J.: Symmetry groups of Bailey's transformations for ${ }_{10} \phi_{9}$-series. J. Comput. Appl. Math. 206(1), 498-519 (2007)

16. Macdonald, I.G.: Orthogonal polynomials associated to root systems. Sém. Lothar. Combin. 45, Art. B45a (2000/01)

17. Nassrallah, B., Rahman, M.: Projection formulas, a reproducing kernel and a generating function for $q$-Wilson polynomials. SIAM J. Math. Anal. 16, 186-197 (1985)

18. Noumi, M.: Macdonald's symmetric polynomials and as zonal spherical functions on some quantum homogeneous spaces. Adv. Math. 123(1), 16-77 (1996)

19. van der Put, M., Singer, M.F.: Galois theory of difference equations. Lecture Notes in Mathematics 1666. Springer-Verlag, Berlin, 1997

20. Rahman, M.: An integral representation of a ${ }_{10} \phi 9$ and continuous biorthogonal $10 \phi_{9}$ rational functions. Canad. J. Math. 38, 605-618 (1986)

21. Rains, E.M.: Tranformations of elliptic hypergeometric integrals. Ann. Math., to appear 2008

22. Rains, E.M.: Recurrences for elliptic hypergeometric integrals in elliptic integrable systems. In: Noumi, M., Takasaki, K. (eds.) Rokko Lectures in Mathematics 18, pp. 183-199, Kobe, Japan (2005)

23. Rains, E.M.: Limits of elliptic hypergeometric integrals. http://arxiv.org/list/math.CA/0607093, 2006

24. Ruijsenaars, S.N.M.: First order analytic difference equations and integrable quantum systems. J. Math. Phys. 38, 1069-1146 (1997)

25. Ruijsenaars, S.N.M.: Systems of Calogero-Moser type. In: Particles and Fields (Banff, AB, 1994), CRM Ser. Math. Phys., New York: Springer, 1999, pp. 251-352

26. Ruijsenaars, S.N.M.: A generalized hypergeometric function satisfying four analytic difference equations of Askey-Wilson type. Commun Math. Phys. 206(3), 639-690 (1999)

27. Ruijsenaars, S.N.M.: Special functions defined by analytic difference equations. In: Special functions 2000: Current perspective and future directions (Tempe, AZ), NATO Sci. Ser. II Math. Phys. Chem. 30, Dordrecht: Kluwer Acad. Publ., 2001, pp. 281-333

28. Ruijsenaars, S.N.M.: A generalized hypergeometric function II. Asymptotics and $D_{4}$ symmetry. Comm. Math. Phys. 244(3), 389-412 (2003)

29. Ruijsenaars, S.N.M.: A generalized hypergeometric function III. Associated Hilbert space transform. Commun. Math. Phys. 243(3), 413-448 (2003)

30. Shintani, T.: On a Kronecker limit formula for real quadratic fields. J. Fac. Sci. Univ. Tokyo, Sect. 1A 24, 167-199 (1977)

31. Spiridonov, V.P.: On the elliptic beta function. Russ. Math. Surv. 56(1), 185-186 (2001)

32. Spiridonov, V.P.: Short proofs of the elliptic beta integrals. Ramanujan J. 13, 265-283 (2007)

33. Spiridonov, V.P.: Classical elliptic hypergeometric functions and their applications. Rokko Lect. in Math. Vol. 18, Dept. of Mth., Kobe Univ., 2005, pp. 253-287

34. Spiridonov, V.P.: Theta hypergeometric integrals. Algebra i Analiz 15, 161-215 (2003) (St. Petersburg Math J. 15, 929-967) (2004)

35. Spiridonov, V.P., Zhedanov, A.S.: Spectral transformation chains and some new biorthogonal rational functions. Commun Math. Phys. 210, 49-83 (2000) 
36. Stokman, J.V.: Difference Fourier transforms for nonreduced root systems. Selecta Math. (N.S.) 9(3), 409494 (2003)

37. Stokman, J.V.: Hyperbolic beta integrals. Adv. Math. 190, 119-160 (2005)

Communicated by L. Takhtajan 